\title{
Experimental Investigation of Aerodynamic Devices for Wind Turbine Rotational Speed Control: Phase II
}

L. Scott Miller, Ph. D.

Wichita State University

Wichita, Kansas

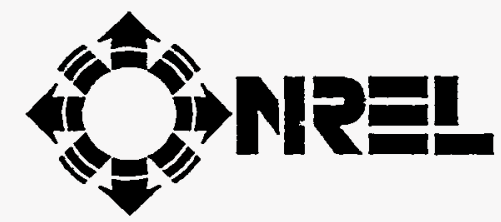

National Renewable Energy Laboratory 1617 Cole Boulevard Golden, Colorado 80401-3393

A national laboratory of the U.S. Department of Energy Managed by Midwest Research Institute for the U.S. Department of Energy under Contract No. DE-AC36-83CH10093 



\section{Experimental Investigation of Aerodynamic Dexices for Wind Turbine Rotatonal Speed Control: Phase II}

L. Scott Miller, Ph. D.

Wichita State University

Wichita, Kansas

NREL Technical Monitor:

Paul Migliore
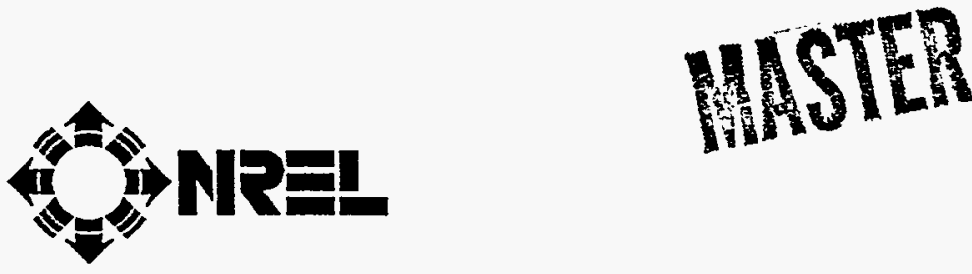

National Renewable Energy Laboratory 1617 Cole Boulevard Golden, Colorado 80401-3393

A national laboratory of the U.S. Department of Energy Managed by Midwest Research Institute for the U.S. Department of Energy under contract No. DE-AC36-83CH10093

Prepared under Subcontract No. XF-1-11009-3

February 1996

DISTRIBUTION OF THIS DOCUMENT IS UNLIMITED 35 


\section{NOTICE}

This report was prepared as an account of work sponsored by an agency of the United States government. Neither the United States government nor any agency thereof, nor any of their employees, makes any warranty, express or implied, or assumes any legal liability or responsibility for the accuracy, completeness, or usefulness of any information, apparatus, product, or process disclosed, or represents that its use would not infringe privately owned rights. Reference herein to any specific commercial product, process, or service by trade name, trademark, manufacturer, or otherwise does not necessarily constitute or imply its endorsement, recommendation, or favoring by the United States govemment or any agency thereof. The views and opinions of authors expressed herein do not necessarily state or reflect those of the United States government or any agency thereof.

Available to DOE and DOE contractors from:

Office of Scientific and Technical Information (OSTI)

P.O. Box 62

Oak Ridge, TN 37831

Prices available by calling (423) $576-8401$

Available to the public from:

National Technical Information Service (NTIS)

U.S. Department of Commerce

5285 Port Royal Road

Springfield, VA 22161

(703) $487-4650$ 


\section{Foreword}

The Wind Technology Division of the National Renewable Energy Laboratory (NREL) is conducting exploratory research on aerodynamic devices that are intended to enhance wind-turbine rotor performance and attenuate structural loads. Desired properties of these devices include simplicity, reliability, maintainability, low cost and fail-safe design. Initial efforts have focused on the use of trailing-edge aerodynamic brakes for overspeed protection. Long-term efforts will address more aggressive and innovative strategies that have the potential to significantly advance the state of the art.

This report touches on the work performed in two projects: Subcontract No. TAD-3-13400 entitled "Wind Turbine Trailing-Edge Aerodynamic Brake Design" performed by Gene A. Quandt, and Subcontract No. XAD-3-133365 entitled "Aerodynamic Devices for Wind Turbine Performance Enhancement" performed by Wichita State University (WSU). These two projects progressed in parallel, with considerable interaction between the principal investigators.

The WSU Phase-1 Report discussed the configurations studied and the attempts to identify promising alternatives through the analysis of the wind tunnel test data. This Phase-2 Report presents windtunnel results for "spoiler-flaps" of $30 \%, 40 \%$ and $50 \%$ chord; for various leading-edge lip extensions; for different venting arrangements; and for different device hinge locations. Gene Quandt's subcontract report, No. TP-441-7389, focuses on aerodynamic and structural design, and includes preliminary design calculations for a centrifugally-actuated aerodynamic brake.

As is often the case with exploratory research, these projects have spawned additional follow-on studies. Wind-tunnel tests are planned at Ohio State University (OSU) in which a pressure-tapped S809 airfoil model will be tested with three trailing-edge devices: the spoiler-flap, a plain flap ("unvented aileron") and a vented plain flap ("vented aileron"). Rotating-blade tests of these same configurations will be conducted at the National Wind Technology Center (NWTC), with the goal of quantifying the effects of unsteadiness, blade rotation and aspect ratio, so that corrections can be applied to wind-tunnel test data for use by wind-turbine designers in the future.

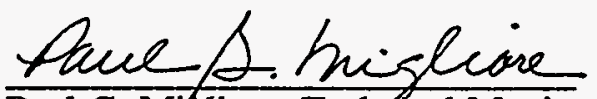

Paul G. Migliore, Techucal Monitor 


\section{Preface}

The present work describes the second phase of research intended to identify improved aerodynamic devices for wind turbine overspeed protection and power modulation applications. On the basis of results obtained in a previous investigation, the trailing-edge device, known as the Spoiler-Flap, was examined further during wind tunnel tests. The impact of lip length, vent angle, pivot point and chord variations on aerodynamic and hinge moment characteristics were evaluated and a best overall configuration was identified.

As was the case in the Phase 1 activities, this work is the product of a group effort. Paul Migliore, of the National Renewable Energy Laboratory (NREL), and Gene Quandt, a consultant, deserve a great deal of credit. Paul provided exceptional guidance, advice and help during the course of the project. Gene, once again, proved most skilled at identifying aerodynamic devices that will potentially offer benefits to the wind industry for years to come. He is a master engineer and he deserves recognition for his work.

A number of people at WSU participated in this project. Without their skills and expertise the work could not have been completed. Art Porter, of the engineering shop, again produced a wind tunnel model of superior quality. His design and manufacturing skills are unsurpassed and he is truly a craftsman and engineer second to none. Bonnie Johnson, of the WSU Walter H. Beech Memorial 7 X 10 foot low speed wind tunnel, and all her student workers (Jennifer Reiley, Mark Smaglik, Greg Thumman and Tram Vu) again provided outstanding help and data. It is a pleasure to work in the tunnel. Last, but certainly not least, I want to recognize two of my graduate students. Steve Huang and Ihssane Mounir spent many long hours working on this project. They did an incredible job getting ready for the tests, working in the tunnel and analyzing the results. I know they learned a lot and I hope they had a good time.

To properly educate future engineers, it is necessary to expose students to the problems, needs and capabilities of industry and research laboratories. Traditional classroom educational experiences are necessary, but additional "hands-on" exposure to the world of engineering is often more valuable. Unfortunately, it can be difficult to provide such a learning experience to students. Funding of projects, such as this one, provide an outstanding opportunity to enhance the quality of students in an extremely efficient and effective way. . The National Renewable Energy Laboratory (NREL), the US Department of Energy (DOE), and all involved are to be commended for their support. Their educational contributions are positive and immeasurable. 


\section{Summary}

An experimental investigation was undertaken to further evaluate and enhance the performance of an aerodynamic device for wind turbine overspeed protection and power modulation applications. The trailing-edge device, known as the Spoiler-Flap; was examined in detail during wind tunnel tests. The impact of lip length, vent angle, pivot point and chord variations on aerodynamic and hinge moment characteristics were evaluated and a best overall configuration was identified. Based on this effort, a $40 \%$ chord device with a $1 \%$ lip length and 40 degree vent angle offers improved performance potential for wind turbine applications. This specific configuration appears to offer good suction coefficient performance for both turbine power modulation and overspeed (i.e., aerodynamic braking) applications. Device hinge moment loads improved (compared to other devices investigated) in magnitude and the impact of surface roughness was found to be minimal. 


\section{Contents}

Nomenclature $\quad 1$

Introduction $\quad 2$

Spoiler-Flap Test Configurations 3

Wind Tunnel Investigation $\quad 7$

$\begin{array}{ll}\text { Aerodynamic Coefficient Results and Discussion } & 10\end{array}$

$\begin{array}{ll}\text { Hinge Moments } & 48\end{array}$

Best Device Configuration

$\begin{array}{ll}\text { Conclusions } & 69\end{array}$

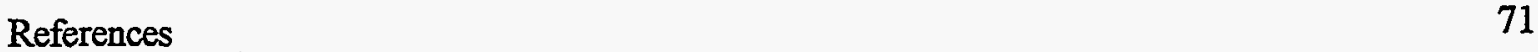




\section{Nomenclature}

c Airfoil chord, including the device

c Device chord

L Section lift, per unit span, acting perpendicular to approach flow direction

D Section drag, per unit span, acting parallel to approach flow direction

$M_{c} \quad$ Device hinge moment, per unit span; trailing-edge down is positive

$\mathrm{C}_{\mathrm{I}} \quad$ Section lift coefficient, $(\mathrm{L}) /[(\mathrm{q})(\mathrm{c})]$

$C_{d} \quad$ Section drag coefficient, $(D) /[(q)(c)]$

$\mathrm{C}_{\mathrm{q}}$ Total turbine rotor torque coefficient, $(\mathrm{Q}) /\left[1 / 2(\rho)\left(\Pi \mathrm{R}^{2}\right)(\Omega \mathrm{R})^{2}(\mathrm{R})\right]$

$\mathrm{C}_{\mathrm{s}} \quad$ Section suction coefficient, $\left(\mathrm{C}_{\mathrm{l}}\right)(\sin \alpha)-\left(\mathrm{C}_{\mathrm{d}}\right)(\cos \alpha)$

$\mathrm{Cm}_{\mathrm{h}} \quad$ Device hinge moment coefficient, $\left(\mathrm{M}_{\mathrm{c}}\right) /\left[(\mathrm{q})\left(\mathrm{c}_{\mathrm{c}}{ }^{2}\right)\right]$

Q Total turbine rotor torque

q Dynamic pressure, $(0.5)(\rho)\left(V^{2}\right)$

$\mathrm{R} \quad$ Rotor radius

$\mathrm{R}_{\mathrm{c}} \quad$ Reynolds number, $[(\mathrm{V})(\rho)(\mathrm{c})] /(\mu)$

V Relative inflow velocity

$\mathrm{V}_{\mathrm{w}} \quad$ Wind velocity

X Tip-speed-ratio, $(\Omega R) / V_{W}$

a Airfoil angle of attack; nose up is positive

$\delta \quad$ Trailing-edge device deflection angle; trailing edge down is positive

$\mu \quad$ Absolute viscosity of air

$\eta \quad$ Normalized device effectiveness

$\Omega \quad$ Rotor rotational speed

$\rho \quad$ Mass density of air 


\section{Introduction}

In light of the need to produce energy using more robust, reliable and efficient wind turbine designs, a range of new technologies has been explored. Trailing-edge aerodynamic devices represent one such technology that offers the potential for improved horizontal axis wind turbine performance. Indeed, these devices are particularly attractive for two reasons: to prevent turbine rotation speeds from exceeding reasonable limits during high wind or loss of load situations, and for power modulation.

Results from a previous investigation (Miller, 1995) identified and evaluated the capabilities of five trailing-edge aerodynamic devices on an NACA $64_{3}-618$ airfoil. The primary focus of the Phase 1 work was to enhance braking capabilities for wind turbines during overspeed situations. Power modulation aspects were not specifically addressed.

The aerodynamic capability of each Phase 1 device configuration was evaluated during low speed wind tunnel tests at Wichita State University (WSU). Besides comparing and contrasting the aerodynamic behavior of each device, data from these tests were also input into a turbine performance computer program to evaluate their potential for overspeed protection on a generic wind turbine design. Based on these Phase 1 results, a particular device configuration was singled out for further investigation and enhancement. The Spoiler-Flap trailing-edge aerodynamic device, as shown in Figure 1, appeared particularly promising.

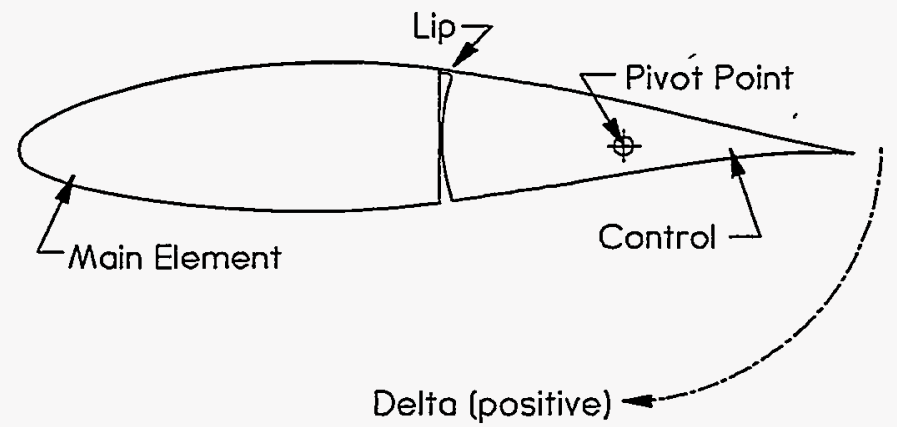

Figure 1. A diagram of the Spoiler-Flap device configuration on an NACA $643^{-618}$ airfoil, as defined in Phase 1

Phase 2 efforts, involving additional wind tunnel testing, were performed in an attempt to improve or optimize this particular configuration's performance and applicability for use on wind turbines. The impact of lip length, vent angle, pivot location and device chord were examined further. A significant amount of experimental data was gathered on lift, drag, quarter chord moment, and device hinge moment coefficients as a function of angle of attack and device deflection. This report documents the results of the Phase 2 investigation. 


\section{Spoiler-Flap Test Configurations}

A number of geometry variations were examined in an attempt to identify an enhanced Spoiler-Flap configuration that offers improved performance and characteristics suited to wide use on typical wind turbine designs.

Tests centered on evaluating the effect of lip length, vent angle, and pivot location on device performance. Initial measurements focused on the $50 \%$ chord device. Results from these tests were utilized to identify general trends and thus to reduce the range of permutations examined for other, shorter, chord devices. As a consequence, the number of lip length, vent angle, and pivot location variations studied for the $40 \%$ and $30 \%$ chord devices was greatly reduced.

It is important to note that all device chords and lip lengths are expressed, throughout this report, in terms of a percentage of an 18.0 inch chord airfoil. However, the last 0.75 inches of the airfoil was cut off during manufacturing to assure that a reasonable trailing-edge thickness resulted.

The base airfoil shape for the model was approximately that of an 18.0 inch chord NACA $64_{3}-618$ section, with a 0.75 inch trailing-edge truncation. All of the devices are assumed to match this shape when in an undeployed condition. However, once the devices are actuated the airfoil geometry changes notably. The vent angle is defined as the device deflection angle (i.e., the delta or $\delta$ value) above which free or unimpeded airflow between the airfoil upper and lower surfaces can occur. A basic description and schematic diagram for each configuration examined are provided in the following sections, and specifically in Figures 2-8. The four circles superimposed or drawn near the trailing edge represent a possible pivot point for each device. Specific information on the pivot points examined follows in a related section.

\section{0\% Chord Devices}

Figures 2-5 show schematic diagrams of the $50 \%$ chord Spoiler-Flap configurations examined. The cove region geometry necessary to change the vent angle are clearly identified in these figures. Lip length variations are not shown.

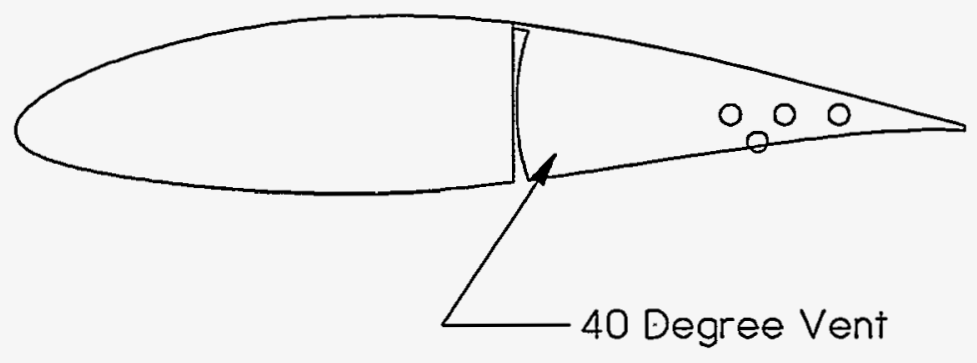

Figure 2. 50\% chord device, with a $1 \%$ lip length and 40 degree vent configuration 


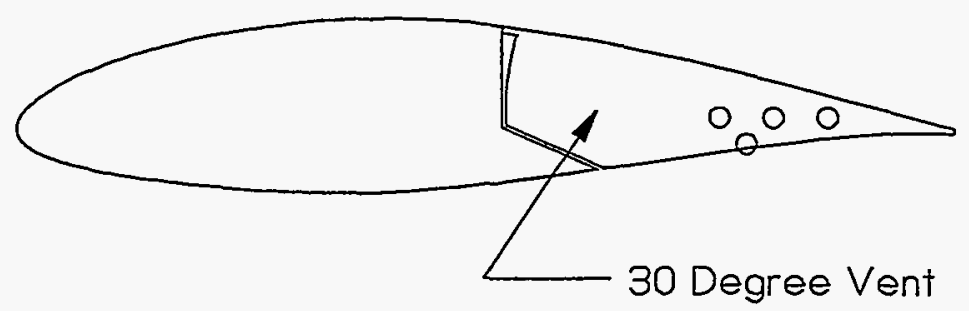

Figure $3.50 \%$ chord device, with a $1 \%$ lip length and 30 degree vent configuration

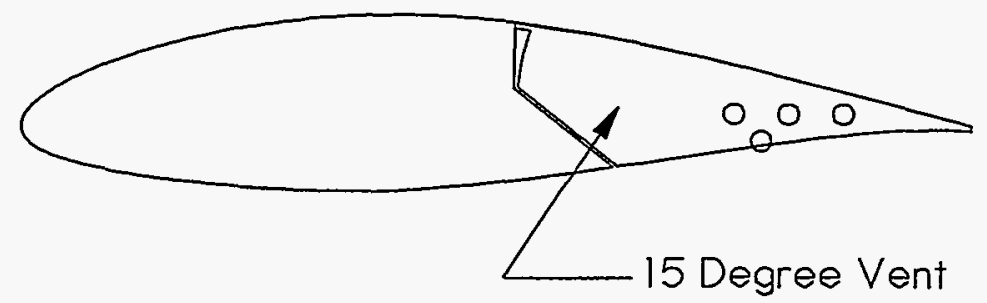

Figure 4. 50\% chord device, with a $1 \%$ lip length and 15 degree vent configuration

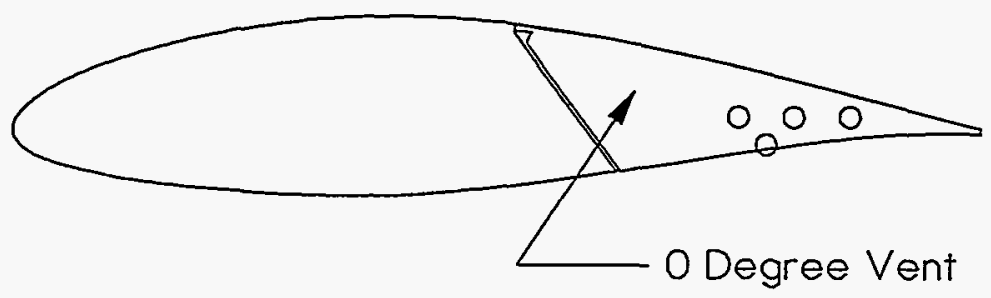

Figure 5. 50\% chord device, with a $1 \%$ lip length and 0 degree vent configuration

\section{0\% Chord Devices}

Figures 6 and 7 are schematic diagrams of the $40 \%$ chord Spoiler-Flap configurations examined. Again, the cove region geometry necessary to control the vent angle are clearly identified in these figures. A fixed lip length of approximately $1 \%$ is shown.

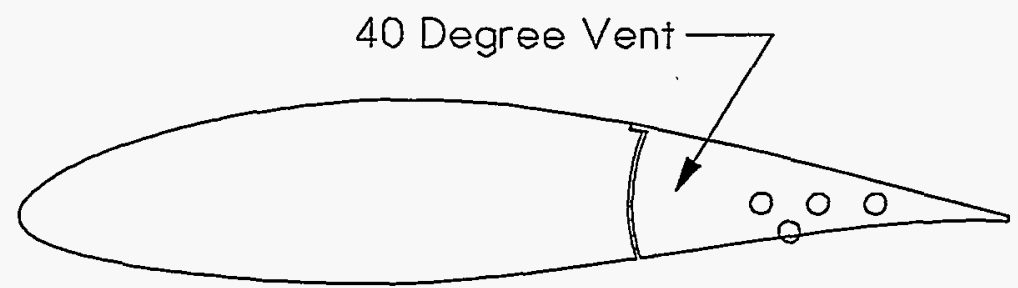

Figure 6. $40 \%$ chord device, with a $1 \%$ lip length and 40 degree vent configuration 


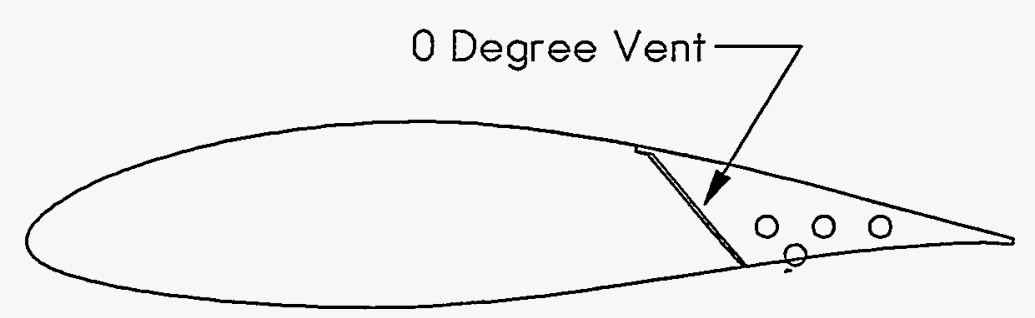

Figure 7. $40 \%$ chord device, with a $1 \%$ lip length and 0 degree vent configuration

\section{$30 \%$ Chord Devices}

Figure 8 shows the $30 \%$ chord Spoiler-Flap configuration. The pivot location and cove region geometry is clearly identified in this figure; however, lip length variations are not shown.

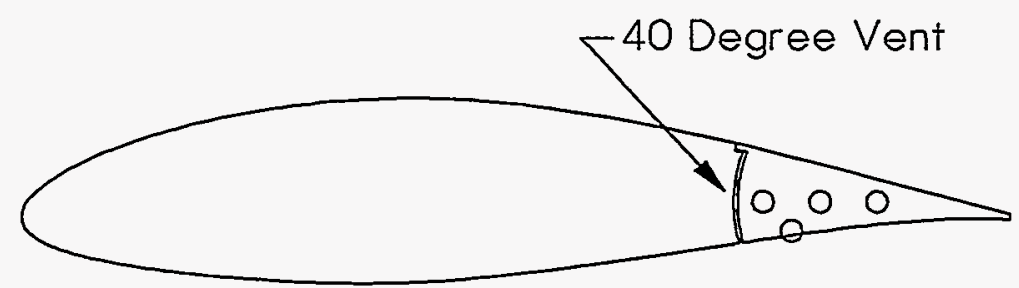

Figure $8.30 \%$ chord device, with a $1 \%$ lip length and 40 degree vent configuration

\section{Pivot Point Locations}

Figure 9 shows the numbering system used to identify each pivot point location. Unless indicated otherwise, the $50 \%$ chord device used pivot 3 , the $40 \%$ chord device used pivot 4 , and the $30 \%$ chord device used pivot 5 . Pivots 3,4 , and 5 were located approximately $1 \%$ above the airfoil chord line and, respectively, at the $75 \%, 80 \%$, and $85 \%$ chord locations. Pivot 6 was about $2.5 \%$ below the chord line and at the $77.5 \%$ chord location.

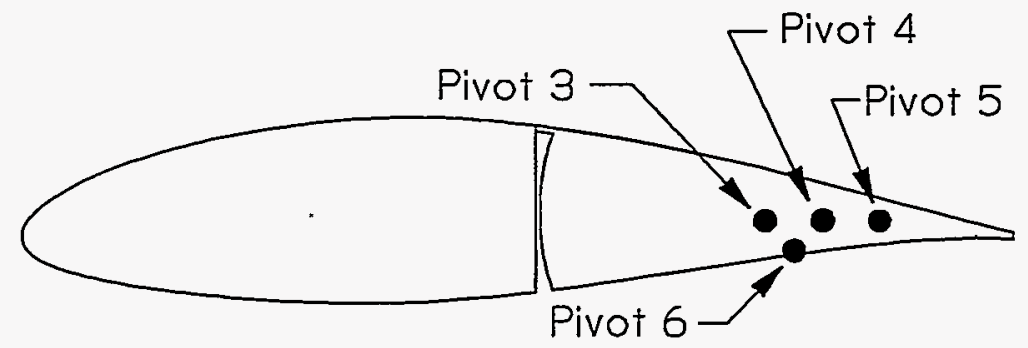

Figure 9. A diagram labeling the device pivot points (a 50\% chord device, with a $1 \%$ lip and 40 degree vent angle is shown)

\section{Best Device Configuration}

As the -wind tunnel tests progressed, a final "best" device configuration was identified. The order of presentation of results, in the next section, is approximately that of the investigation. The process of 
selecting the "best" configuration is thus roughly illustrated. The exact geometry or configuration of this device is presented in the results section.

Additional testing was performed to further evaluate the best device's performance. Specifically, a wider range of device deflections were examined and roughness was applied to the leading edges in an attempt to assess power modulation and insect accumulation or blade surface damage effects. A gurney flap was also installed, on the device's lower-surface trailing edge, in order to evaluate possible further performance improvements. 


\section{Wind Tunnel Investigation}

Each of the configurations described above was evaluated in the WSU Walter Beech Memorial 7 X 10 foot low-speed wind tunnel (Johnson, 1993). This particular facility is of a closed-return design and is capable of test section dynamic pressures up to 60 pounds per square foot (psf). The aerodynamic performance of the devices was evaluated in detail during approximately 40 hours of tests. The following sections discuss, in detail, specific aspects related to the design, installation, and testing of the model configurations.

The wind tunnel model utilized for the Phase 1 work was again used, with necessary trailing-edge device changes, to complete the Phase 2 activities. Important information related to the model features and wind tunnel testing procedures will be briefly summarized in the following. More detailed information can be found in the Phase 1 report (Miller, 1995). Unless otherwise noted, the previously supplied model descriptions and wind tunnel testing methods apply.

\section{Wind Tunnel Model Installation}

The model was mounted in the test section vertically from the tunnel floor to the ceiling, thus forming a two-dimensional installation. Circular end plates, with a diameter of 27-inches, were attached to the model and served to minimize three-dimensional and leakage flow near the tunnel walls. The end plates were approximately 0.187 -inches-thick and had smoothly rounded edges.

The model was supported by two wind tunnel balances that together measured the net two-dimensional lift, drag, and quarter-chord pitching moments loads. A six-component pyramidal type external balance recorded approximately half of the model lift and drag forces and all of the pitching-moments about the quarter-chord. A specially constructed two-component balance, connected to the top end of the model, measured the remaining model lift and drag. The output from the two balances was combined to determine total model aerodynamic loads.

\section{Model Details}

A wind tunnel model, originally supplied courtesy of Northern Power Systems (NPS), was modified in the WSU machine shop to serve as the basic test apparatus. The main element, or forward part, of the basic model, which has an approximately 9.0-inch chord and 84-inch span, was used during the entire investigation. New or modified parts were installed to the aft half of the model as necessary to produce the various test configurations.

All of the aerodynamic configurations examined required device deflections through a range of different deflection angles (i.e., delta or $\delta$ values). An electrically controlled actuator, positioned below the tunnel floor, was used for this purpose. A rotating drive shaft, actuating arm and push rod were the only parts of the actuator mechanism that were exposed to the flow during the tests. Figure 10 shows an end view of the model and device deflection actuator system. The actuating arm and push rod were approximately $0.75-$ inches high and wide.

During Phase 1 , device hinge moments were calculated using static pressure distributions about the surface. The accuracy of the moment data acquired in this fashion is thus highly dependent on the total number and location of pressure taps available. To measure hinge moments during the Phase 2 investigation, both the actuating arm and push rod were fit with strain gages. This technique is more direct and requires less post-investigation data analysis. Prior to testing, a full hinge moment instrumentation calibration was performed using a weight and pulley system. Output from the gages was acquired and recorded using the wind tunnel data system. 


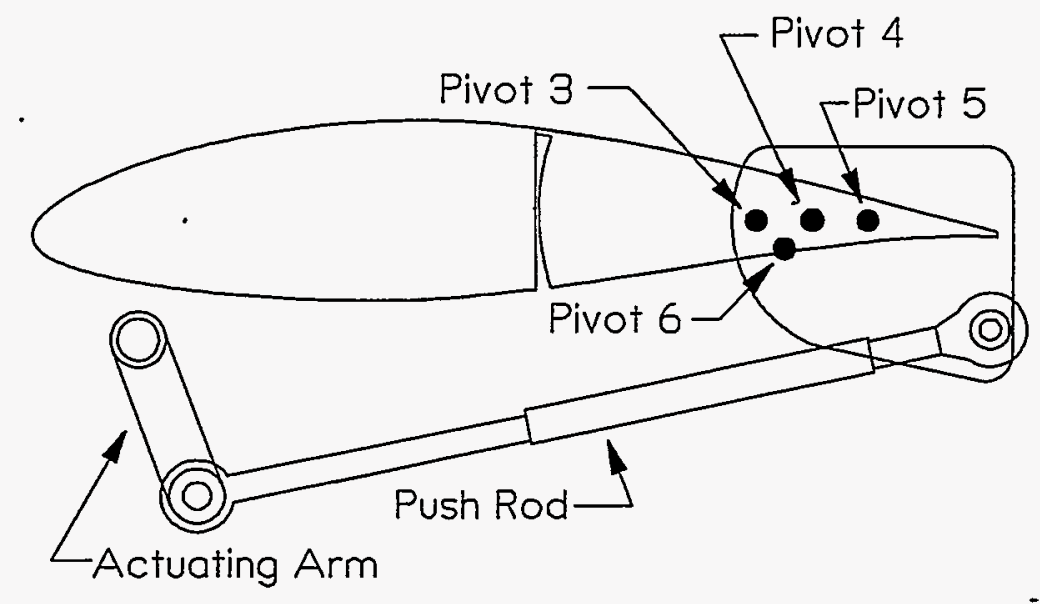

Figure 10. A schematic diagram showing the wind tunnel model device actuation mechanism, on a $\mathbf{5 0 \%}$ chord device

\section{Wind Tunnel Data Acquisition}

Data from the various measurement sensors used during the tests were acquired and conditioned as necessary by the wind tunnel data acquisition system. The WSU facility utilizes a HP-9000 series workstation computer to control and gather test results. This computer is connected to a stand-alone unit, an HP-3852, that provides signal conditioning for various types of measurement instruments and a buffer interface with the host computer. Model loads, model angle of attack, tunnel dynamic pressure, and test ${ }^{-}$ section temperature are continuously monitored and recorded in order to allow accurate measurement of a model's aerodynamic character. Appropriate data reduction methods are applied to the measurements as needed, and the resulting data is presented in a real-time fashion, as well as in hard-copy or software formats (Johnson, 1993).

\section{Wind Tunnel Blockage and Flow Corrections}

Lift, drag and pitching moment data measured directly by the tunnel balances were corrected to account for effects related to testing in the unique environment of a wind tunnel. Standard two-dimensional methods, as presented in Rae and Pope, were utilized to correct the test data for the influence of flow blockage (solid and wake), flow angularity, streamline curvature, and buoyancy (Rae \& Pope, 1984).

Model tare and interference drag effects were not measured or corrected during the current investigation. However, it is assumed that the wake-momentum-based values obtained during the Phase 1 work would apply to the current results. Previous tare and interference drag coefficients were observed to be approximately constant and equal to 0.020 , for the observed angle of attack range.

\section{Wind Tunnel Test Conditions}

Basic two-dimensional force and moment data were recorded for each test configuration over an angle of attack range between -6 and +90 degrees, and for device deflections from 0 to +90 degrees. In addition, device hinge moment measurements were also performed. Tunnel operating dynamic pressures were set as high as feasible (about 15 pounds per square foot or $80 \mathrm{mph}$ ) to provide the largest Reynolds number data possible $(\sim 1,000,000$ at the highest speed, based on full chord). 
Interestingly, model buffet levels limited wind tunnel test speeds more than any other factor. Tunnel balance load limits were rarely approached. Typically, the models remained stable and steady as angle of attack increased until about 25 degrees, at which time buffet would start. As angle of attack or device deflection increased, the tunnel operator was typically forced to reduce the dynamic pressure (or wind speed). Under the worst conditions, the tunnel dynamic pressure would be lowered to a value of 3.0 or 5.0 psf by the time tests reached 90 degrees angle of attack. 


\section{Aerodynamic Coefficient Results and Discussion}

Traditionally, when one evaluates the aerodynamic performance of an airfoil, the lift and drag behavior receive the greatest scrutiny. However, the suction coefficient behavior is of primary interest in the current investigation and it will receive the most attention during the results discussion. It is the magnitude and sign of this term that suggests the device's potential for turbine overspeed control or power modulation applications.

The following brief discussion justifies the relevant interest in the suction coefficient $\left(C_{S}\right)$ behavior. Considering only the aerodynamic behavior of the airfoil device, $\mathrm{C}_{\mathrm{s}}$ gives a basic measure of the device's ability to influence rotor torque and thus rotational speed or power output. Specifically, the suction coefficient is defined as follows:

$$
C_{S}=C_{l} \sin \alpha-C_{d} \cos \alpha
$$

where $C_{\mathrm{l}}$ and $\mathrm{C}_{\mathrm{d}}$ are respectively the section lift and drag coefficients and $\alpha$ is the airfoil angle of attack.

During normal turbine operation, the suction coefficient is positive, thus promoting rotor rotation and the production of power. A slight change in the suction coefficient effects minor torque changes (i.e., power modulation) and a large reduction, to a negative value, produces braking torque. As is obvious, to produce large negative $\mathrm{C}_{\mathrm{S}}$ values one must significantly drop the airfoil lift and increase the drag.

The following sections present the aerodynamic results obtained during the wind tunnel testing. A plot of the suction, lift, and drag coefficient performance is provided for each configuration, as a function of device deflection angle and angle of attack.

Unless indicated otherwise, the device lip length and vent angle can be assumed to be, respectively, 1\% and 40 degrees. In addition, it is important to note that the drag and suction coefficient data presented in the following sections have not been corrected for tare and interference effects.

\section{Impact of Lip Length on $\mathbf{5 0 \%}$ Chord Device}

The lip length of the $50 \%$ device was varied in an attempt to further improve performance or to possibly reduce the device chord while retaining performance. Figures 11-15 $(a, b$, and $c)$ show the effects of lip length as a function of device deflection and angle of attack.

In general, for angles of attack below approximately 18 degrees the impact of lip length is minimal for device deflections less than about 7.5 degrees. However, as Figures $11 \mathrm{a}$ and $12 \mathrm{a}$ illustrate, the longer lip produces a more negative suction coefficient at higher angles of attack.

Figures $13 \mathrm{a}$ and $14 \mathrm{a}$ illustrate that device deflections of 30 and 60 degrees and a lip length of $5 \%$ produce a notable drop in the suction coefficient over the entire angle of attack range examined. Interestingly, however, the impact of lip length is minimal once the device deflection reaches 90 degrees, as is shown in Figure 15a. 


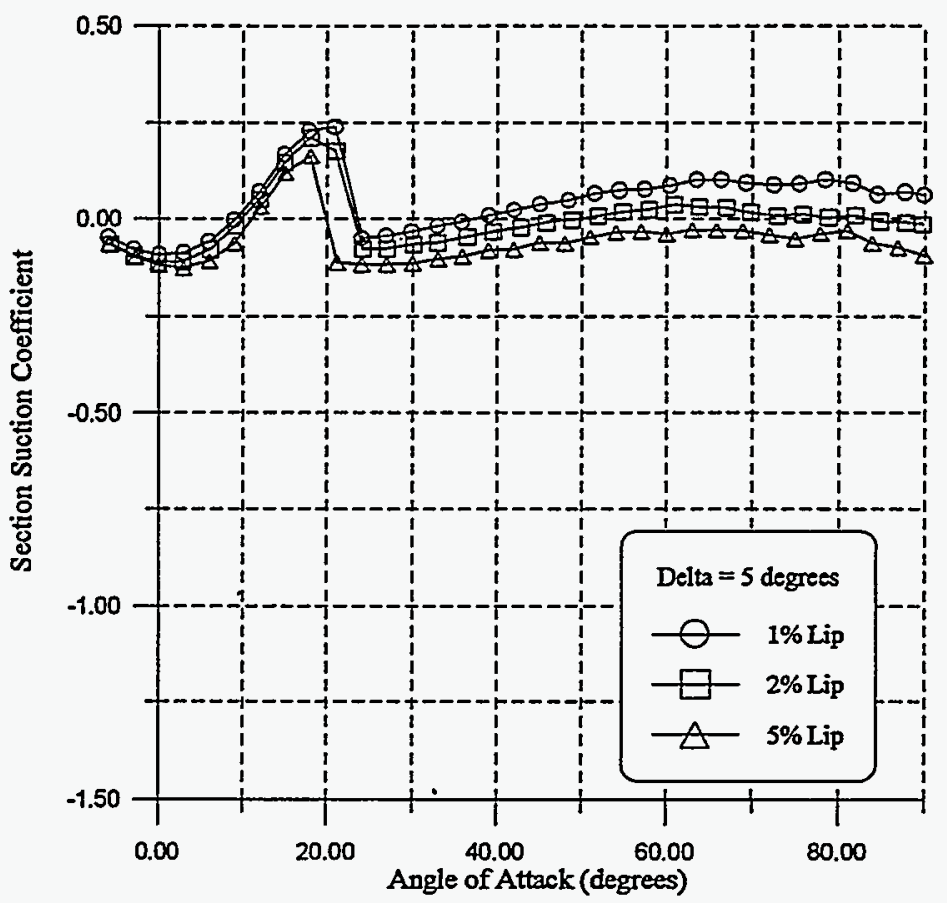

Figure 11a. Suction coefficient behavior

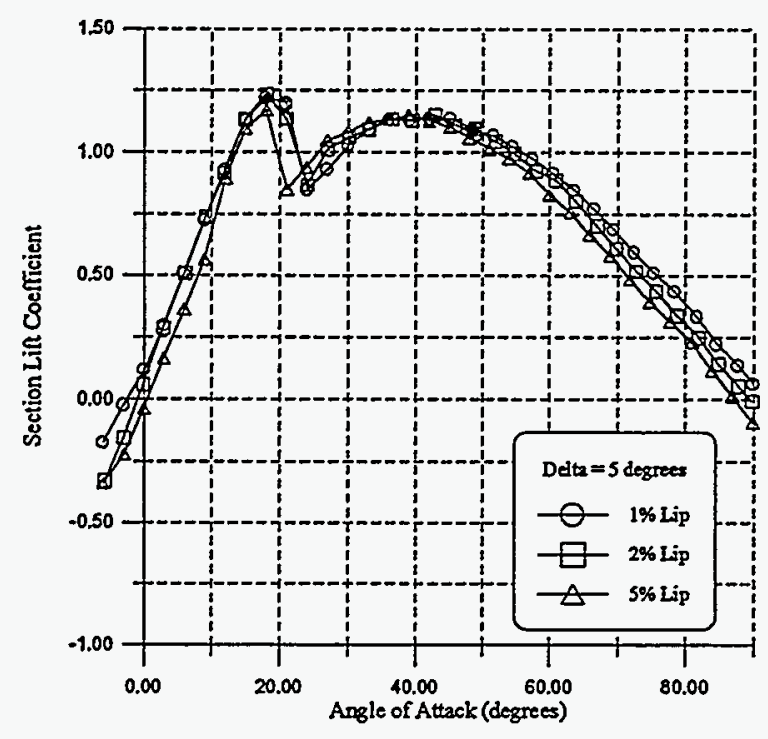

Figure 11b. Lift coefficient behavior

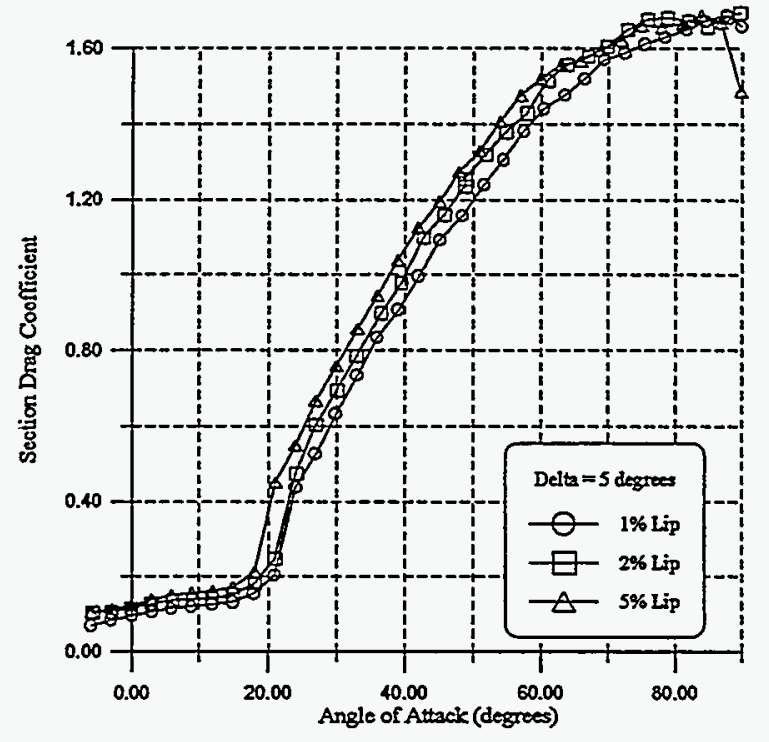

Figure 11c. Drag coefficient behavior

Figure 11. Impact of lip length on $\mathbf{5 0 \%}$ chord device at a delta of 5 degrees 


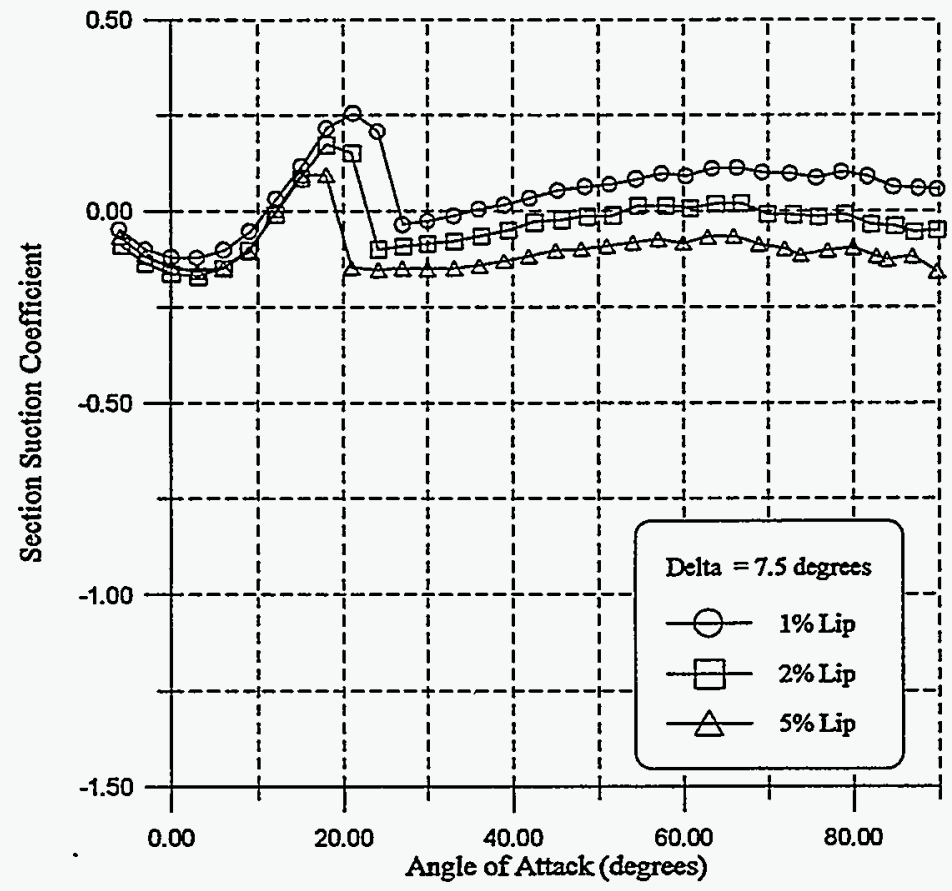

Figure 12a. Suction coefficient behavior

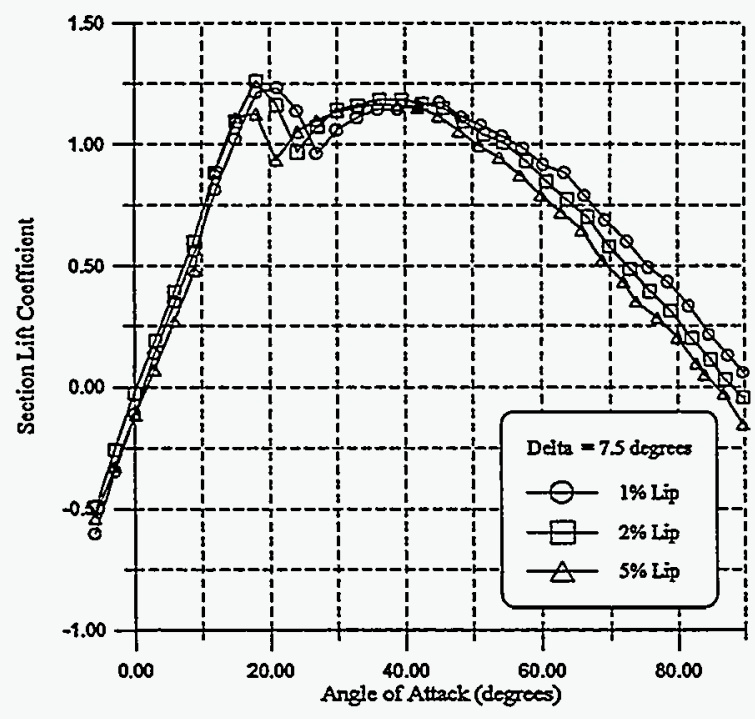

Figure 12b. Lift coefficient behavior

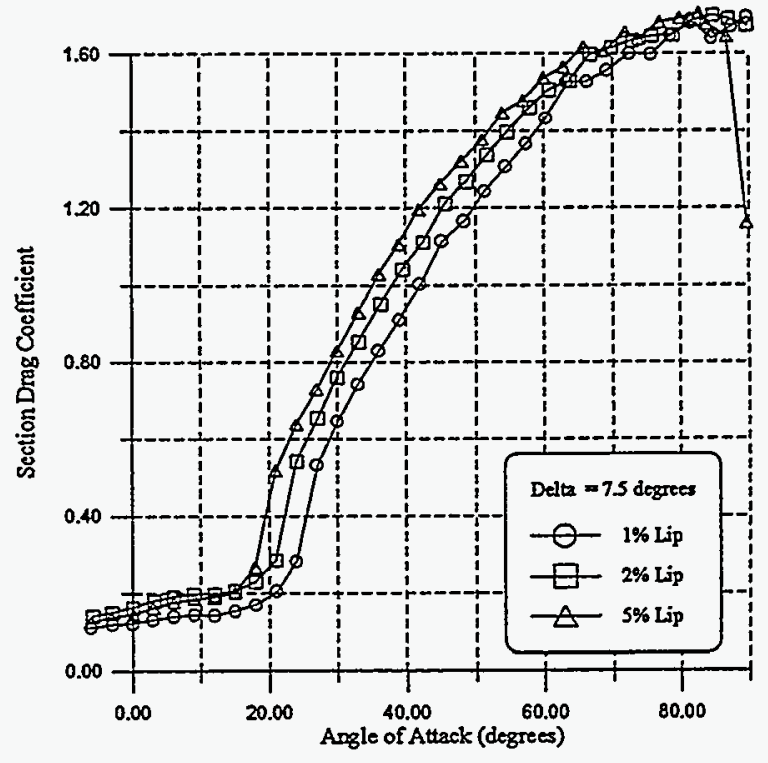

Figure 12c. Drag coefficient behavior

Figure 12. Impact of lip length on $\mathbf{5 0 \%}$ chord device at a delta of $\mathbf{7 . 5}$ degrees 


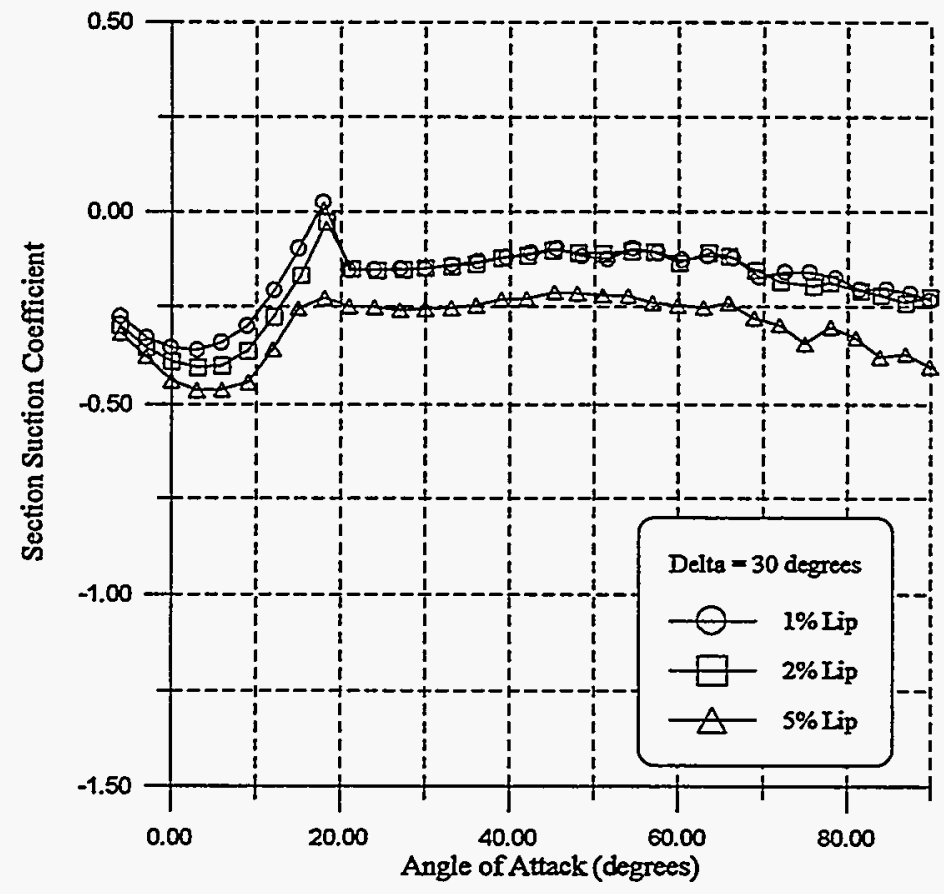

Figure 13a. Suction coefficient behavior

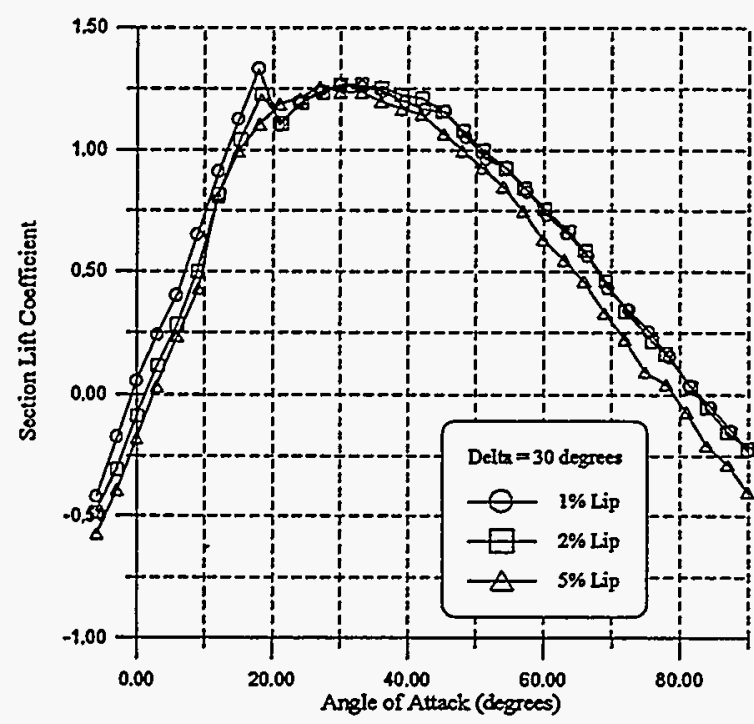

Figure 13b. Lift coefficient behavior

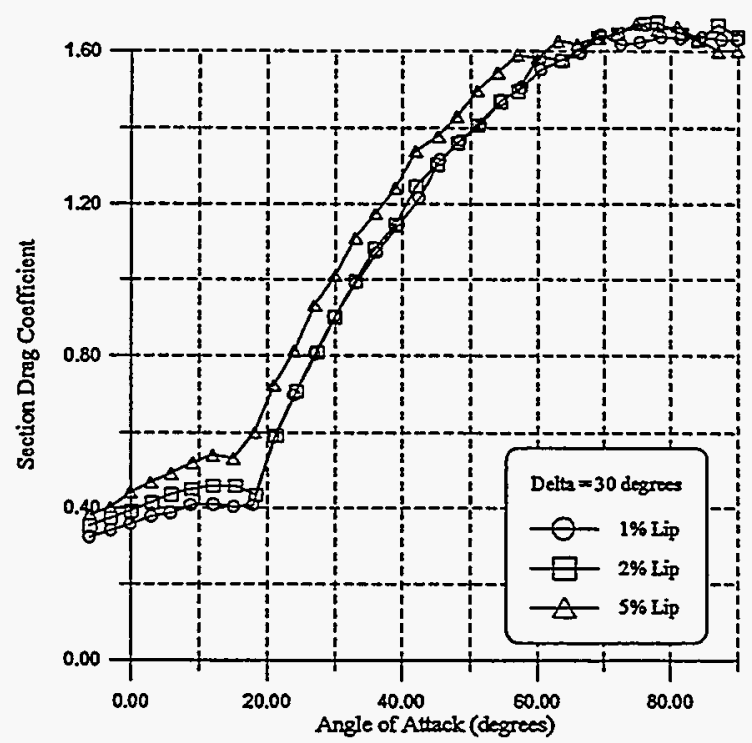

Figure 13c. Drag coefficient behavior

Figure 13. Impact of lip length on $\mathbf{5 0 \%}$ chord device at a delta of $\mathbf{3 0}$ degrees 


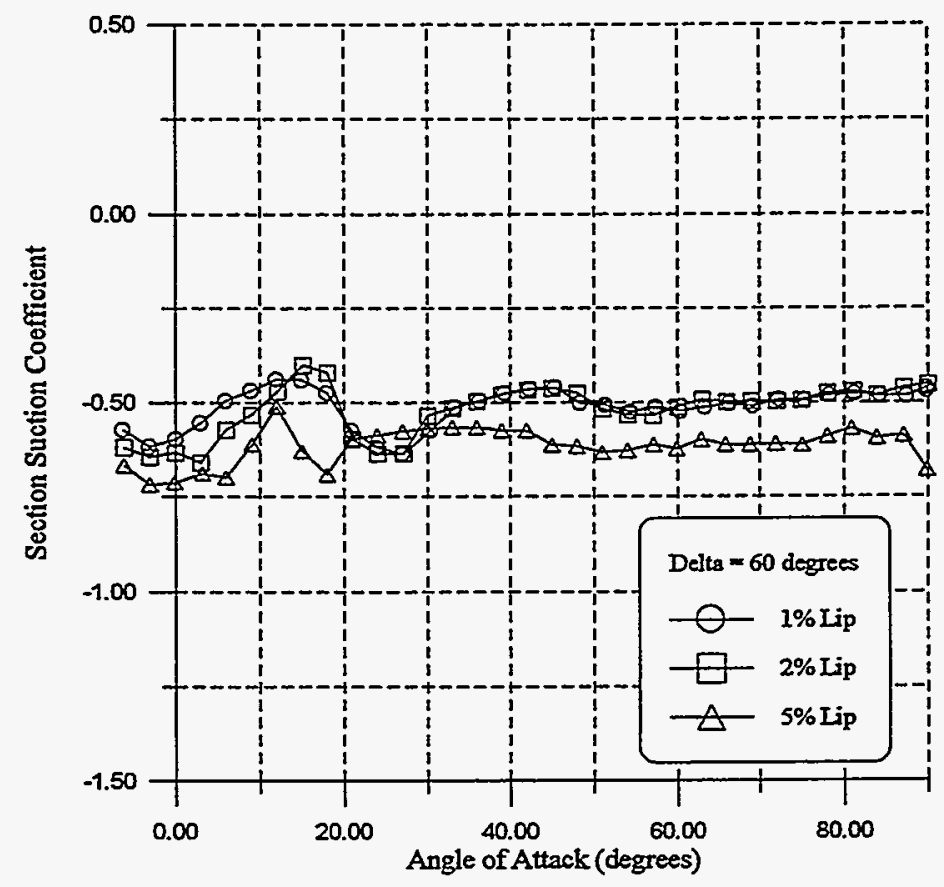

Figure 14a. Suction coefficient behavior

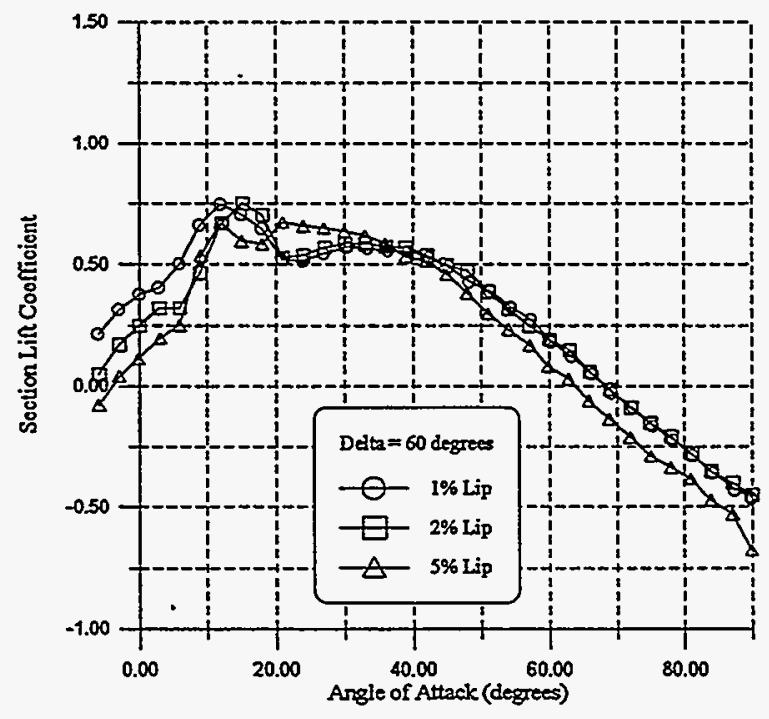

Figure 14b. Lift coefficient behavior

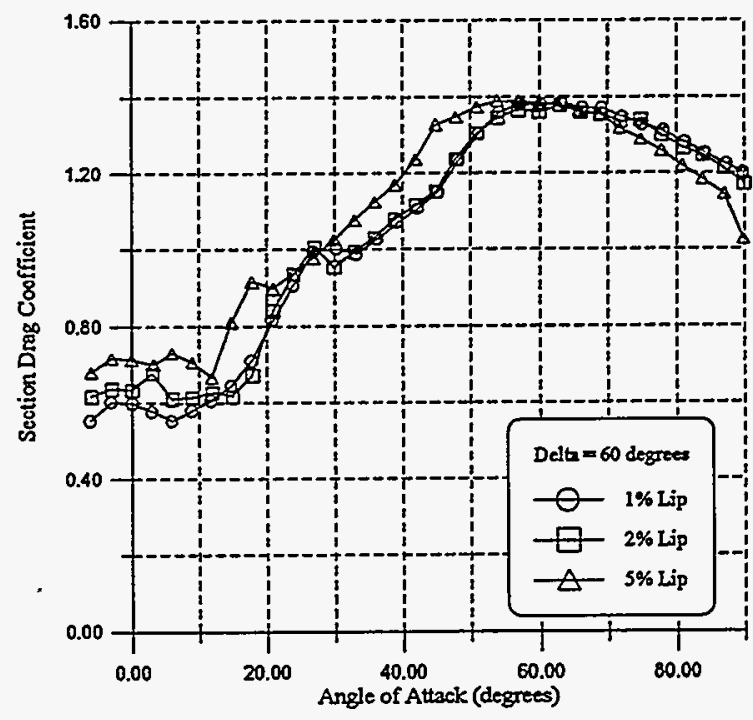

Figure 14c. Drag coefficient behavior

Figure 14. Impact of lip length on $50 \%$ chord device at a delta of 60 degrees 


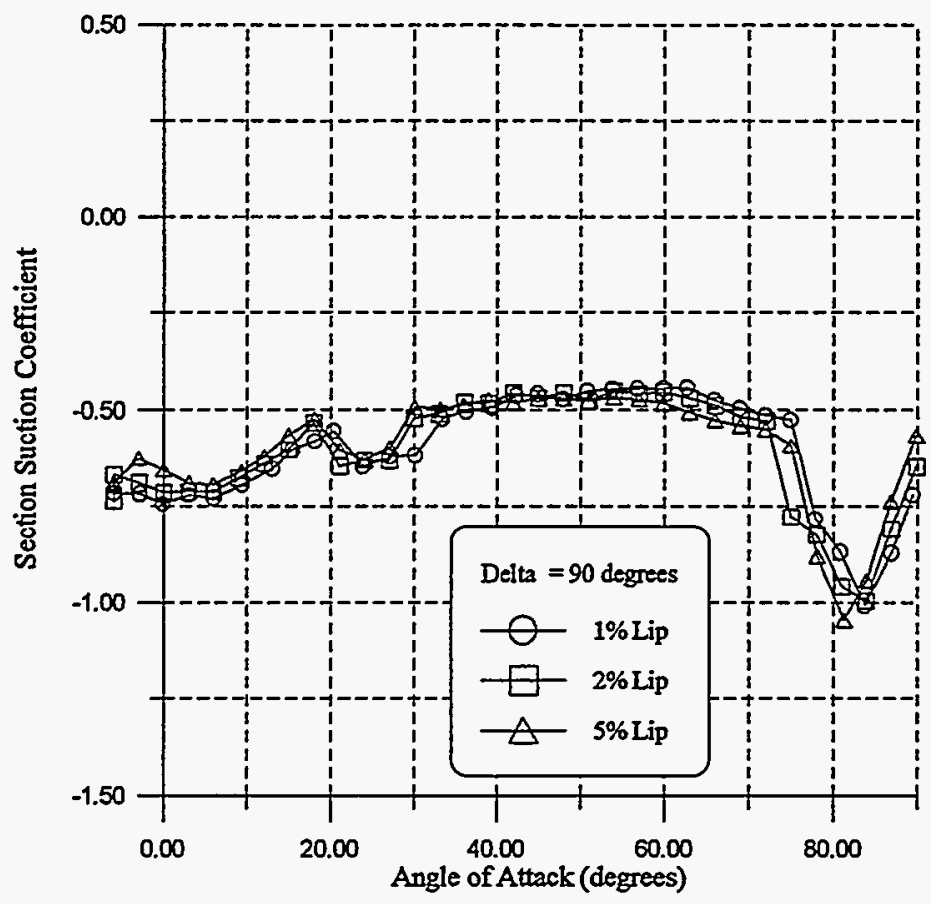

Figure 15a. Suction coefficient behavior

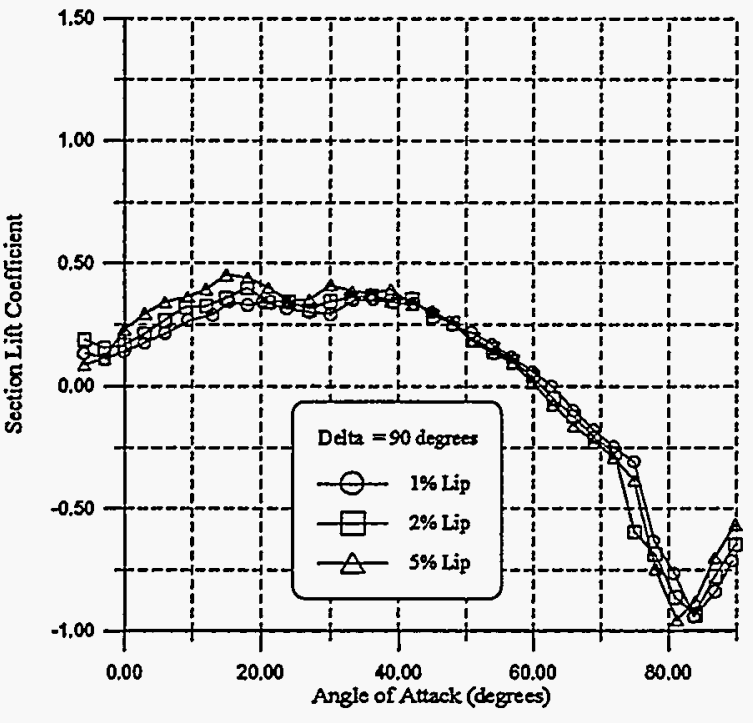

Figure 15b. Lift coefficient behavior

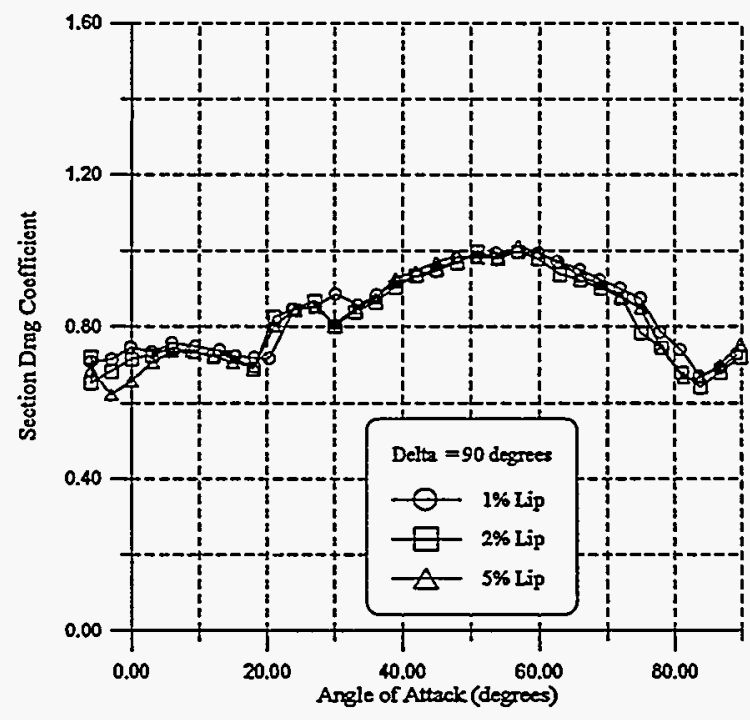

Figure 15c. Drag coefficient behavior

Figure 15. Impact of lip length on $50 \%$ chord device at a delta of 90 degrees 


\section{Vent Angle Effects on the $\mathbf{5 0 \%}$ Chord Device}

The effect of device actuation such that the flow between the lower and upper surfaces is allowed to freely mix was next examined. Figures 16-19 (, $\mathrm{b}$ and $\mathrm{c}$ ) show the effects of vent angle, as a function of device deflection and angle of attack, for the $50 \%$ chord device. The airfoil cove and device leading-edge geometry, as shown in Figures 2-5, allowed vent angles of $0,15,30$ and 40 degrees to be evaluated. Recall that the Phase 1 configuration, as shown in Figures 1 and 2, vents at a device deflection of 40 degrees.

Figures $16 \mathrm{a}$ and $17 \mathrm{a}$ illustrate that the benefit of venting before the device has deflected 40 degrees is minimal for angles of attack less than about 25 degrees. A small, favorable effect on the suction coefficient is observed at angles of attack greater than 25 degrees.

Figures $18 \mathrm{a}$ and $19 \mathrm{a}$ show that the original configuration (i.e., 40 degree vent) device performs better, on the average, than the new $(0,15$ and 30 degree) venting configurations. The performance difference is most noticeable for a device deflection of 60 degrees, between 10 and 35 degrees angle of attack. As Figure 19a shows, a 40 degree vent configuration offers substantially improved suction coefficient performance over the entire angle of attack range once the device is deflected 90 degrees. 


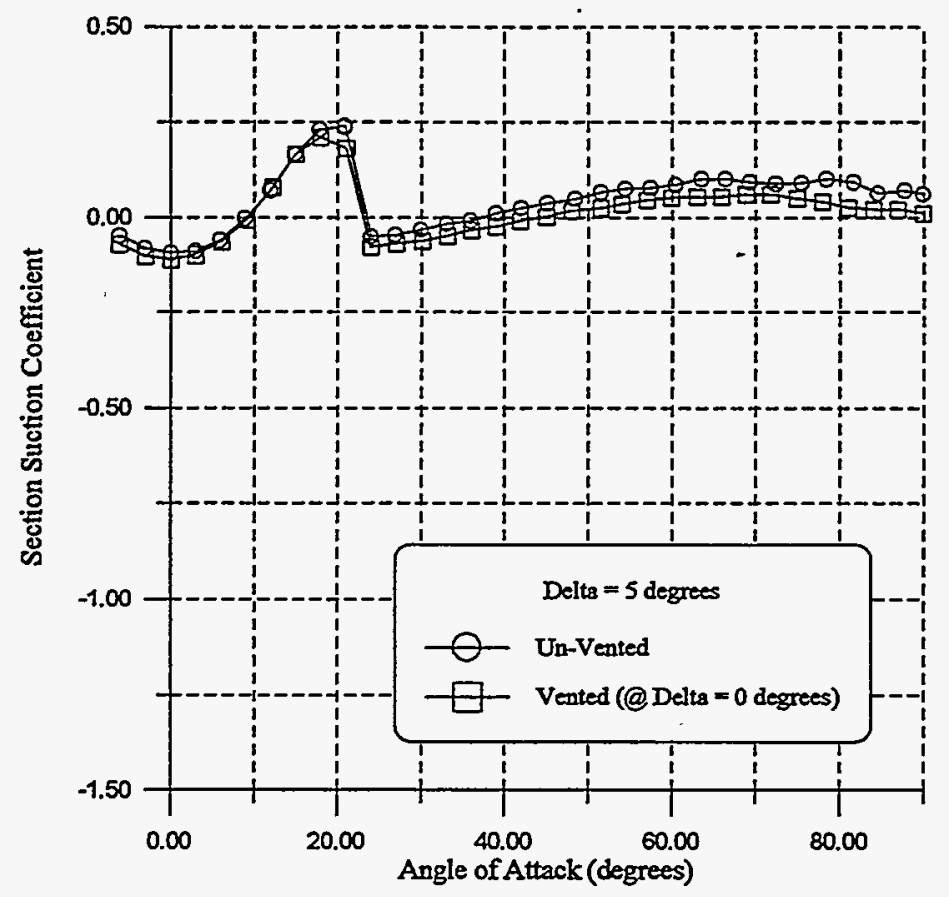

Figure 16a. Suction coefficient behavior

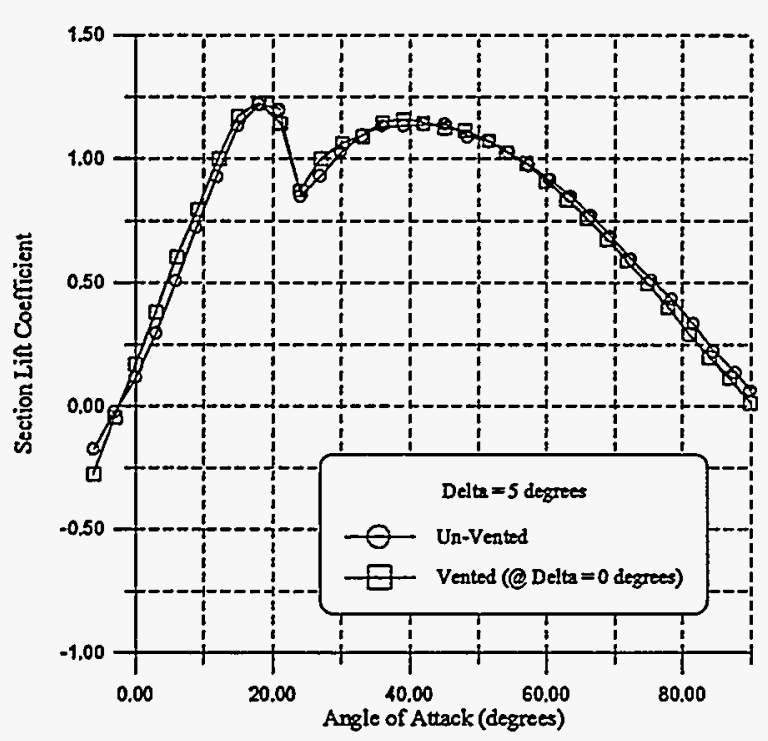

Figure 16b. Lift coefficient behavior

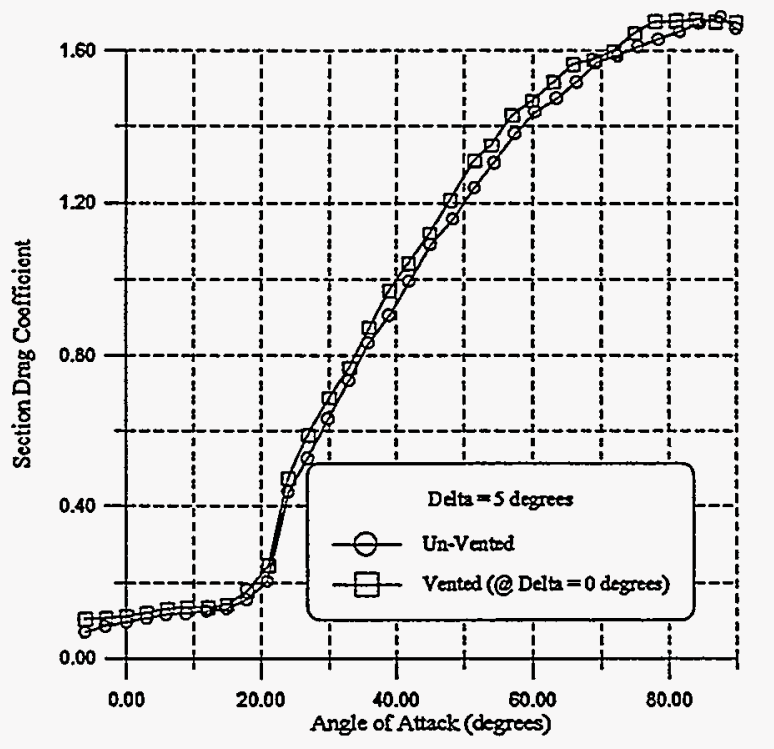

Figure 16c. Drag coefficient behavior

Figure 16. Impact of vent angle on $\mathbf{5 0 \%}$ chord device at a delta of $\mathbf{5}$ degrees 


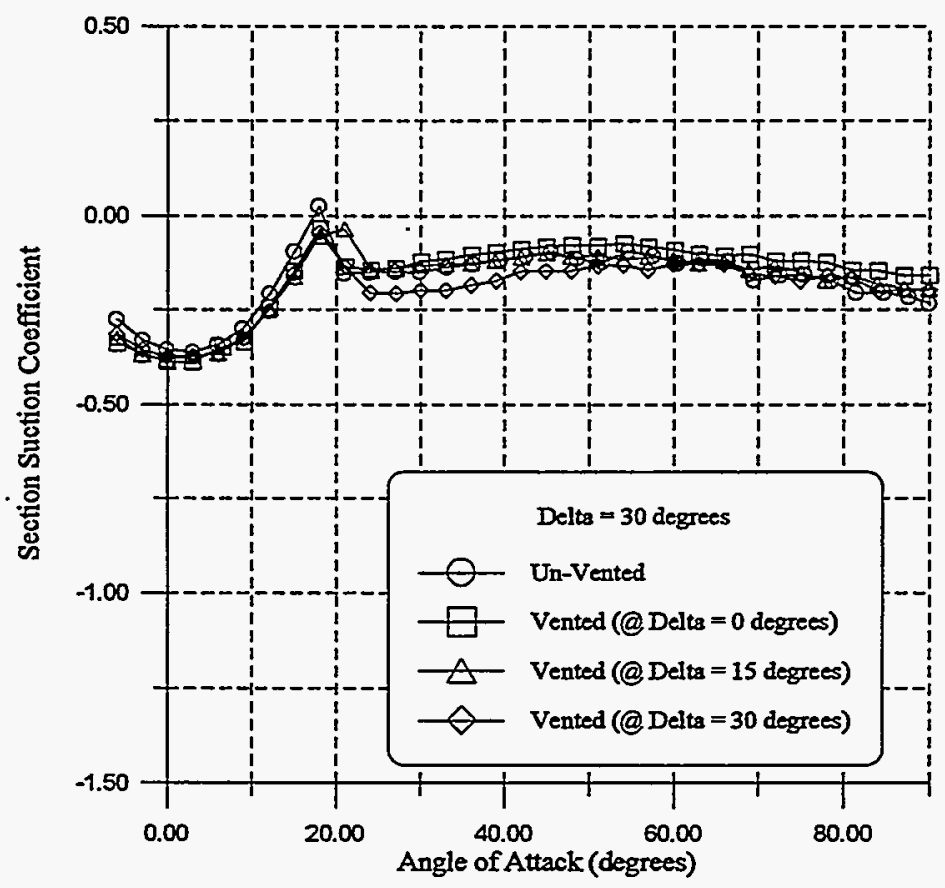

Figure 17a. Suction coefficient behavior

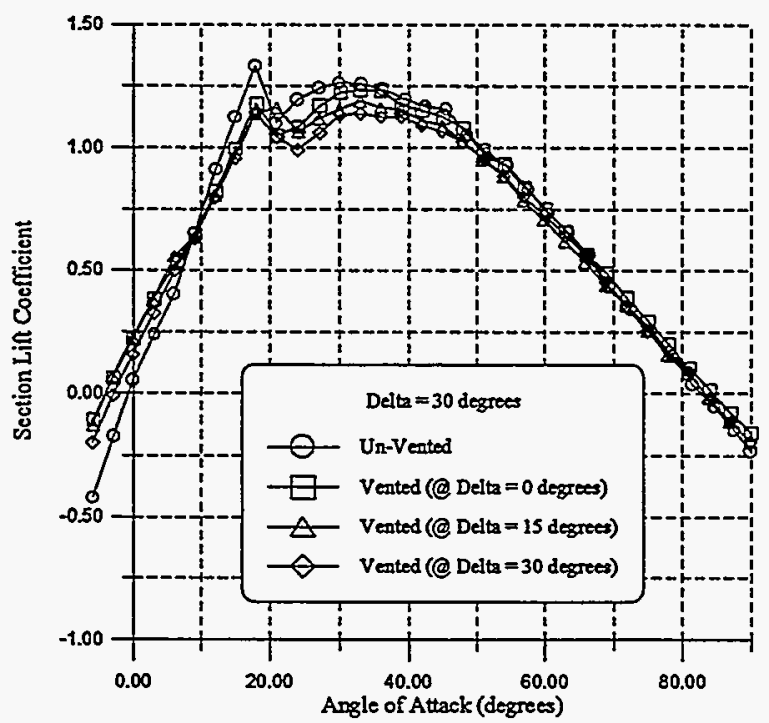

Figure 17b. Lift coefficient behavior

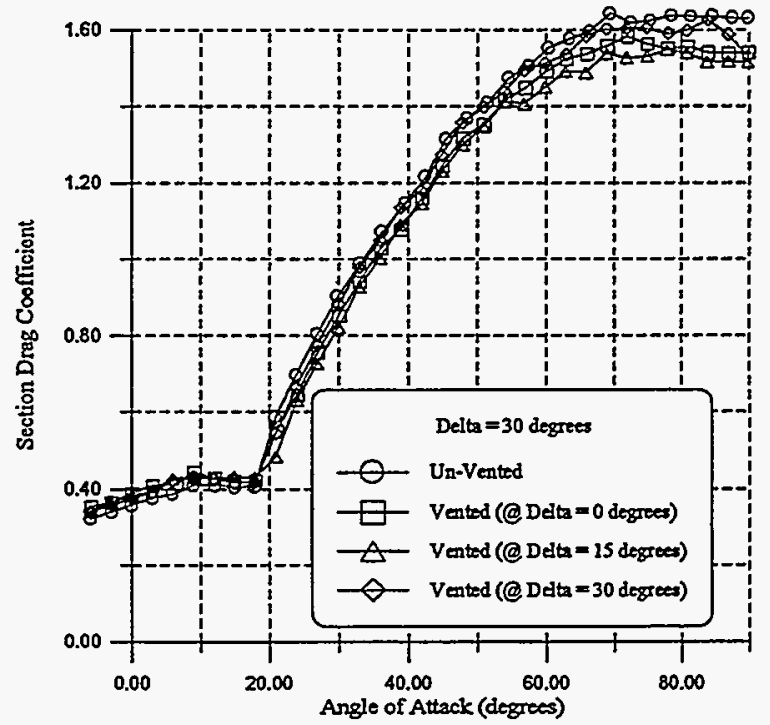

Figure 17c. Drag coefficient behavior

Figure 17. Impact of vent angle on $\mathbf{5 0 \%}$ chord device at a delta of $\mathbf{3 0}$ degrees 


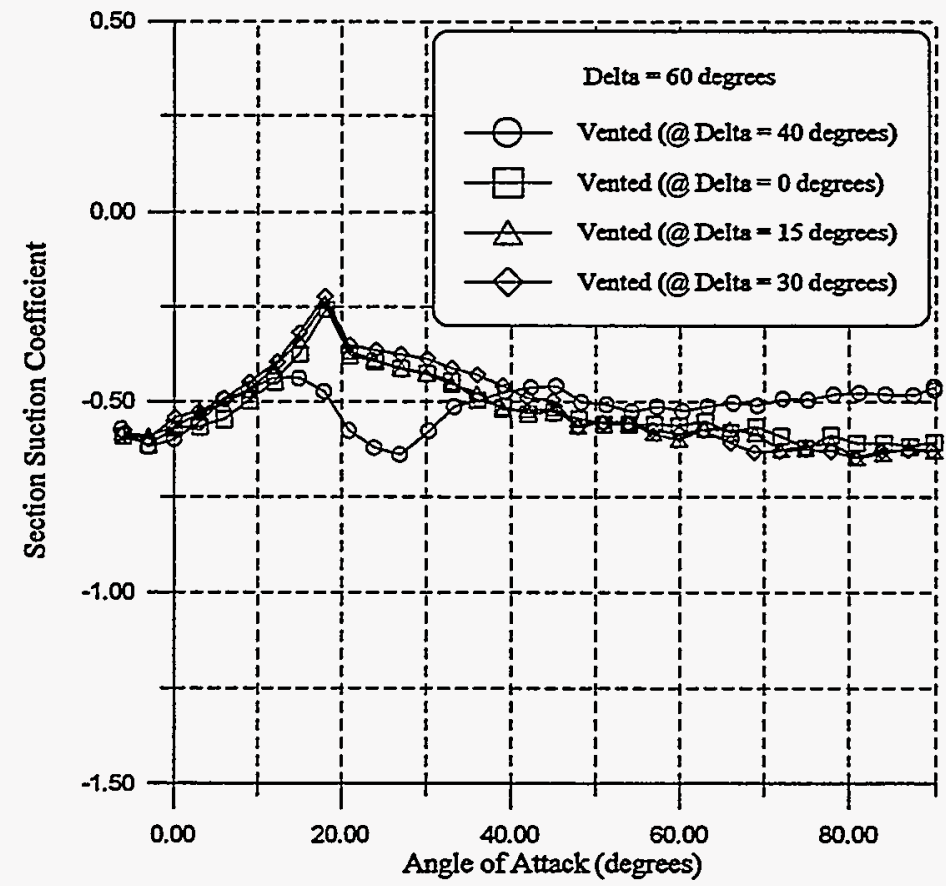

Figure 18a. Suction coefficient behavior

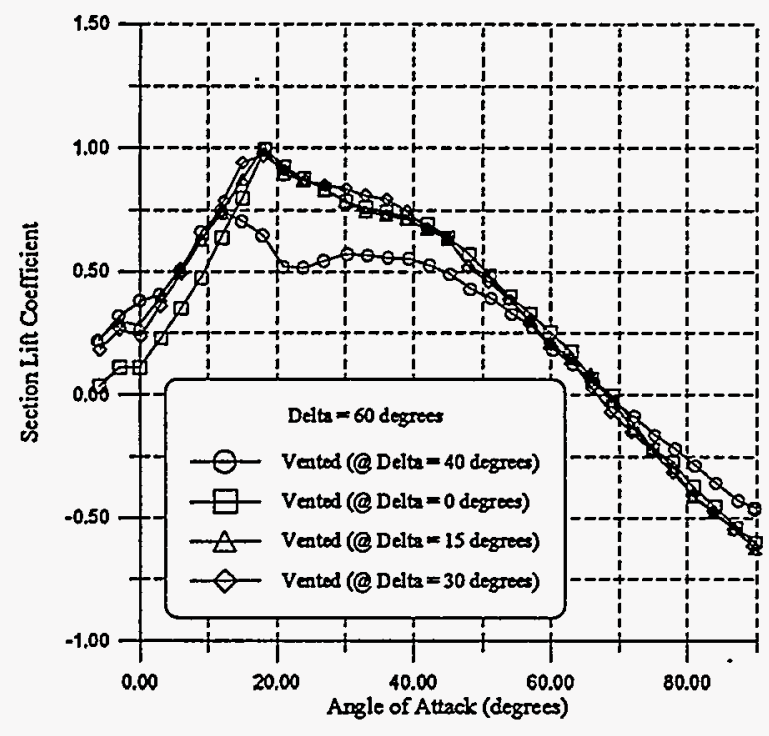

Figure 18b. Lift coefficient behavior

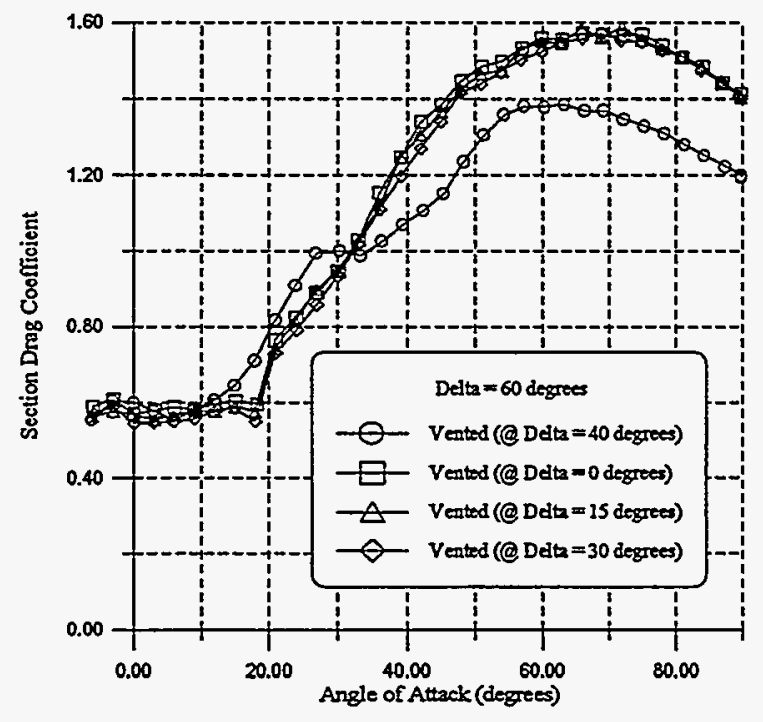

Figure 18c. Drag coefficient behavior

Figure 18. Impact of vent angle on $50 \%$ chord device at a delta of 60 degrees 


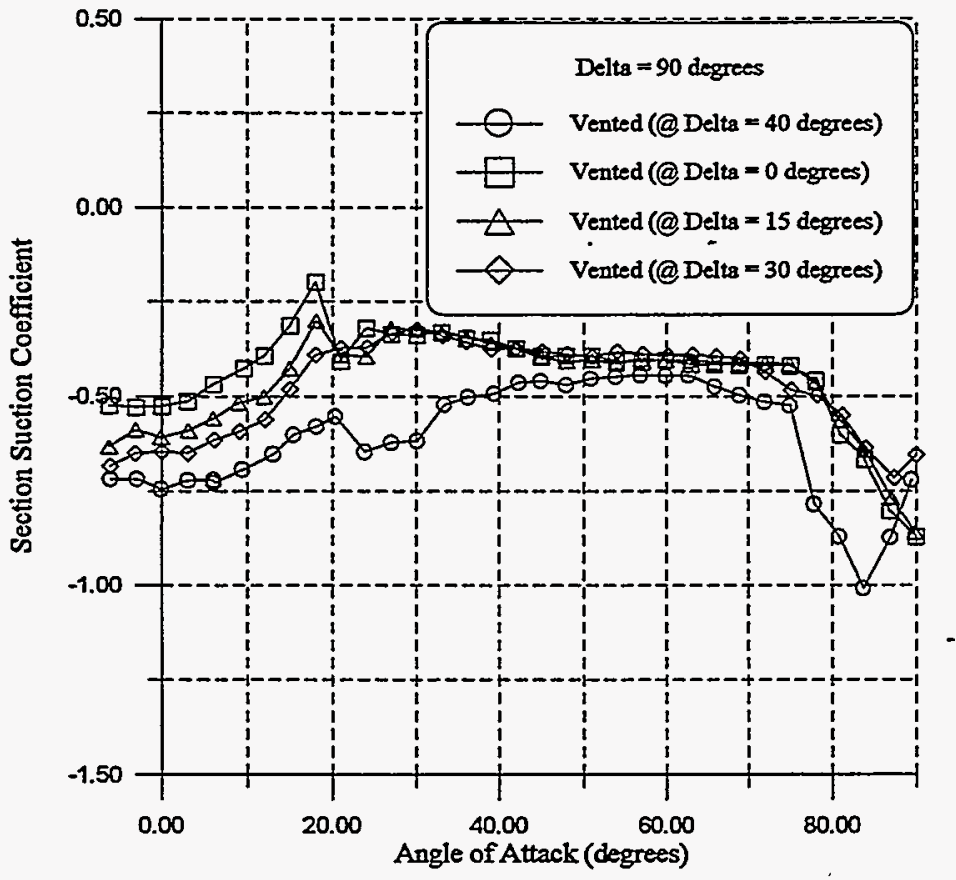

Figure 19a. Suction coefficient behavior

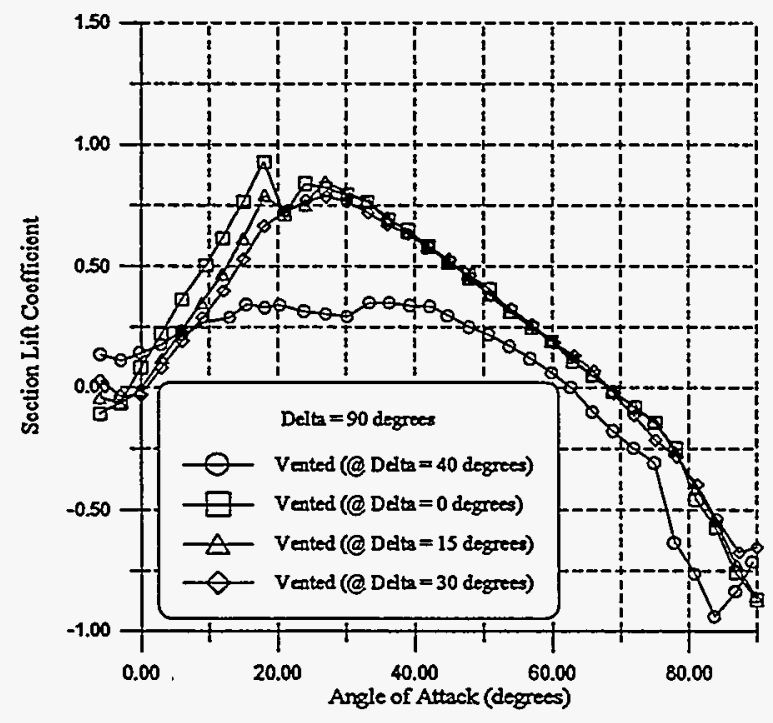

Figure 19b. Lift coefficient behavior

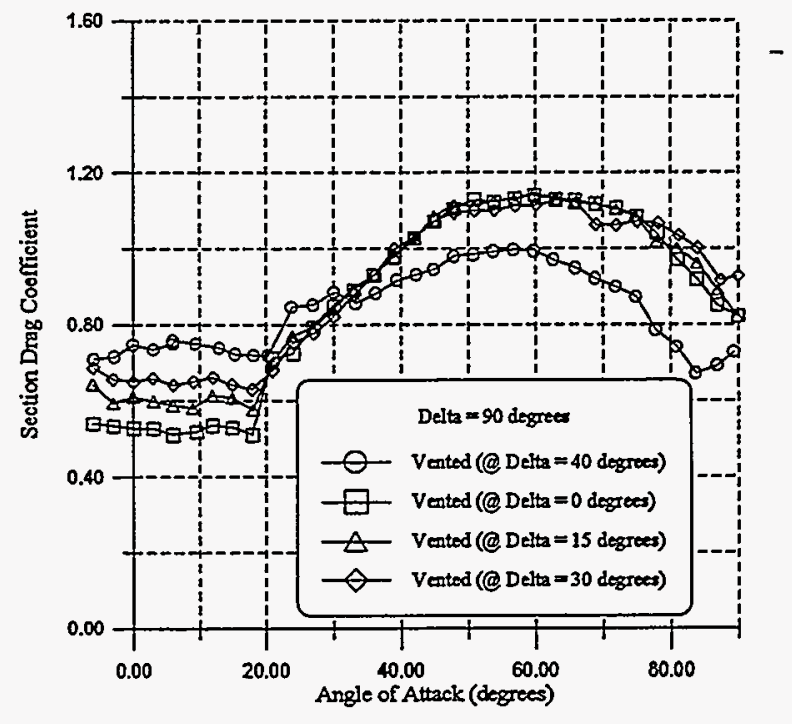

Figure 19c. Drag coefficient behavior

Figure 19. Impact of vent angle on $\mathbf{5 0 \%}$ chord device at a delta of $\mathbf{9 0}$ degrees 


\section{Vent Angle Effects on the $\mathbf{4 0 \%}$ Chord Device}

The impact of vent angle was next evaluated on the $40 \%$ chord device. Figures 20-22 (a, b, and c) show the effect of vent angle, as a function of device deflection and angle of attack. Two vent angles, 40 and 0 degrees, were evaluated. Schematic diagrams (Figures 6 and 7) show the corresponding configurations.

For a device deflection of 30 degrees, immediate venting slightly increases the suction coefficient (i.e., it becomes more positive) for angles of attack greater than approximately 25 degrees. However, once the device deflection reaches 60 degrees, this trend reverses and the early ( 0 degree) venting device performs better over angles of attack between 50 and 90 degrees. As Figure 22a illustrates, the impact of vent angle is minimal for a device deflection of 90 degrees.

Overall, the impact of vent angle for the $40 \%$ chord device was less pronounced than that observed for the $50 \%$ chord device. 


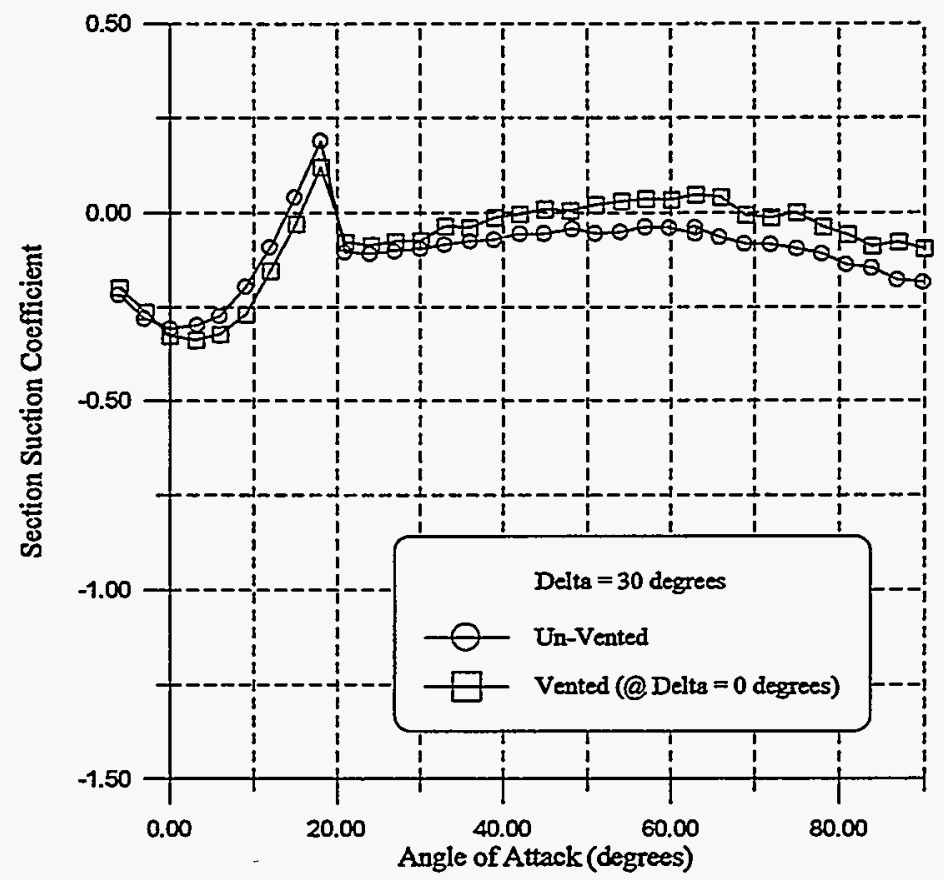

Figure 20a. Suction coefficient behavior

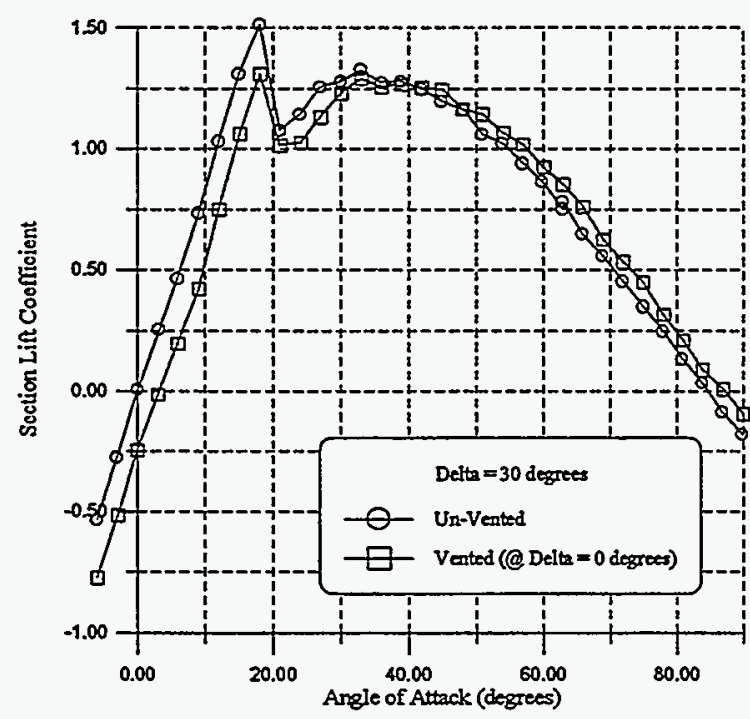

Figure 20b. Lift coefficient behavior

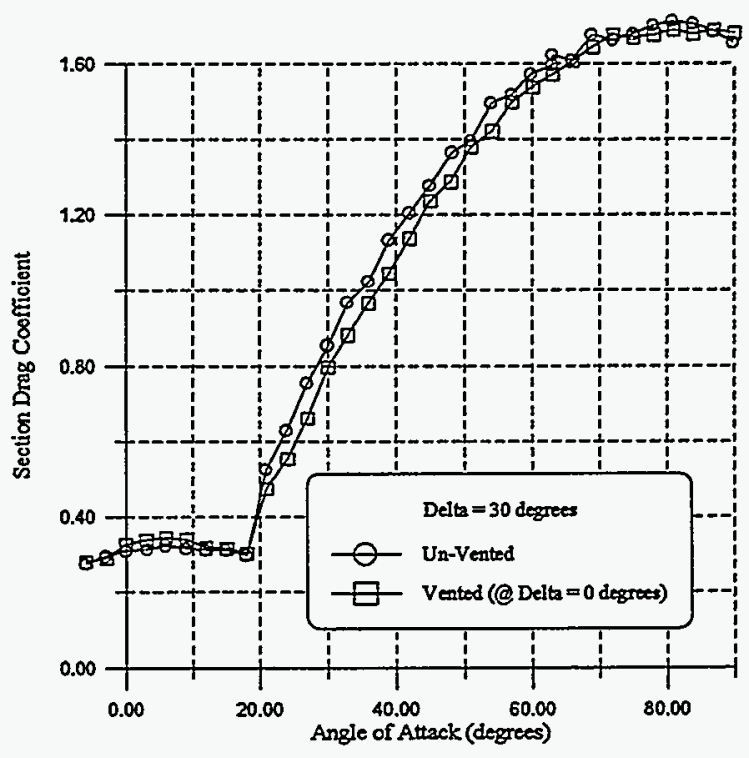

Figure 20c. Drag coefficient behavior

Figure 20. Impact of vent angle on $\mathbf{4 0 \%}$ chord device at a delta of $\mathbf{3 0}$ degrees 


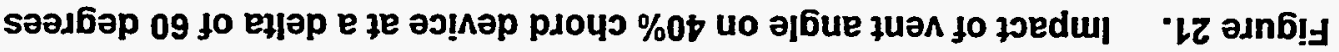

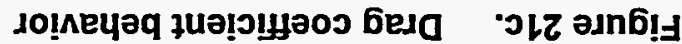

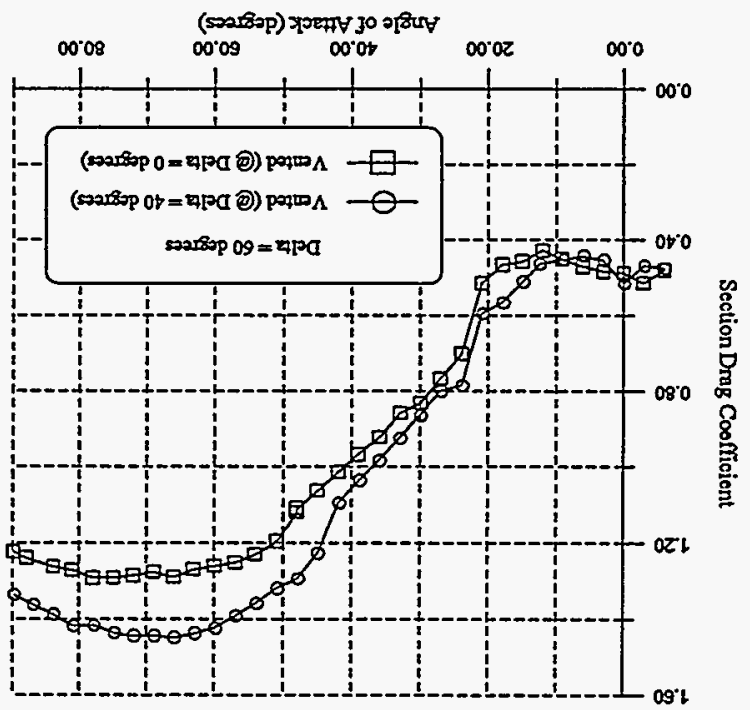

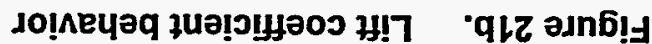

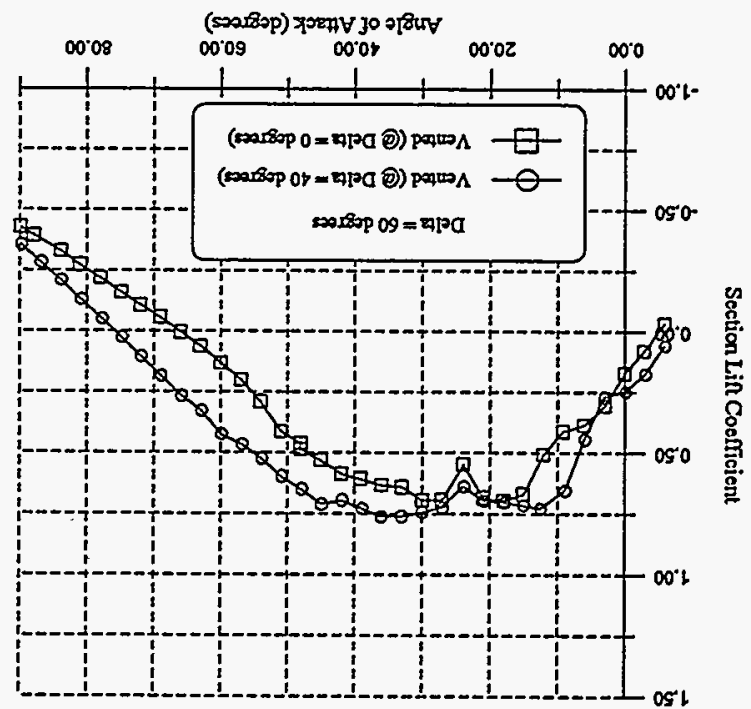

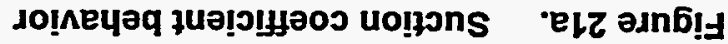

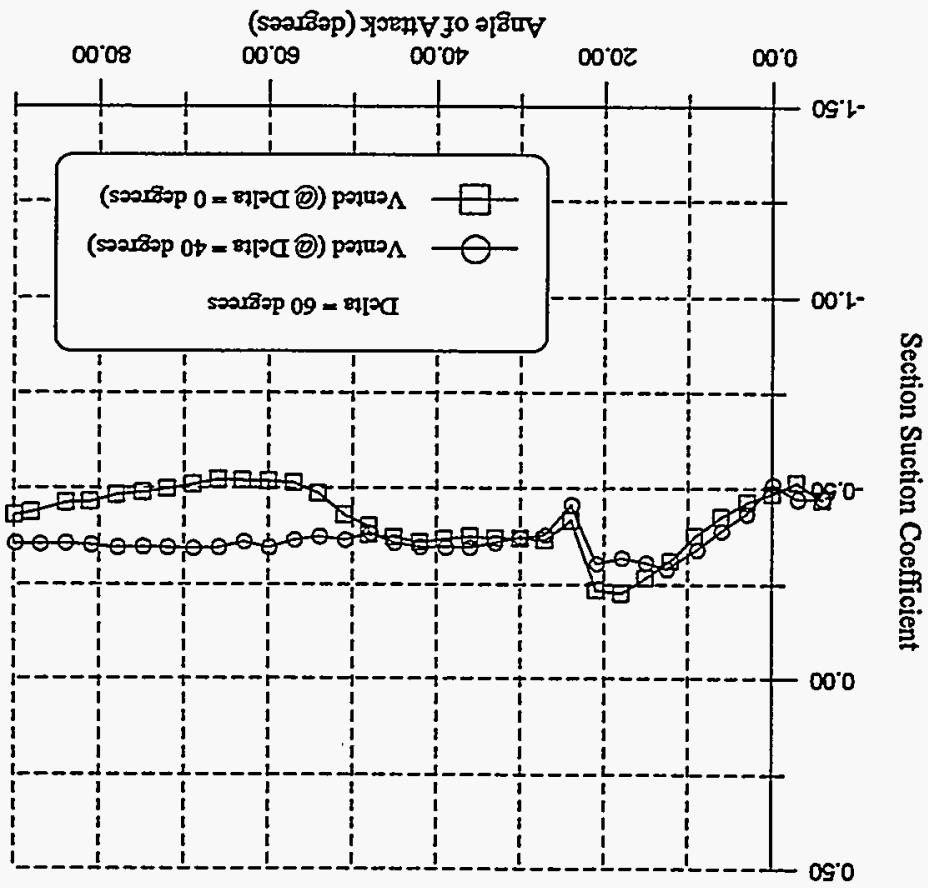




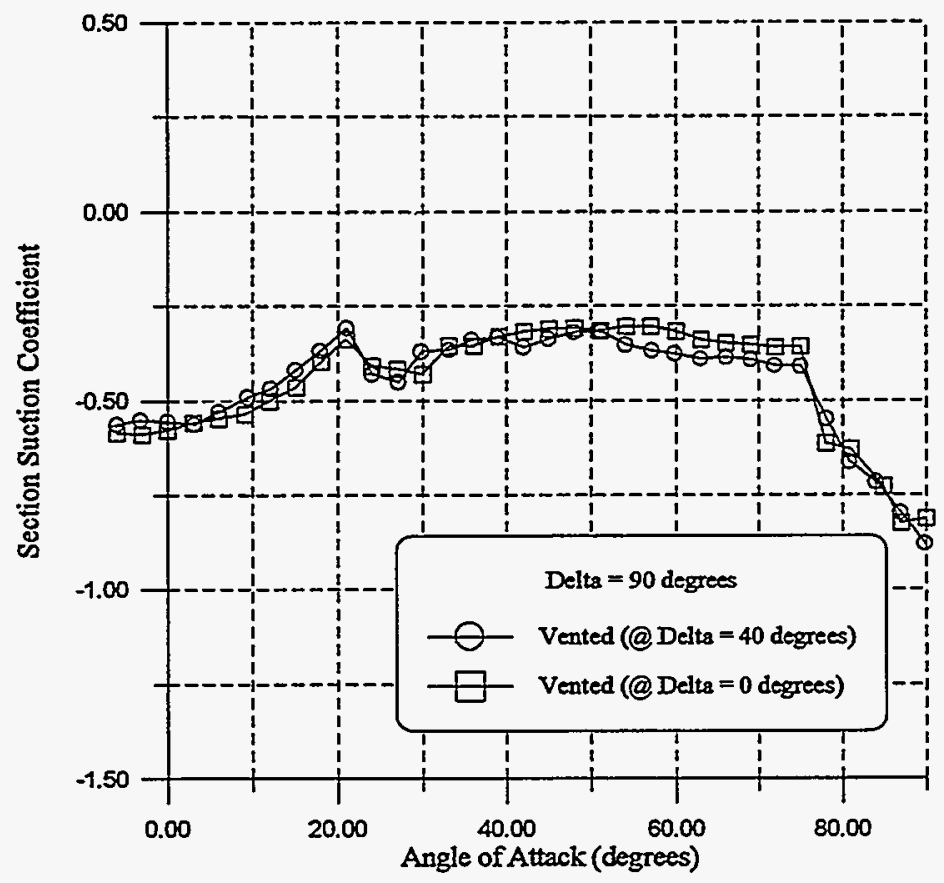

Figure 22a. Suction coefficient behavior

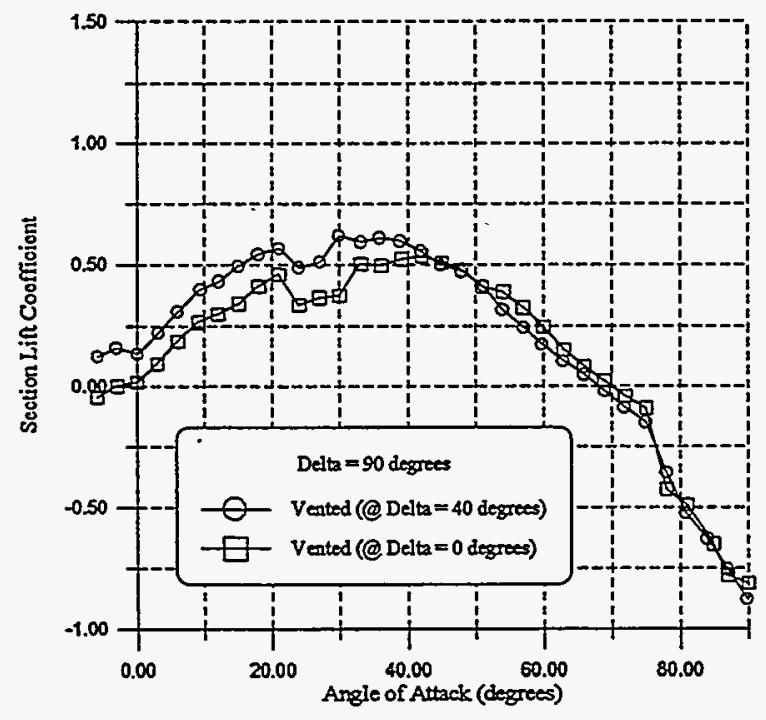

Figure 22b. Lift coefficient behavior

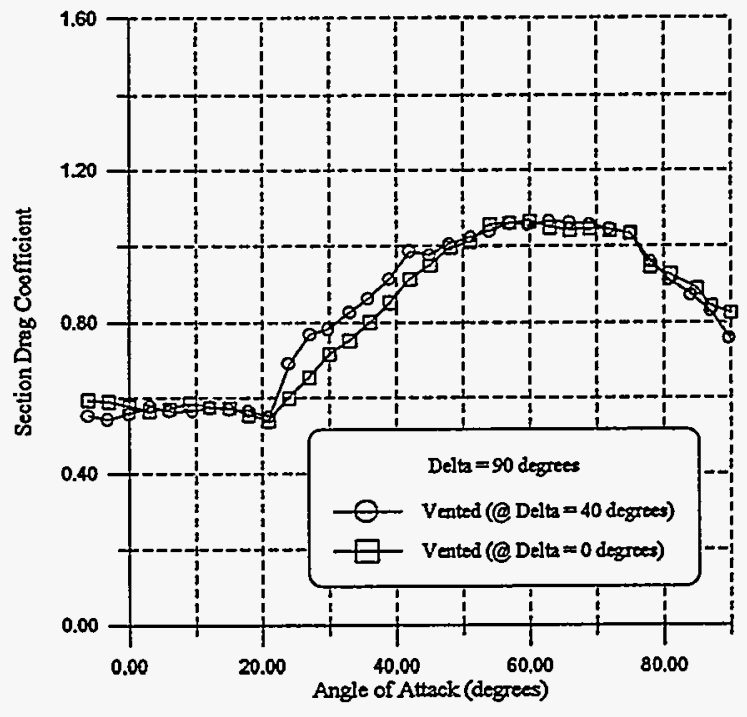

Figure 22c. Drag coefficient behavior

Figure 22. Impact of vent angle on $\mathbf{4 0 \%}$ chord device at a delta of $\mathbf{9 0}$ degrees 


\section{Effect of Device Chord}

Figures 23-25 ( $a, b$, and c) show the effect of device chord, as a function of device deflection and angle of attack. Fifty percent, $40 \%$, and $30 \%$ chord devices, as shown respectively in Figures 2,6 and 8 , were evaluated.

For all device deflections examined, the effect of chord length is most significant at angles of attack below 20 degrees. Differences between the various chord devices become even more pronounced, over the entire angle of attack range, as the device deflection continues. As might be expected, the larger chord device produces the more negative suction coefficient values and, interestingly, the $30 \%$ and $40 \%$ chord devices behave similarly at angles of attack greater than about 30 degrees. 


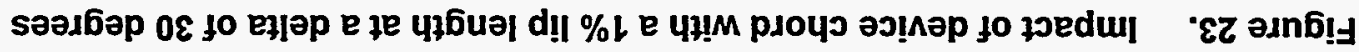

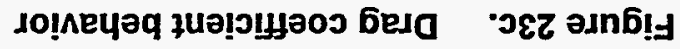

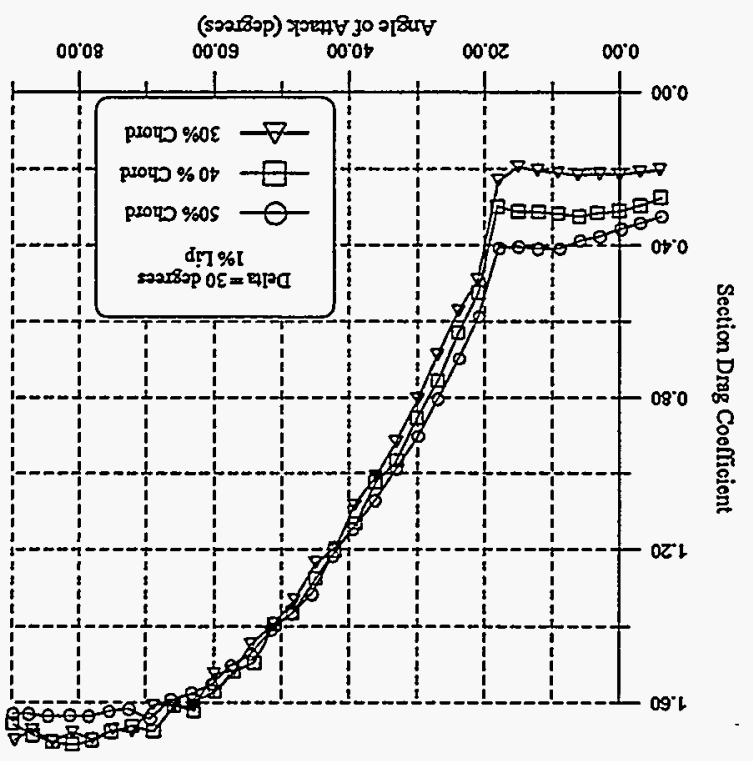

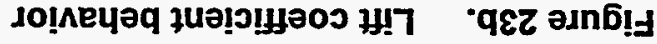

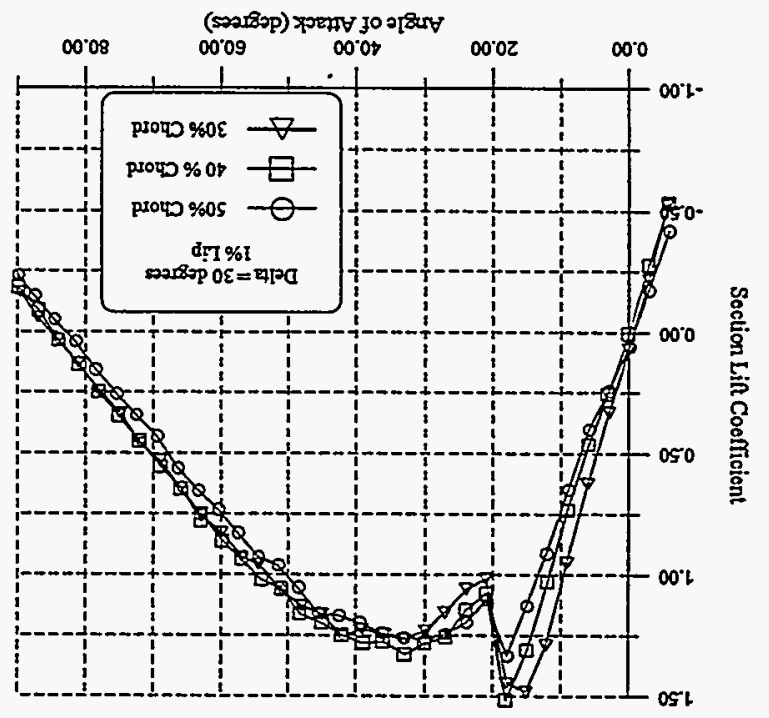

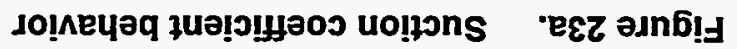

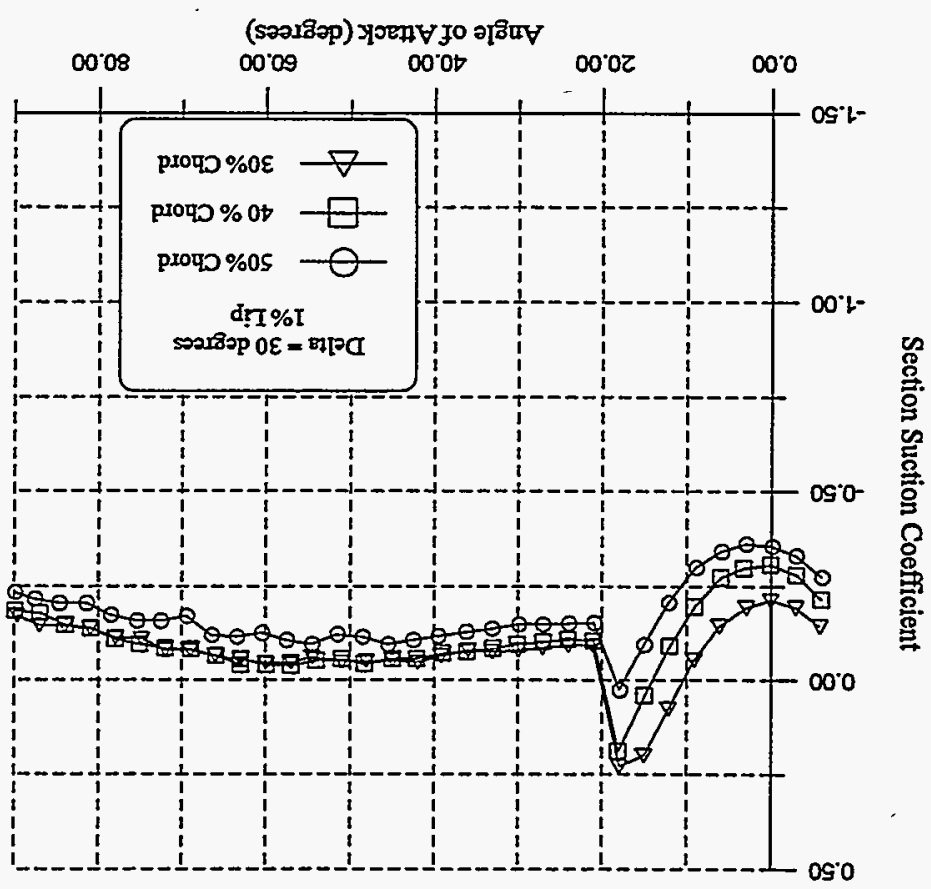




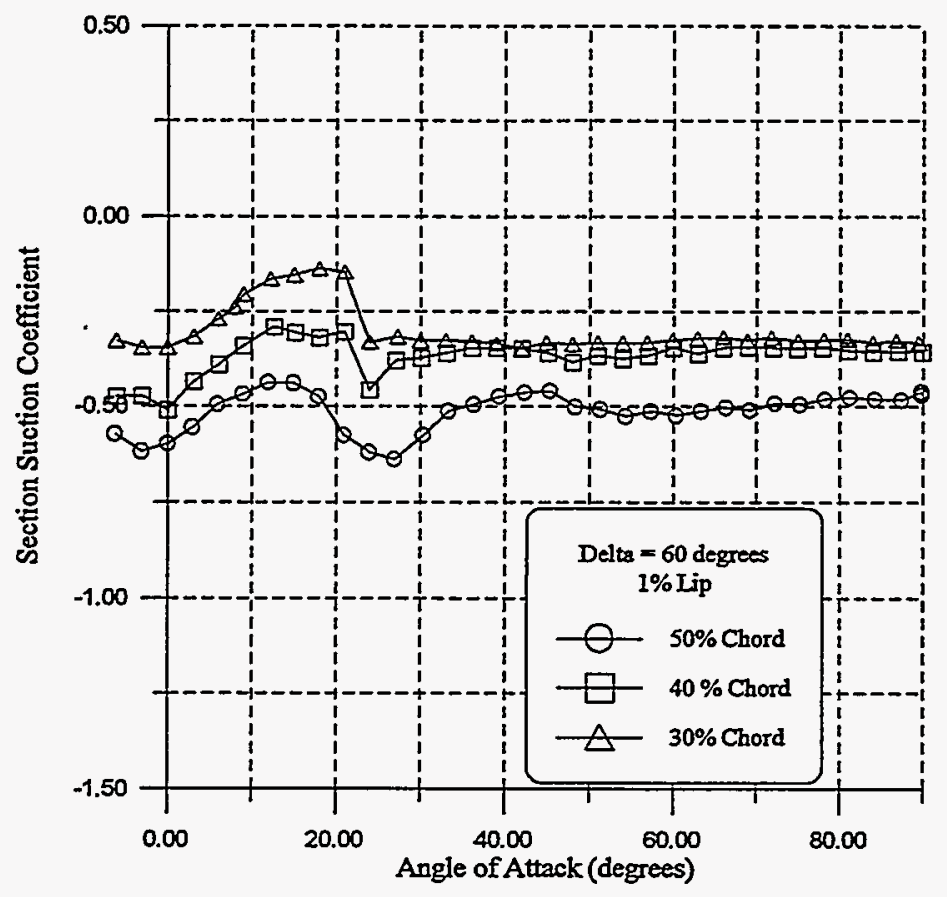

Figure 24a. Suction coefficient behavior

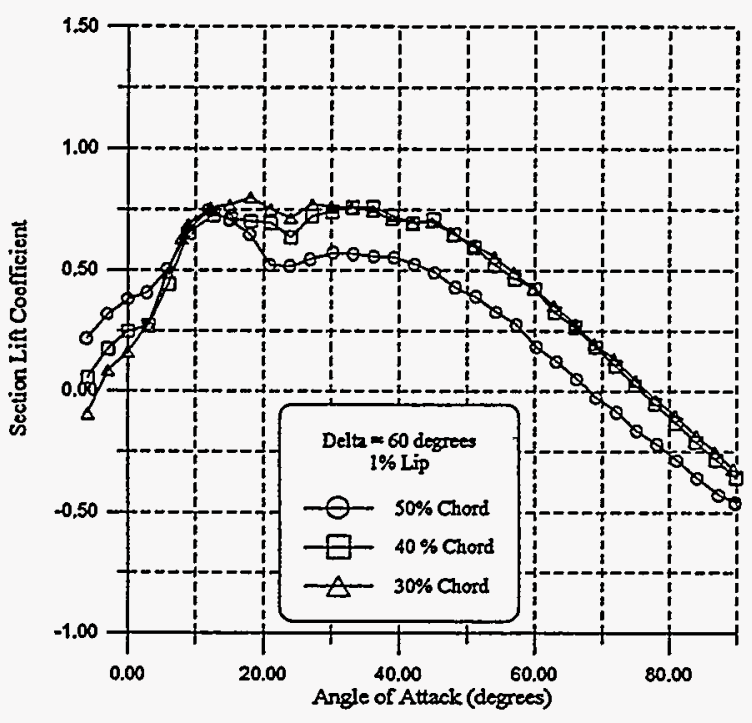

Figure 24b. Lift coefficient behavior

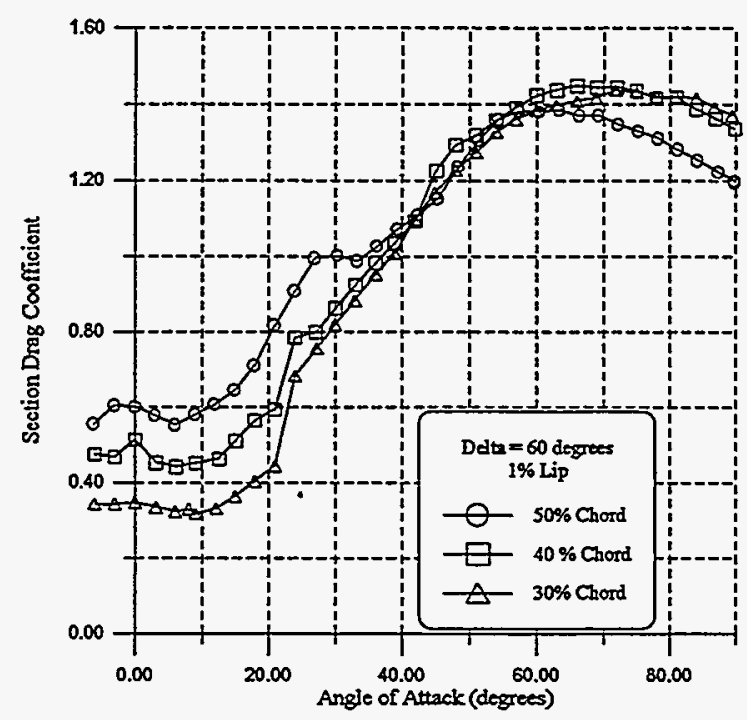

Figure 24c. Drag coefficient behavior

Figure 24. Impact of device chord with a 1\% lip length at a delta of 60 degrees 


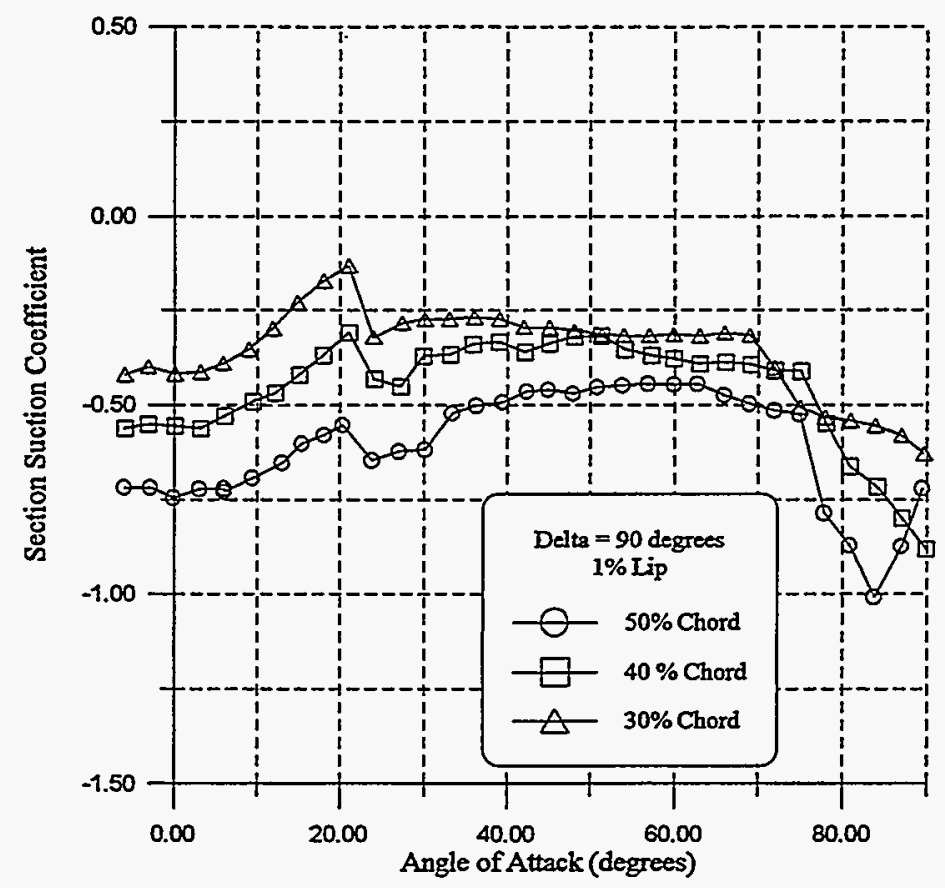

Figure 25a. Suction coefficient behavior

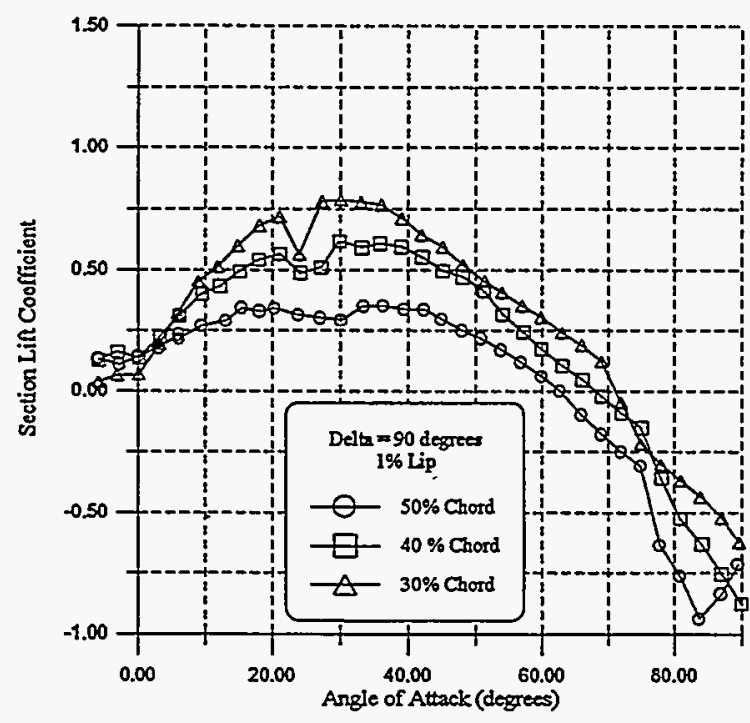

Figure 25b. Lift coefficient behavior

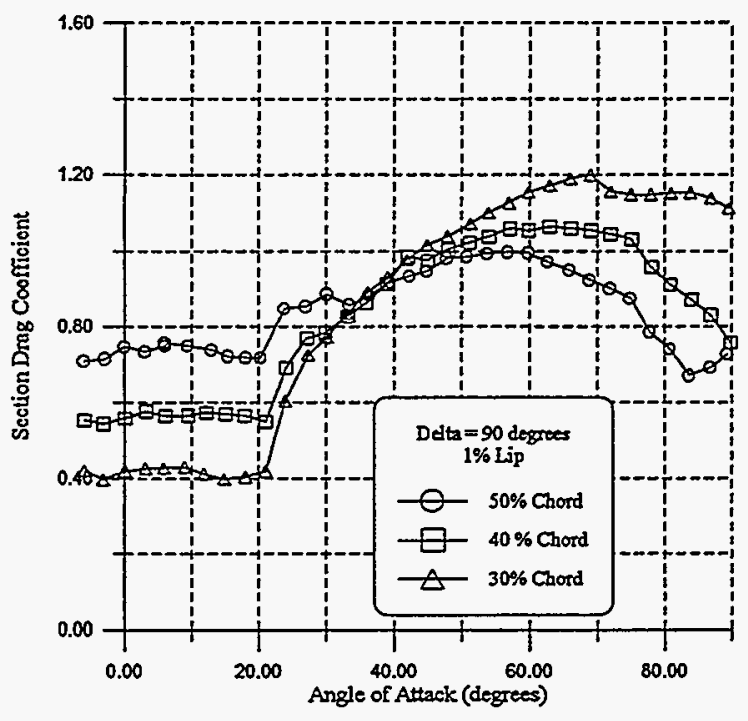

Figure 25c. Drag coefficient behavior

Figure 25. Impact of device chord with a 1\% lip length at a delta of 90 degrees 


\section{Normalized Device Effectiveness}

Figures 23-25 ( $a, b$, and $c)$, identified the direct effect of device chord on aerodynamic performance. This data shows that the $50 \%$ chord device produces the most negative suction coefficient behavior over the widest range of operating conditions. However, the efficiency of this device is not defined. It is conceivable that a shorter chord device might yield a greater relative change in the suction coefficient for its size, and hence be more effective. As a result, a measure of the relative efficiency or effectiveness of a given chord device is needed.

A new term has been defined to give an indication of the device efficiency. Specifically, the normalized device effectiveness $(\eta)$ is defined as follows,

$$
\eta=\Delta \mathrm{C}_{\mathrm{s}} / \mathrm{c}_{\mathrm{c}}
$$

where

$$
\Delta \mathrm{C}_{\mathrm{s}}=\mathrm{C}_{\mathrm{s} \text { (with-device) }}-\mathrm{C}_{\mathrm{s} \text { (no-device) }}
$$

This coefficient (i.e., $\eta$ ), with respect to the device chord, gives a normalized measure of the suction coefficient change from the basic no-device (i.e., $\delta=0$ ) airfoil. $\eta$ was calculated at each angle of attack and for each device chord and deflection examined.

Figures $26 \mathrm{a}, 26 \mathrm{~b}$, and $26 \mathrm{c}$ give a measure of the normalized device effectiveness for the $30 \%, 40 \%$, and $50 \%$ Spoiler-Flap chords examined. Figure 26a illustrates, for a device deflection of 30 degrees, that the $50 \%$ chord device changes the suction coefficient most dramatically for angles of attack below 20 degrees.

For a device deflection of 60 degrees, Figure $26 \mathrm{~b}$ shows that the $50 \%$ and $40 \%$ chords are most effective between approximately 10 and 30 degree angles of attack. Interestingly, the shortest chord device has a greater relative impact on the suction coefficient at angles of attack above about 30 degrees.

The normalized device effectiveness for a deflection of 90 degrees is presented in Figure 26c. Again, the $50 \%$ and $40 \%$ devices are most effective over the low-to-moderate (i.e., 10 to 40 degree) angle of attack range. As was observed previously, the $30 \%$ chord device is most effective at higher angles of attack (i.e., greater than 40 degrees). 


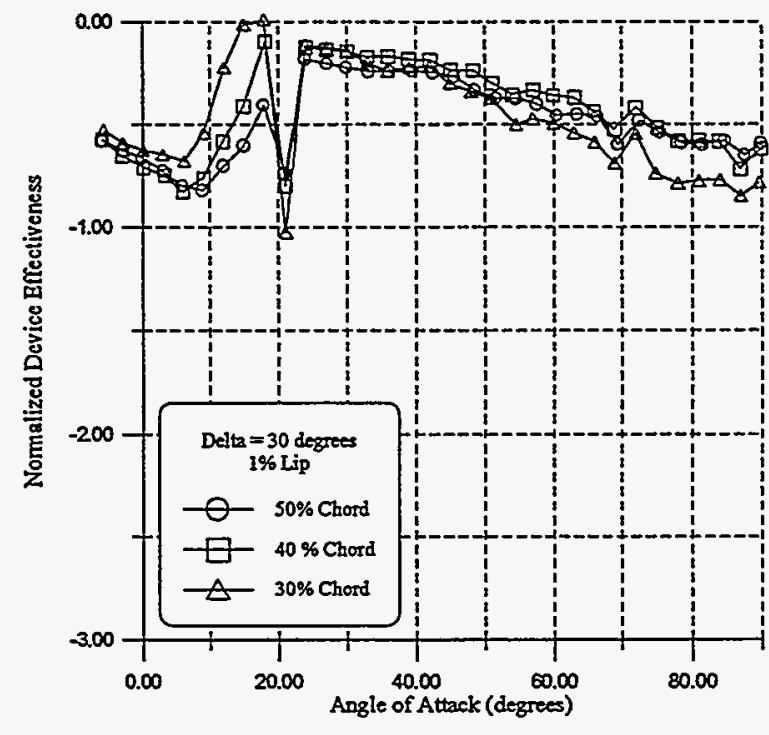

Figure 26a. Delta of $\mathbf{3 0}$ degrees

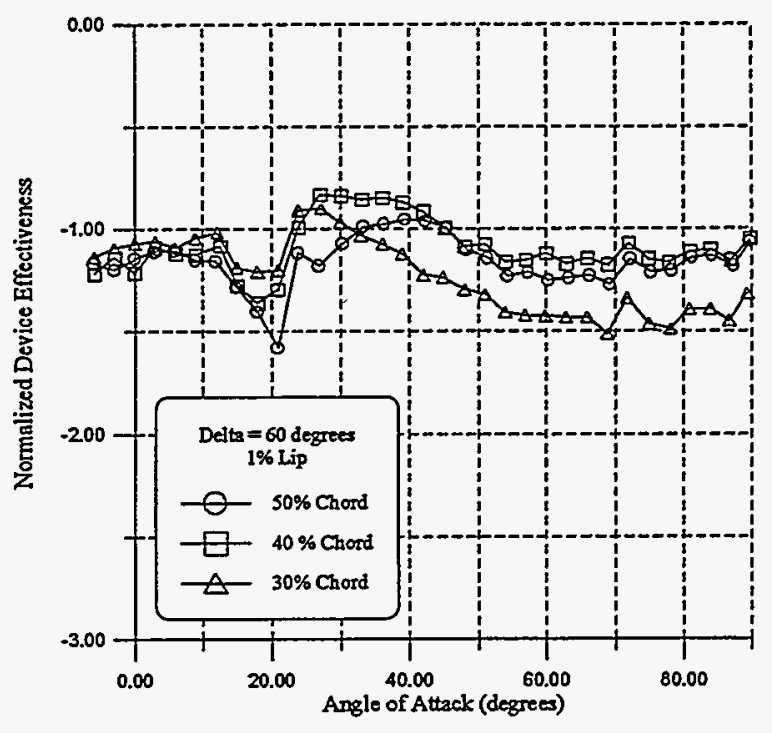

Figure 26b. Delta of 60 degrees

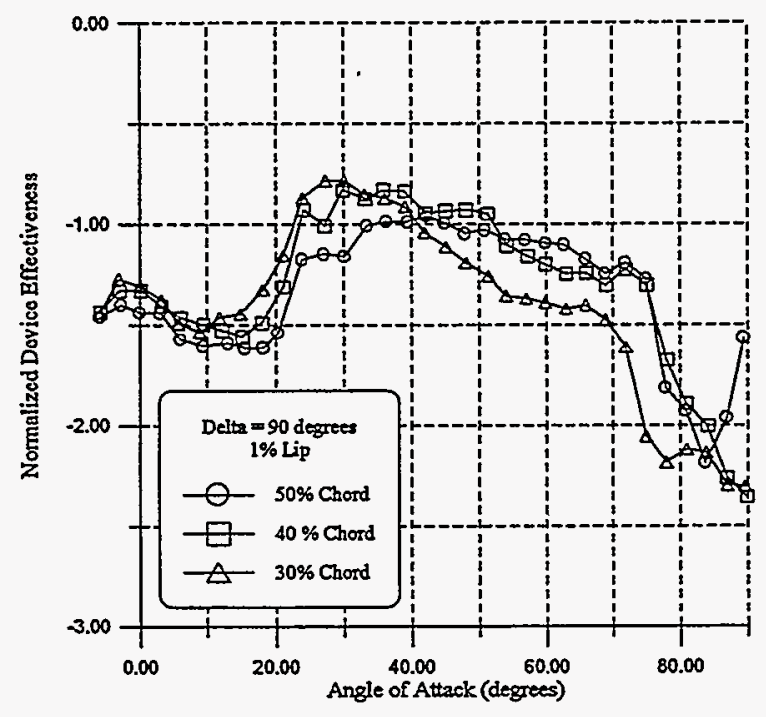

Figure 26c. Delta of 90 degrees

Figure 26. Normalized device effectiveness at three deflections 


\section{Effect of Device Chord (5\% Lip Length)}

Figures 27-29 ( $a, b$, and c) show the effect of device chord, in this case for a 5\% lip length, as a function of device deflection and angle of attack. The same basic trends, as were discussed for the $1 \%$ lip cases, are observed again. The larger chord device offers the best overall performance, and differences between the $30 \%$ and $40 \%$ chord devices are minimal for angles of attack larger than approximately 20 degrees.

A careful comparison between the $1 \%$ and $5 \%$ lip length results, as shown in Figures $23-29$, indicates that the $5 \%$ lip length has a greater impact on the $50 \%$ chord device. Unfortunately, increasing the lip length to $5 \%$ has only a minimal effect on the behavior of the shorter chord devices. 


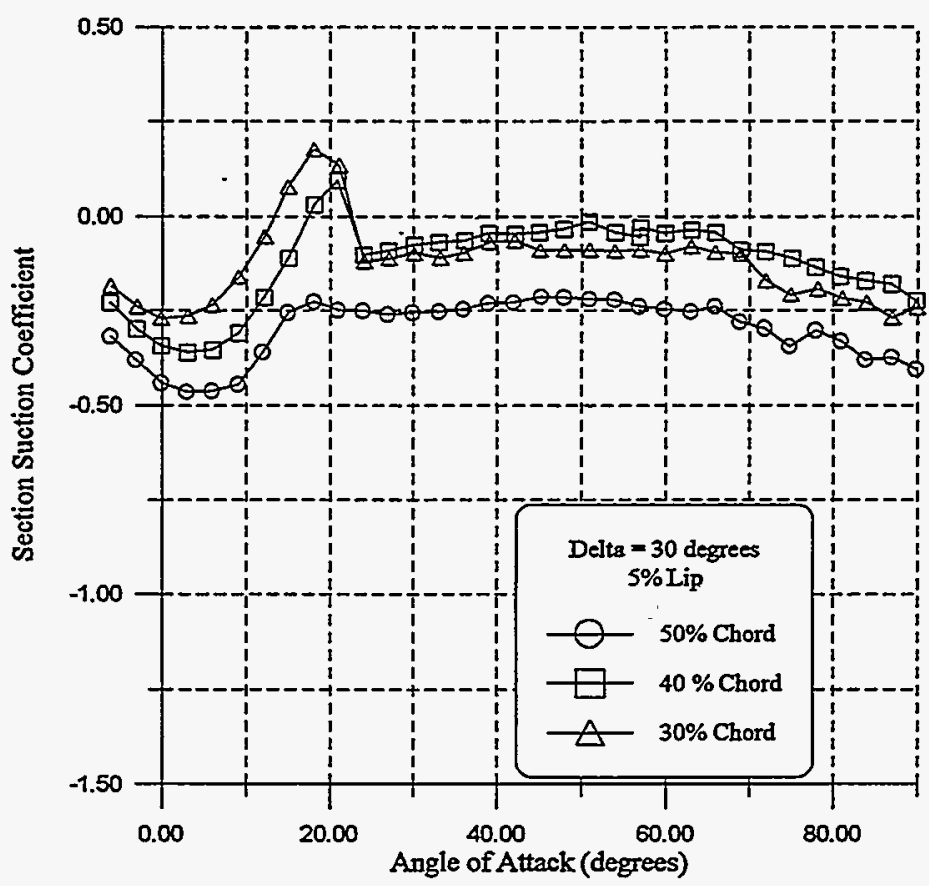

Figure 27a. Suction coefficient behavior

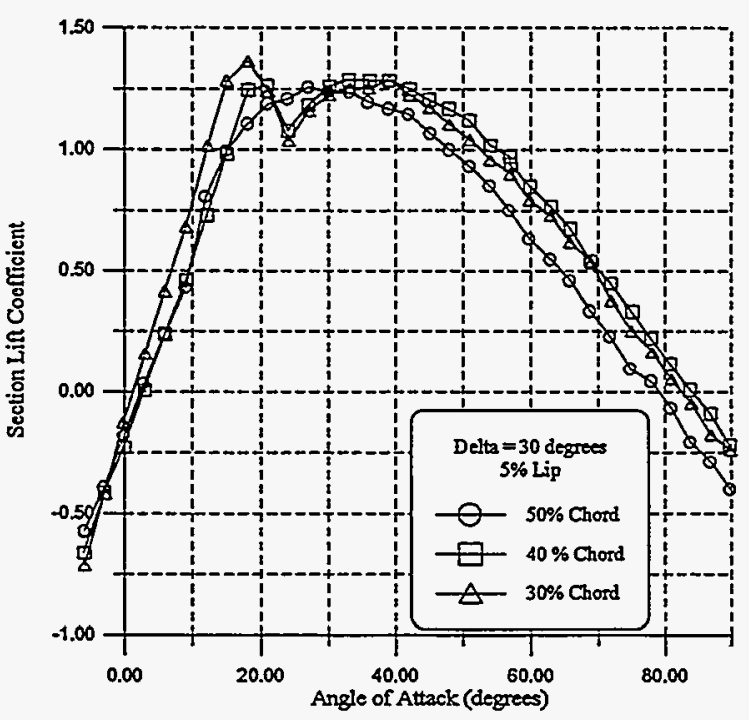

Figure 27b. Lift coefficient behavior

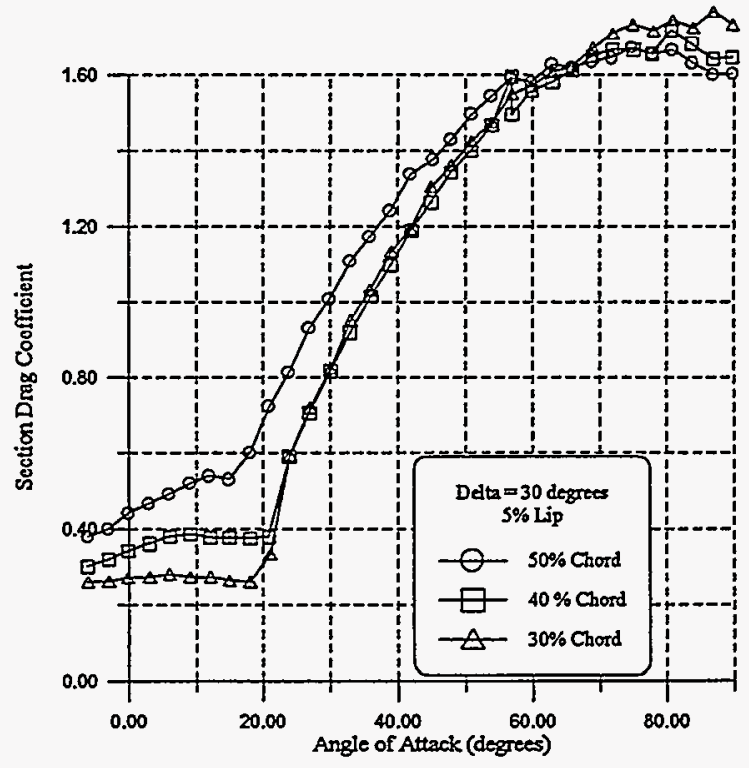

Figure 27c. Drag coefficient behavior

Figure 27. Impact of device chord with a $5 \%$ lip length at a delta of 30 degrees 


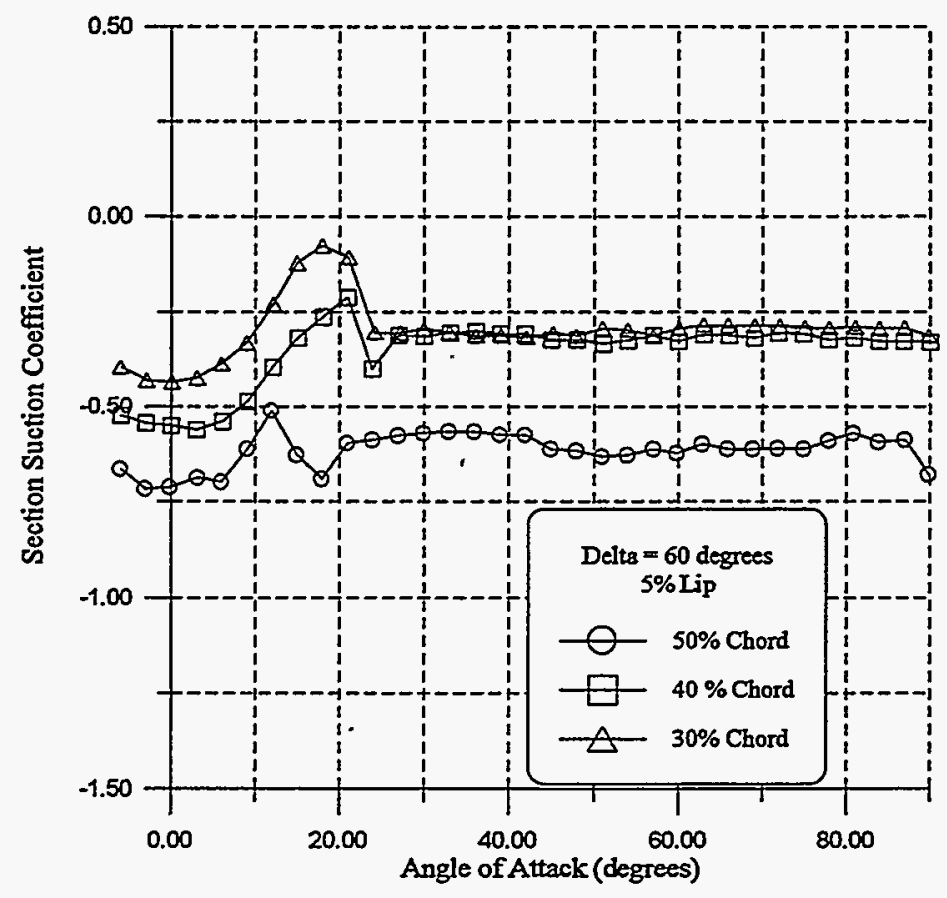

Figure 28a. Suction coefficient behavior

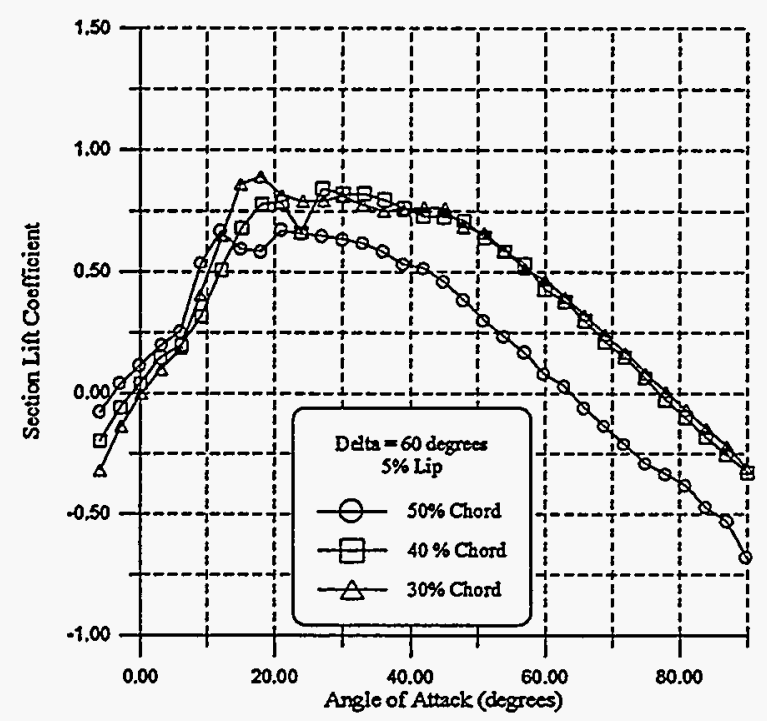

Figure 28b. Lift coefficient behavior

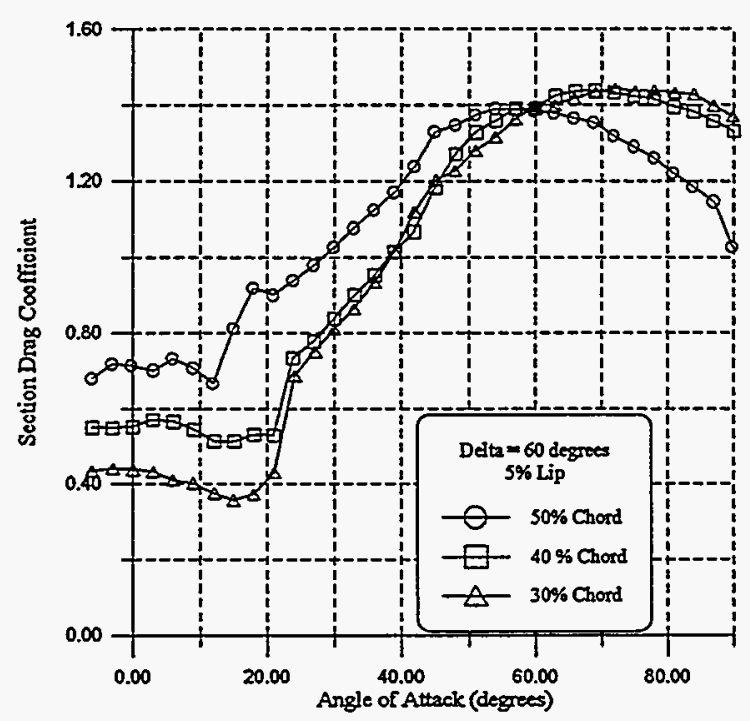

Figure 28c. Drag coefficient behavior

Figure 28. Impact of device chord with a $5 \%$ lip length at a delta of 60 degrees 


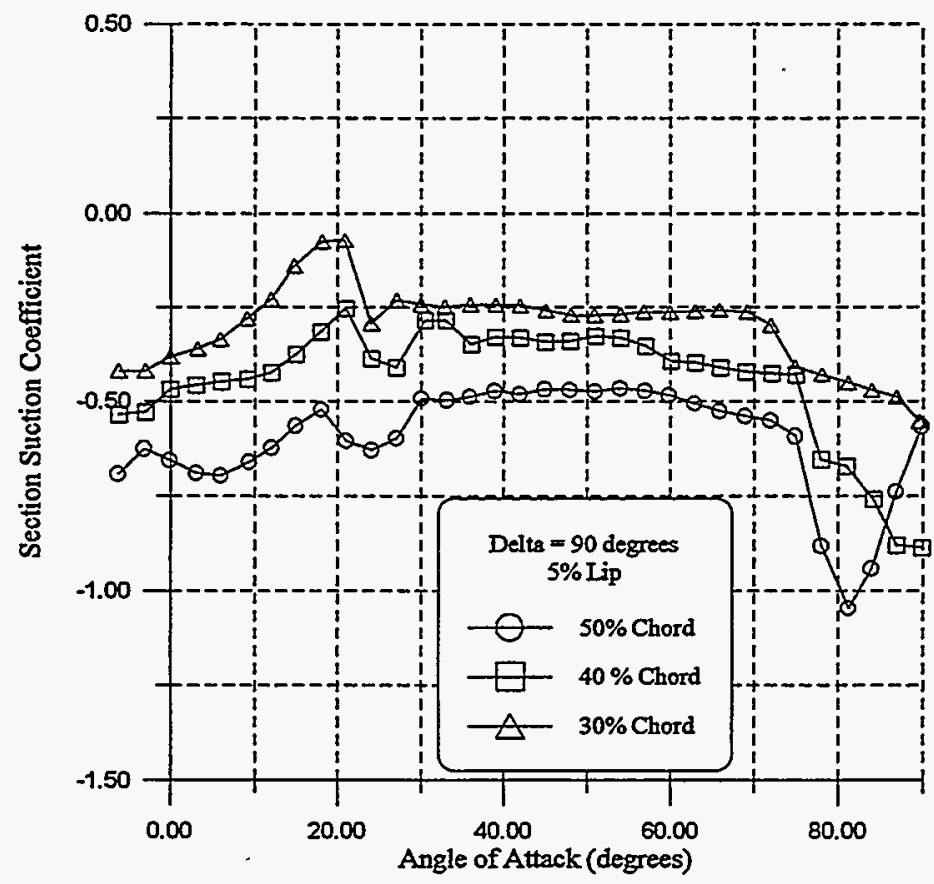

Figure 29a. . Suction coefficient behavior

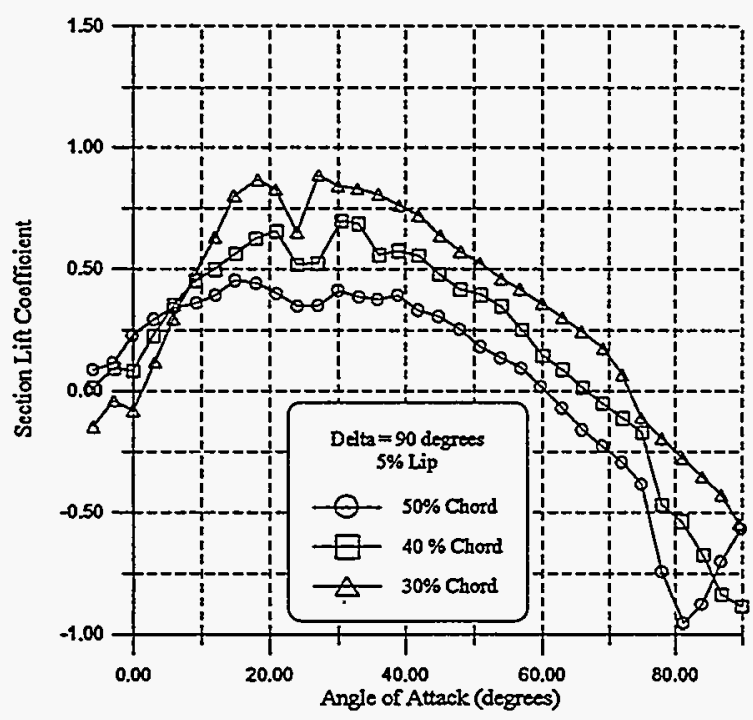

Figure 29b. Lift coefficient behavior

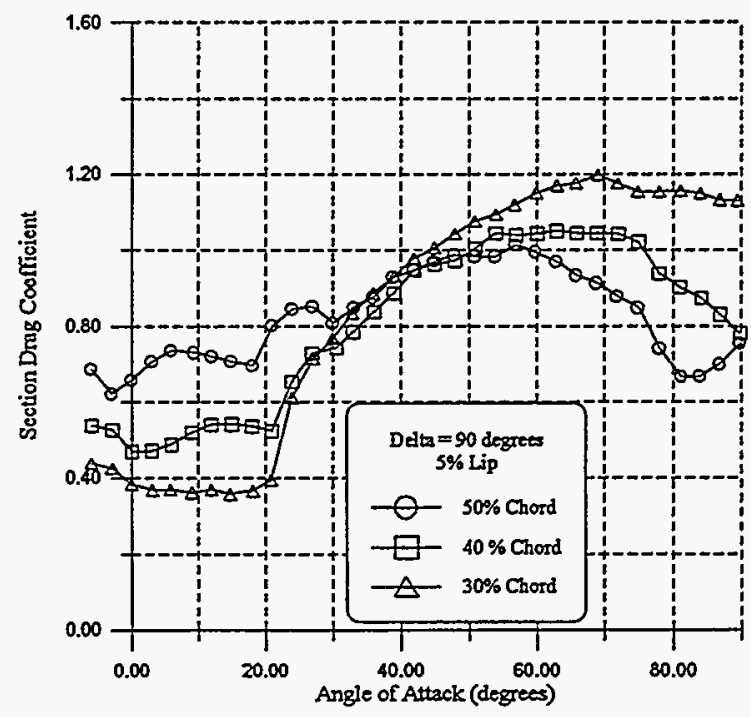

Figure 29c. Drag coefficient behavior

Figure 29. Impact of device chord with a $\mathbf{5 \%}$ lip length at a delta of $\mathbf{9 0}$ degrees 


\section{Impact of Lip on $\mathbf{4 0 \%}$ Chord Device}

The impact of lip length was examined, once more, in an effort to better identify the potential for performance enhancements on the $40 \%$ chord device. Results, presented in Figures 30-32 (a, b, and c), show the effect of lip length as a function of device deflection and angle of attack.

For device deflections of less than 60 degrees, lip length effects are most pronounced at angles of attack below 25 degrees, where a $5 \%$ lip provides a slightly better suction coefficient behavior. However, this trend reverses when the device deflection increases to 90 degrees. For this condition, the shorter $1 \%$ lip offers slightly better performance.

It is important to note for the three device deflections examined, differences in the suction coefficient are relatively minor at angles of attack above approximately 25 degrees. 


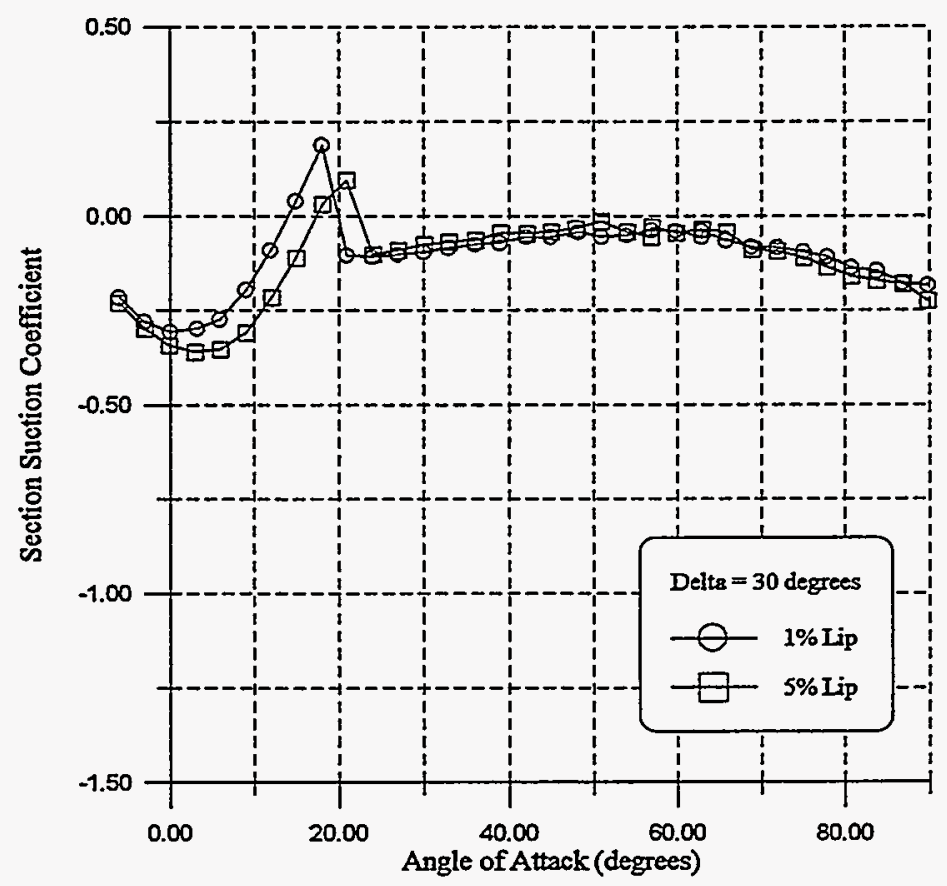

Figure 30a. Suction coefficient behavior

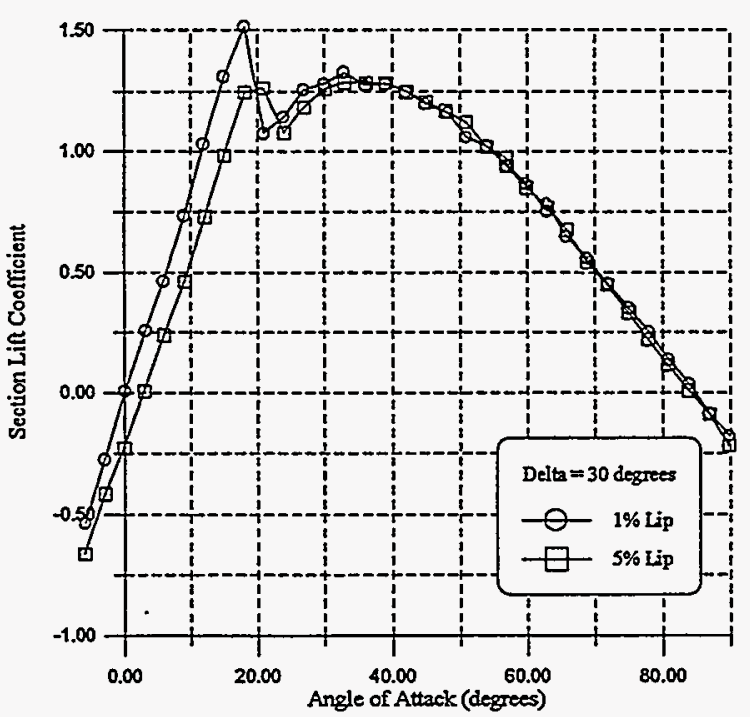

Figure 30b. Lift coefficient behavior

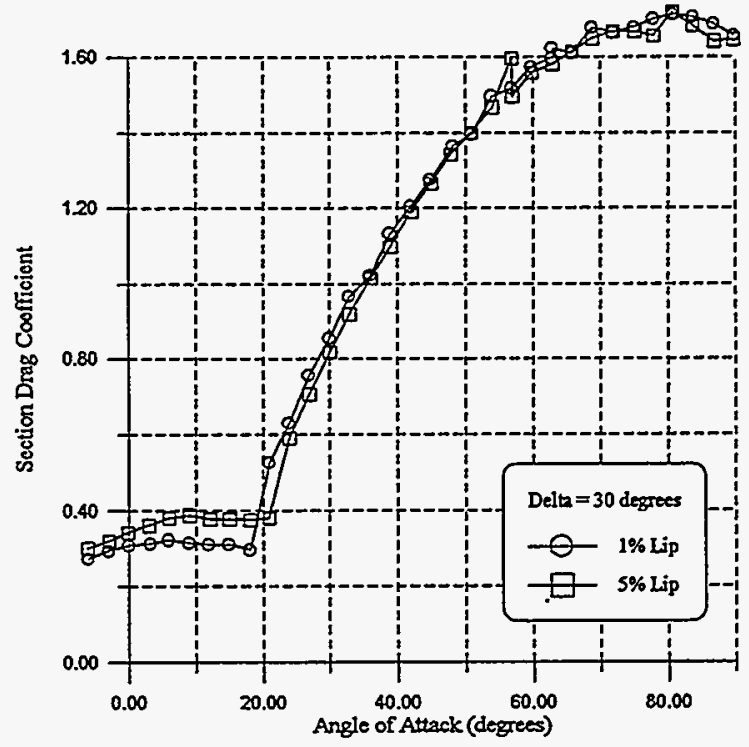

Figure 30c. Drag coefficient behavior

Figure 30. Impact of lip length on a $\mathbf{4 0 \%}$ chord device at a delta of $\mathbf{3 0}$ degrees 


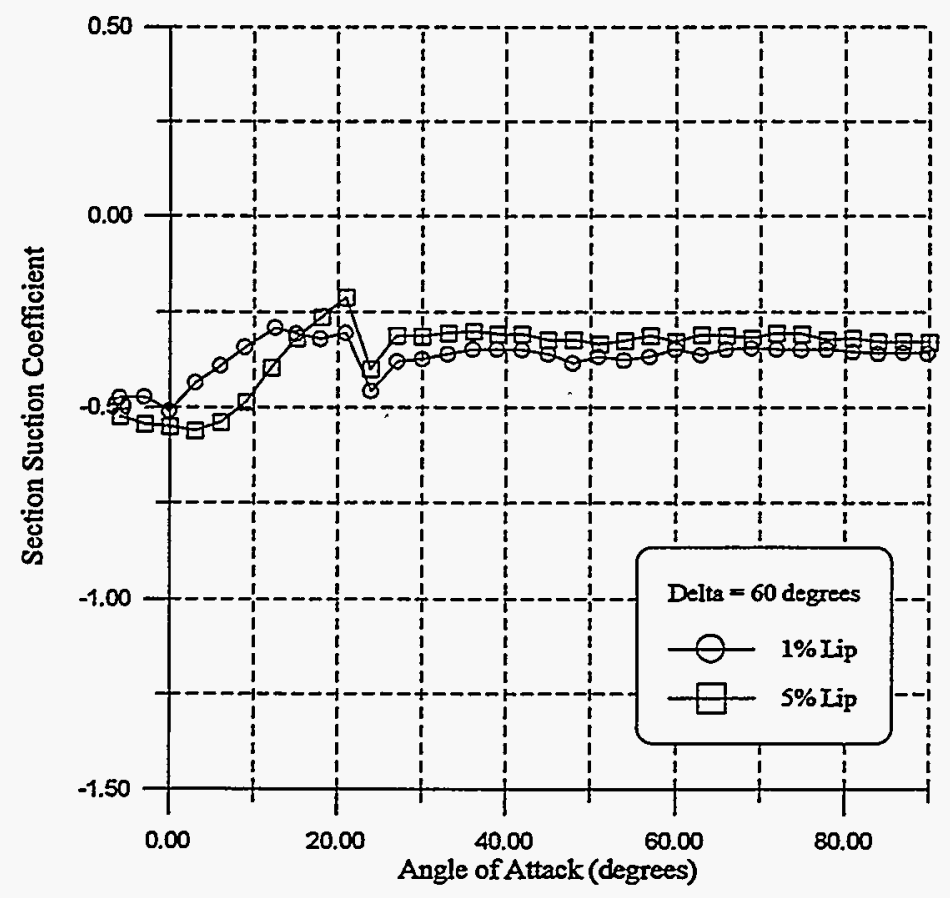

Figure 31a. Suction coefficient behavior

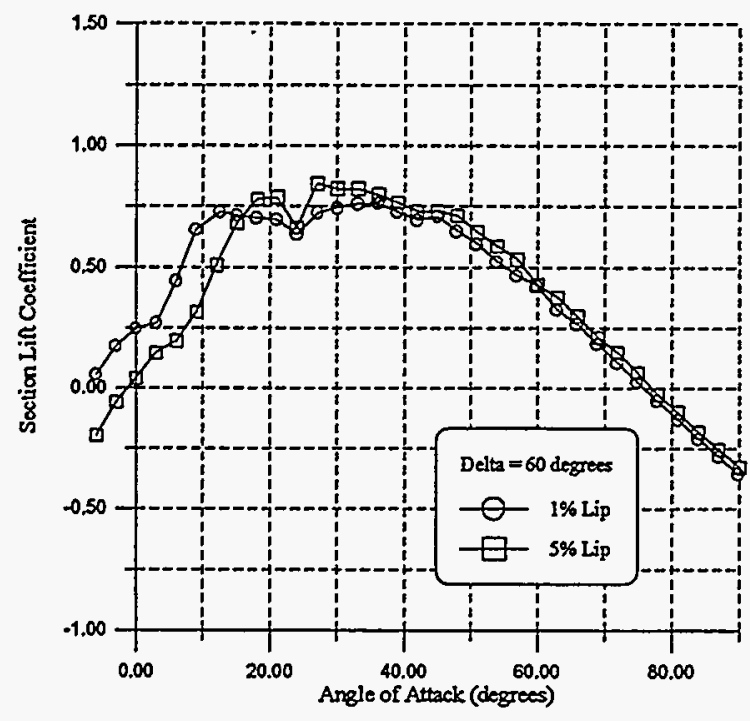

Figure 31b. Lift coefficient behavior

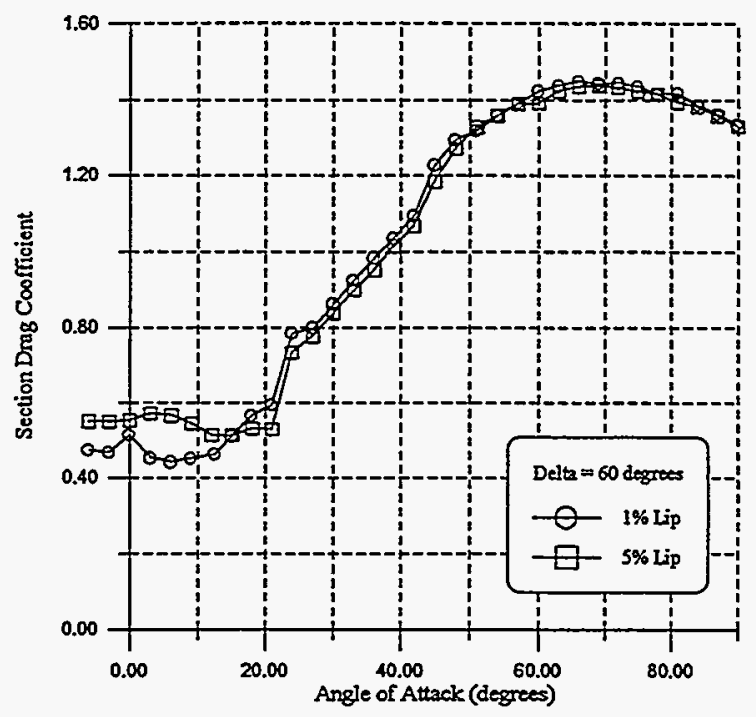

Figure 31c. Drag coefficient behavior

Figure 31. Impact of lip length on a $40 \%$ chord device at a delta of 60 degrees 


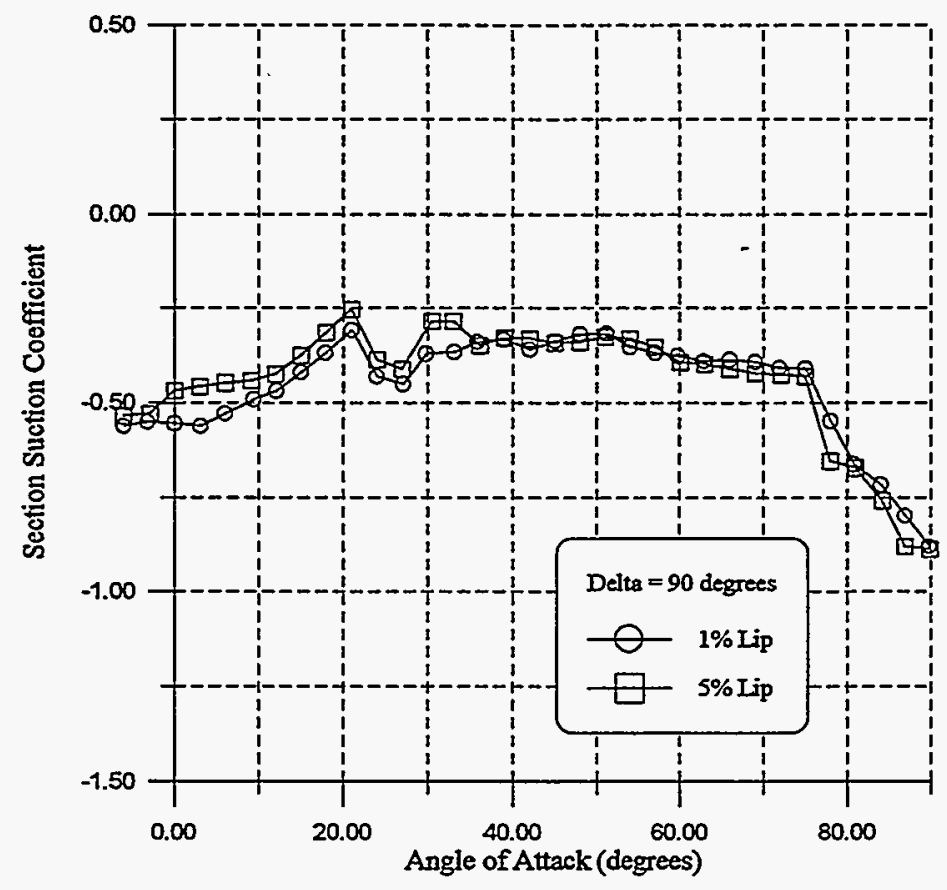

Figure 32a. Suction coefficient behavior

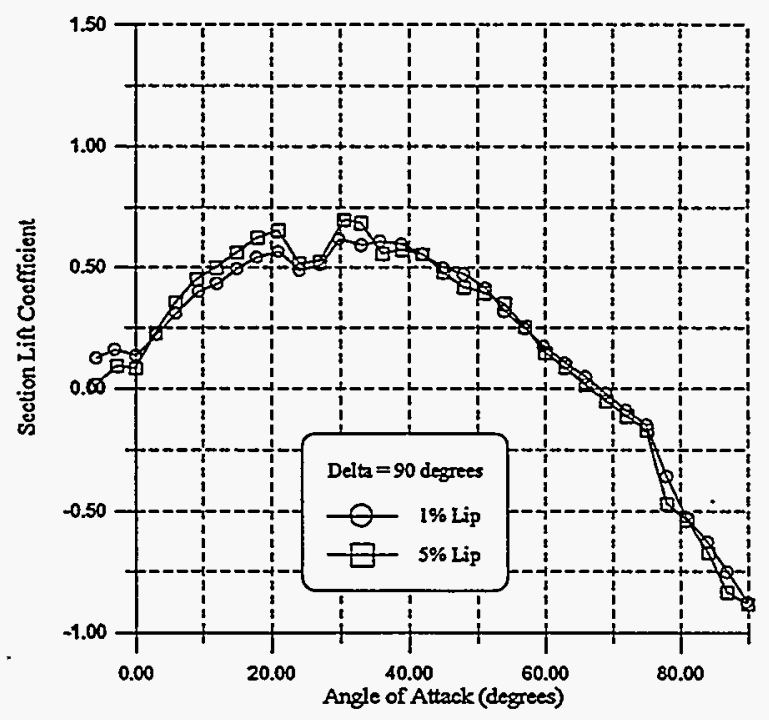

Figure 32b. Lift coefficient behavior

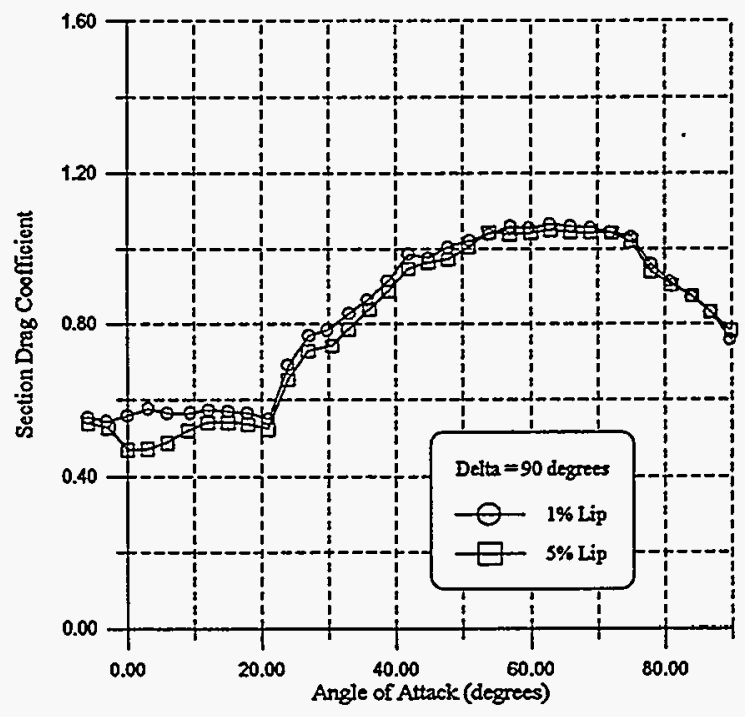

Figure 32c. Drag coefficient behavior

Figure 32. Impact of lip length on a $40 \%$ chord device at a delta of 90 degrees 


\section{Impact of Lip on $30 \%$ Chord Device}

The impact of lip length was further examined, in this case to identify the potential for performance enhancements on the $30 \%$ chord device. Results, presented in Figures 33-35 (a, b, and c), show the effect of lip length as a function of device deflection and angle of attack.

The results for the $30 \%$ device are very similar to those observed for the $40 \%$ chord device and are most apparent at angles of attack less than 25 degrees. The $5 \%$ lip length offers slightly better performance for device deflections of less than 60 degrees. Interestingly, the shorter lip provides a more negative suction coefficient behavior when the device is fully opened to the 90 degree position.

It is important to note that in virtually all of the cases examined, and shown in Figures 30-35, lip length effects are relatively small in comparison to those observed for device chord changes. 


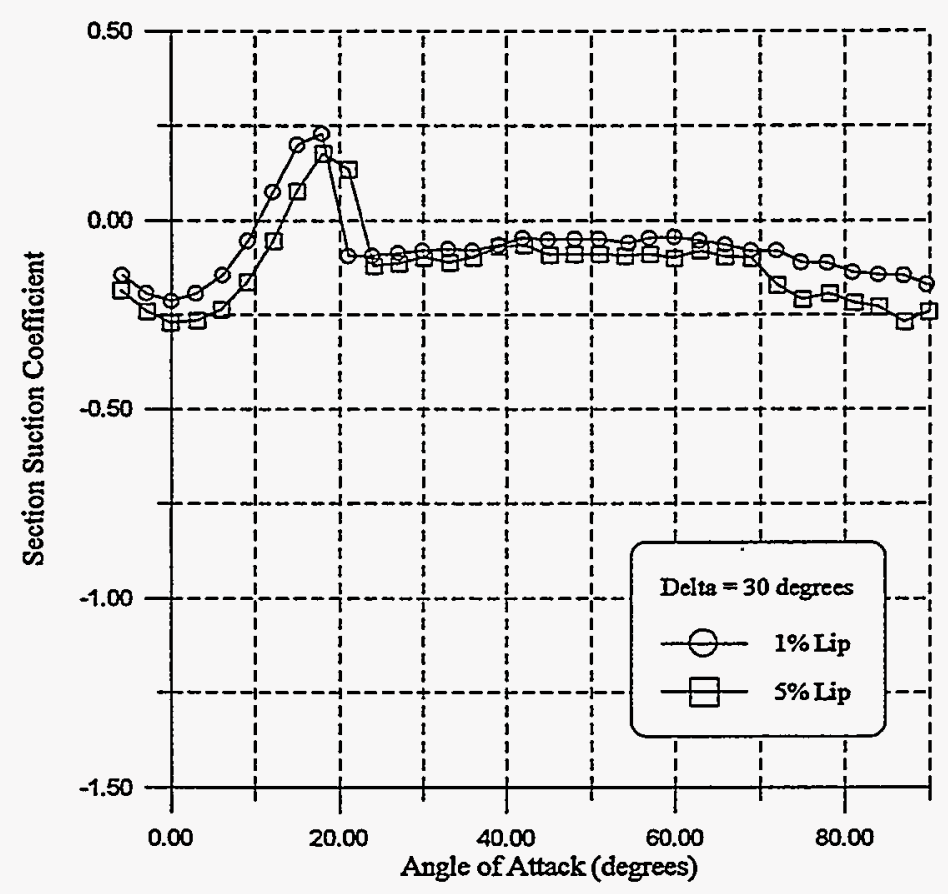

Figure 33a. Suction coefficient behavior

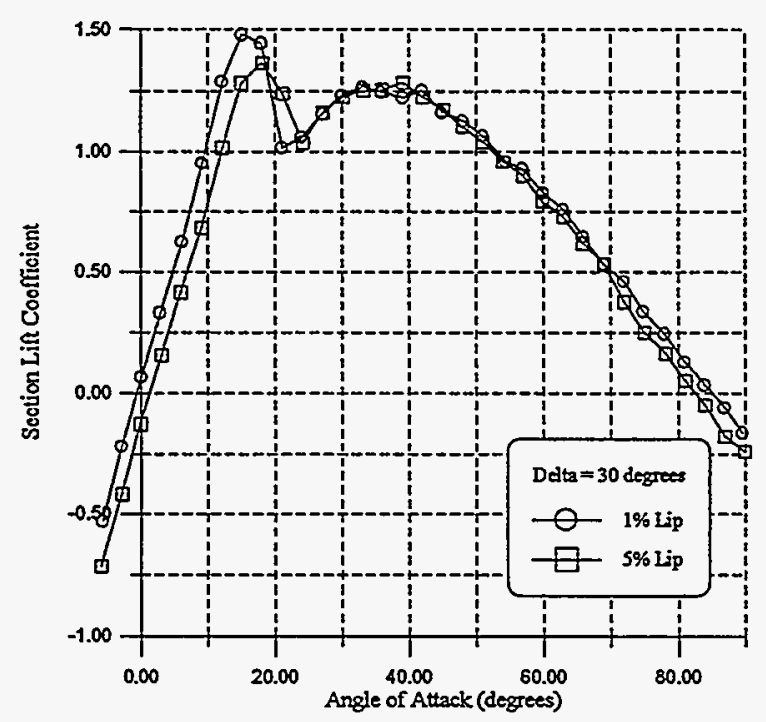

Figure 33b. Lift coefficient behavior

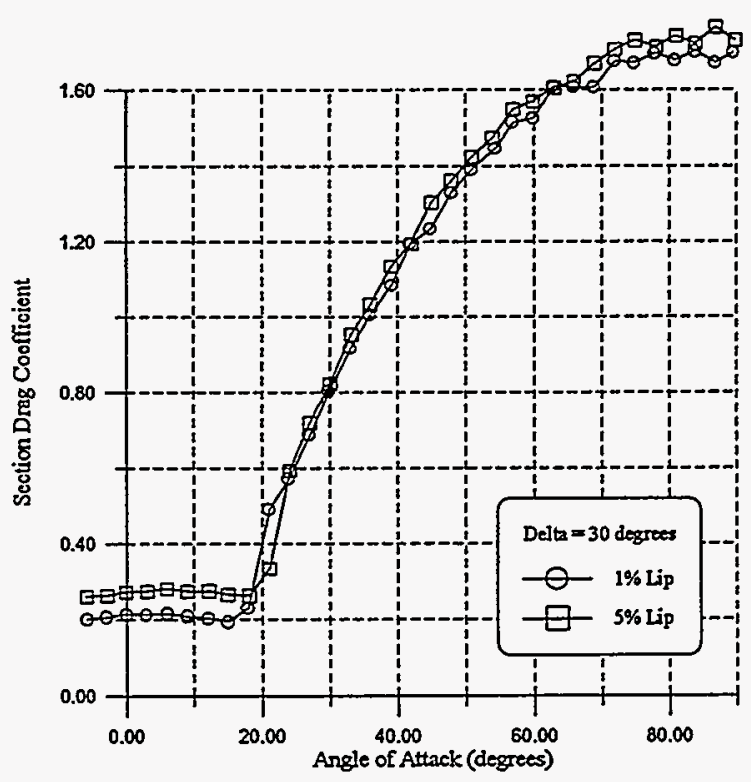

Figure 33c. Drag coefficient behavior

Figure 33. Impact of lip length on a $\mathbf{3 0 \%}$ chord device at a delta of $\mathbf{3 0}$ degrees 


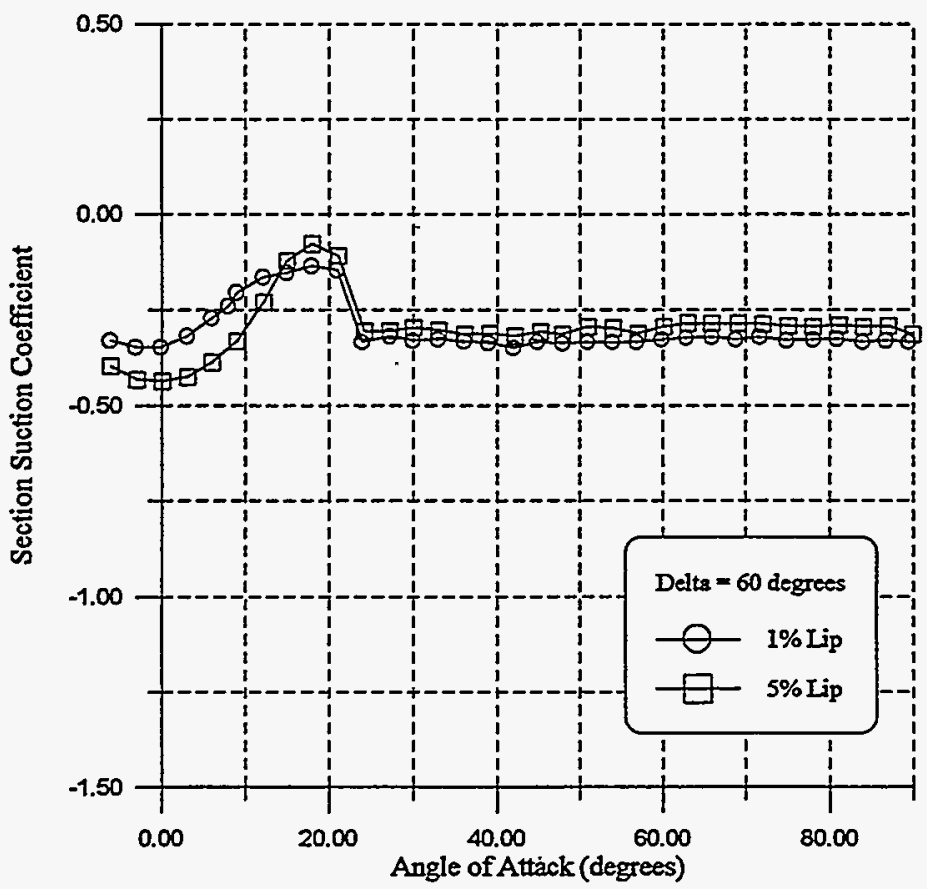

Figure 34a. Suction coefficient behavior

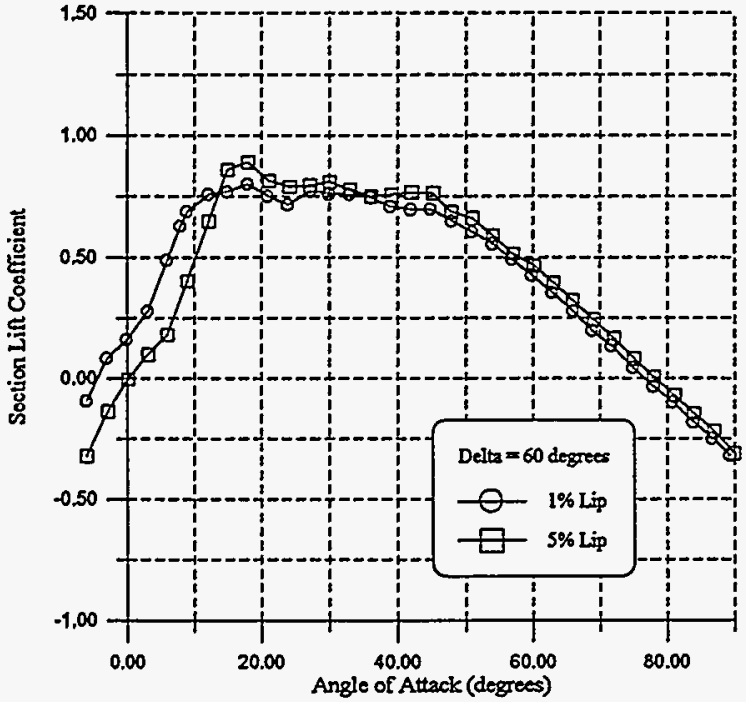

Figure 34b. Lift coefficient behavior

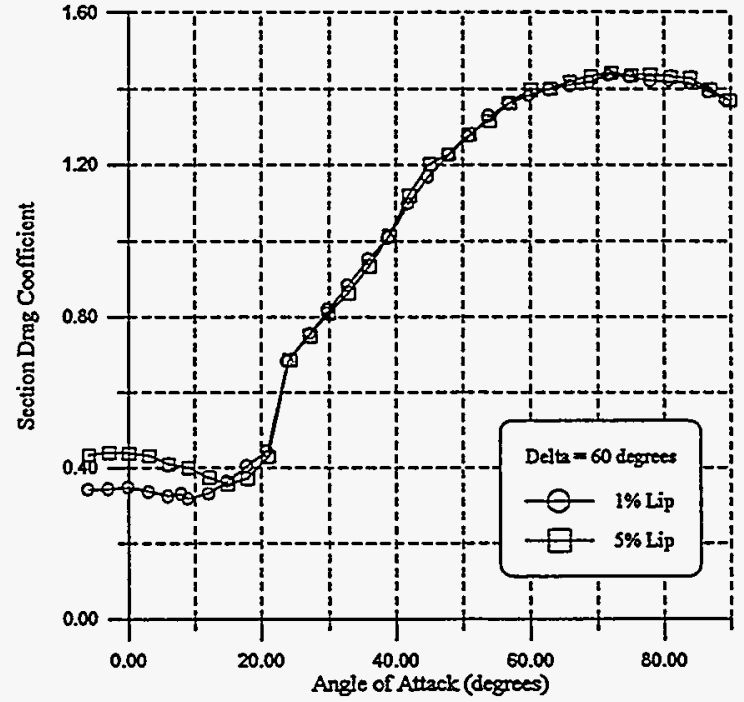

Figure 34c. Drag coefficient behavior

Figure 34. Impact of lip length on a $30 \%$ chord device at a delta of 60 degrees 


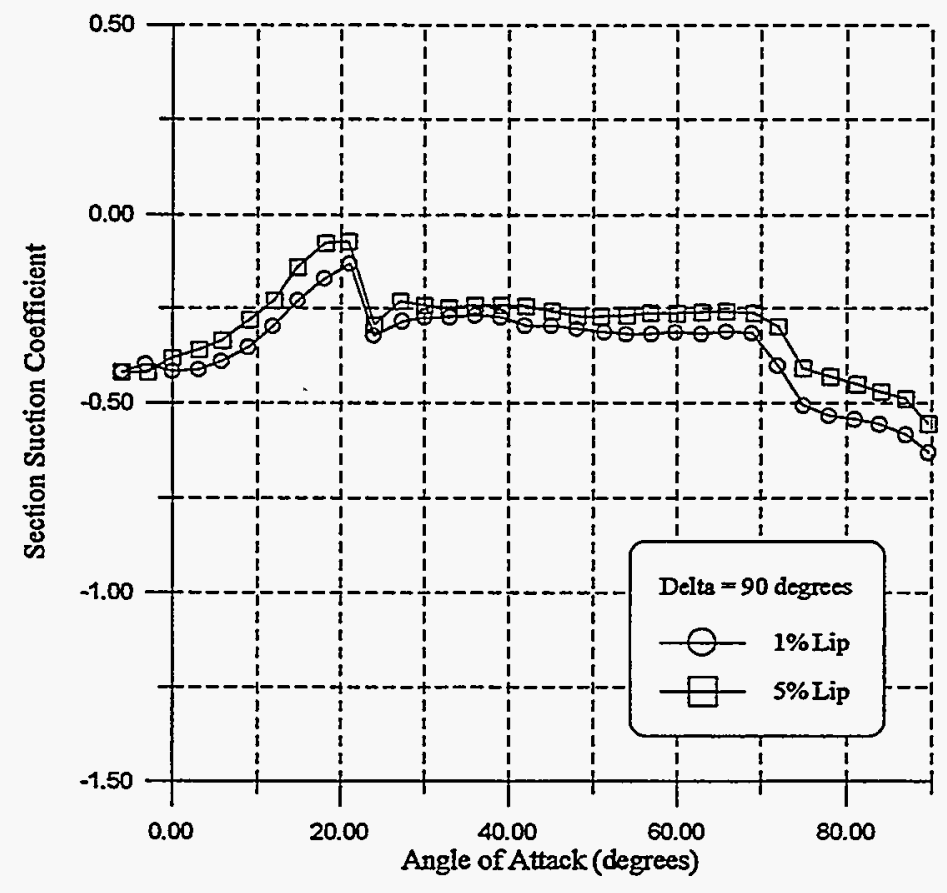

Figure 35a. Suction coefficient behavior

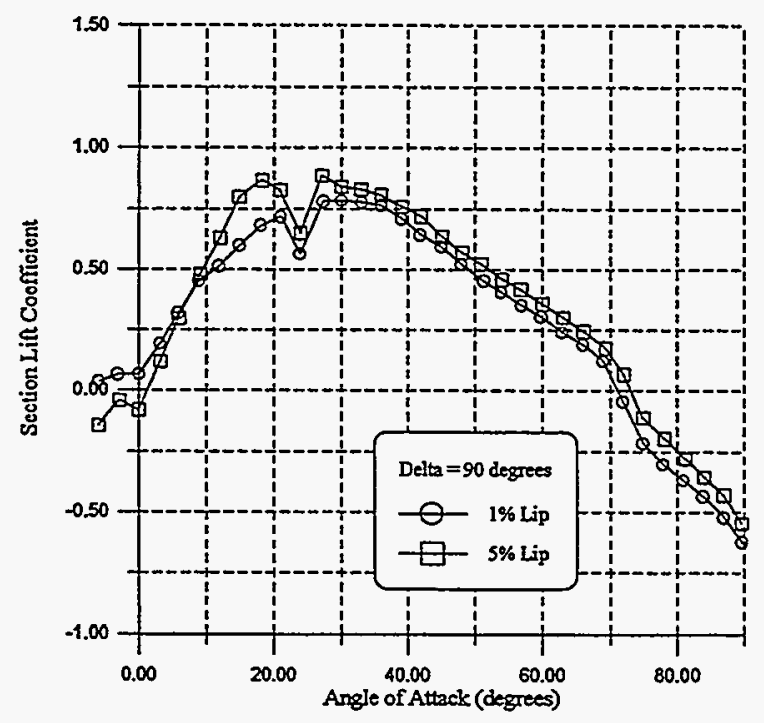

Figure 35b. Lift coefficient behavior

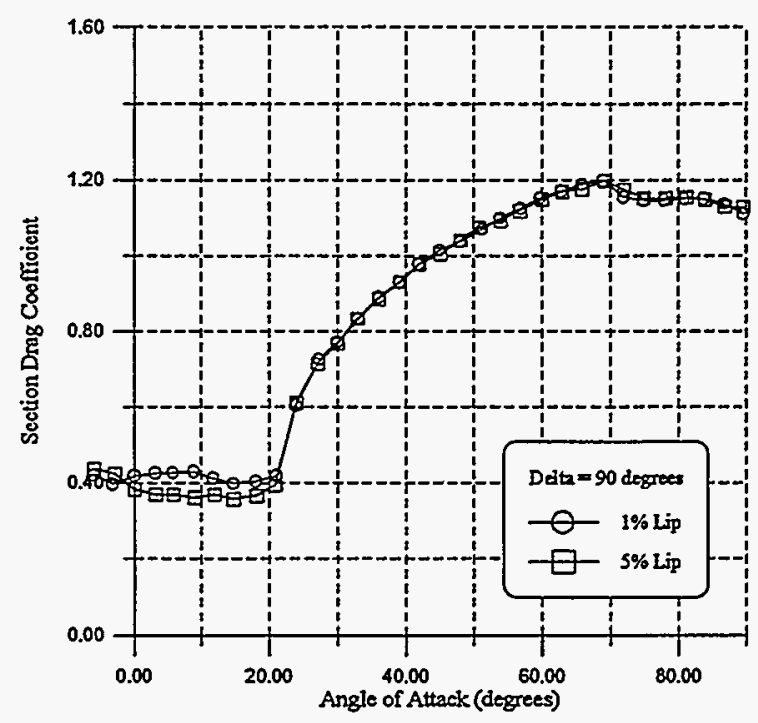

Figure 35c. Drag coefficient behavior

Figure 35. Impact of lip length on a $\mathbf{3 0 \%}$ chord device at a delta of 90 degrees 


\section{Effect of Pivot Location on the $\mathbf{5 0 \%}$ Chord Device}

Figures $36 a, b$, and $c$ show the effect on aerodynamic performance of moving the $50 \%$ chord device pivot point $10 \%$ aft, from the normal Pivot 3 location, to Pivot 4 . Figure 9 shows the exact location of each pivot point.

Because a shorter chord device was preferred, results for only one device deflection of 10 degrees were obtained. As Figure 36a shows, the effect of moving the pivot location aft is minimal and restricted to a small angle of attack range, between about 20 and 28 degrees. Additional testing would be necessary to further define the effect of pivot location for the $50 \%$ chord device. 


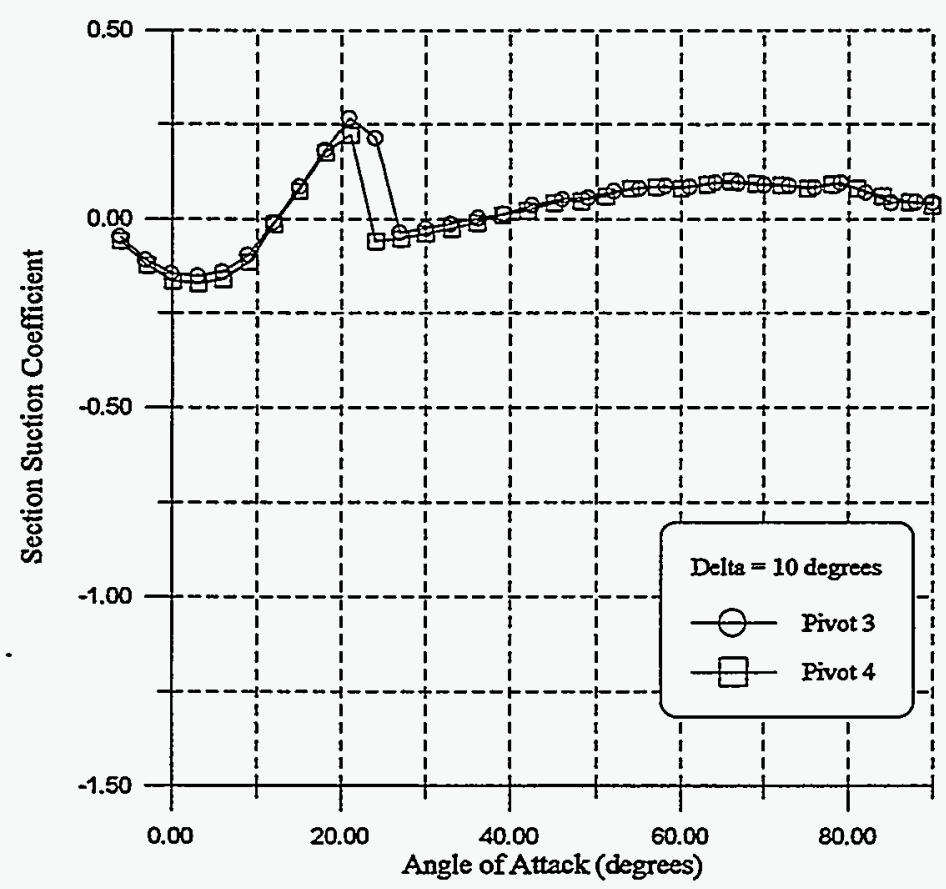

Figure 36a. Suction coefficient behavior

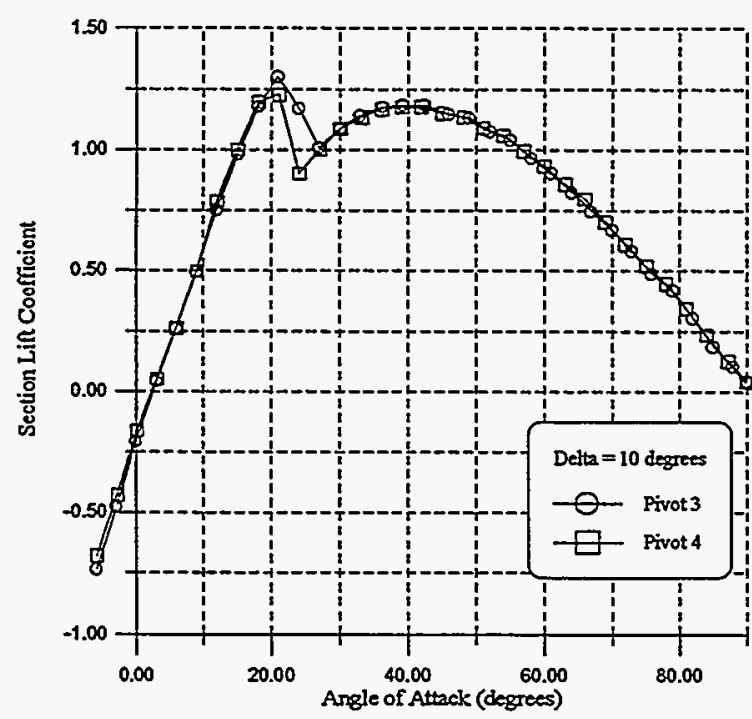

Figure 36b. Lift coefficient behavior

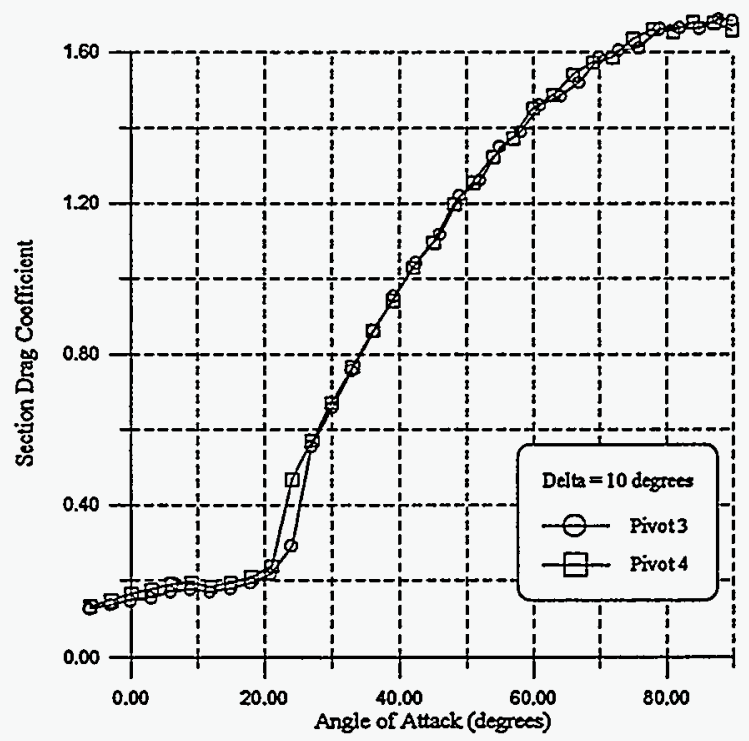

Figure 36c. Drag coefficient behavior

Figure 36. Impact of pivot location for a $\mathbf{5 0 \%}$ chord device at a delta of $\mathbf{1 0}$ degrees Effect of Pivot Location on the $\mathbf{4 0 \%}$ Chord Device 
Figures 37 and $38(a, b$, and $c)$ illustrate the effect of moving the $40 \%$ chord device pivot point, from Pivot 4 , to Pivot 5 (10\% aft) and Pivot 6 (5\% forward and 2.5\% down). Again, see Figure 9 for more information on the exact pivot locations. Results only for device deflections of 60 and 90 degrees were obtained.

Figures $37 \mathrm{a}$ and $38 \mathrm{a}$ show that device pivot location has only a minor effect on the $40 \%$ device's suction coefficient behavior, except at angles of attack greater than 40 degrees. Specific trends and differences are difficult to identify, thus suggesting that the aerodynamic performance of the device is relatively insensitive to pivot location. This particular observation is significant, because a pivot point offering a more reasonable hinge moment behavior could be selected, without concern for a significant loss in suction coefficient performance. 


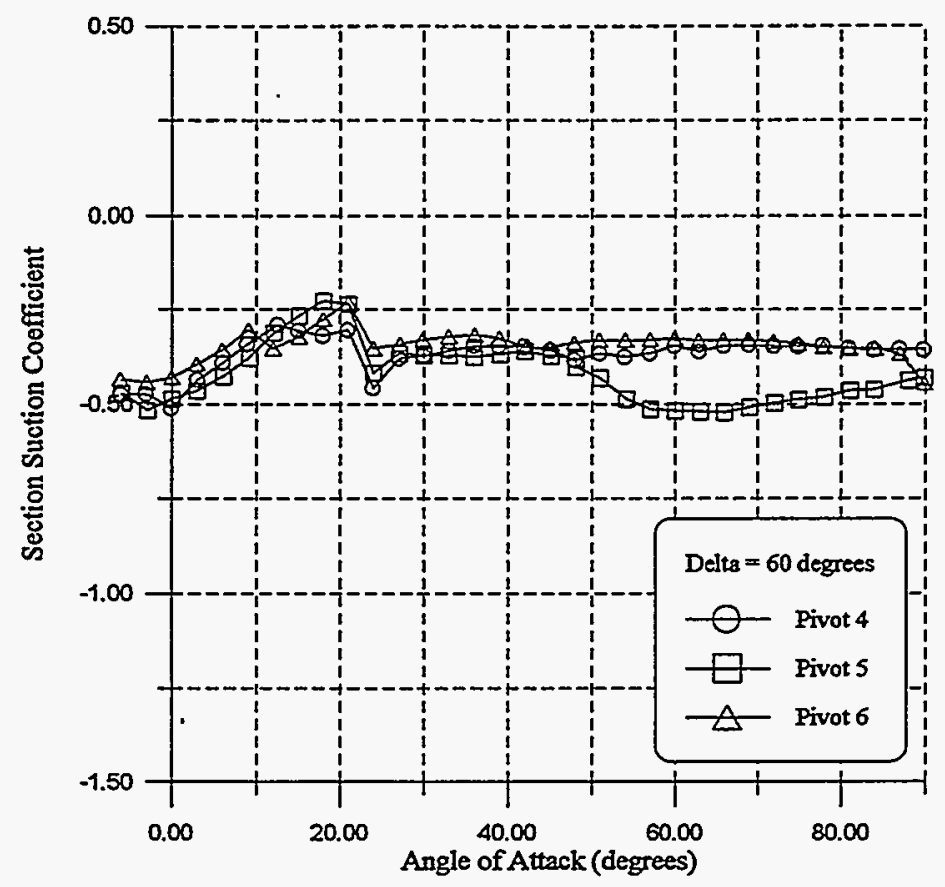

Figure 37a. Suction coefficient behavior

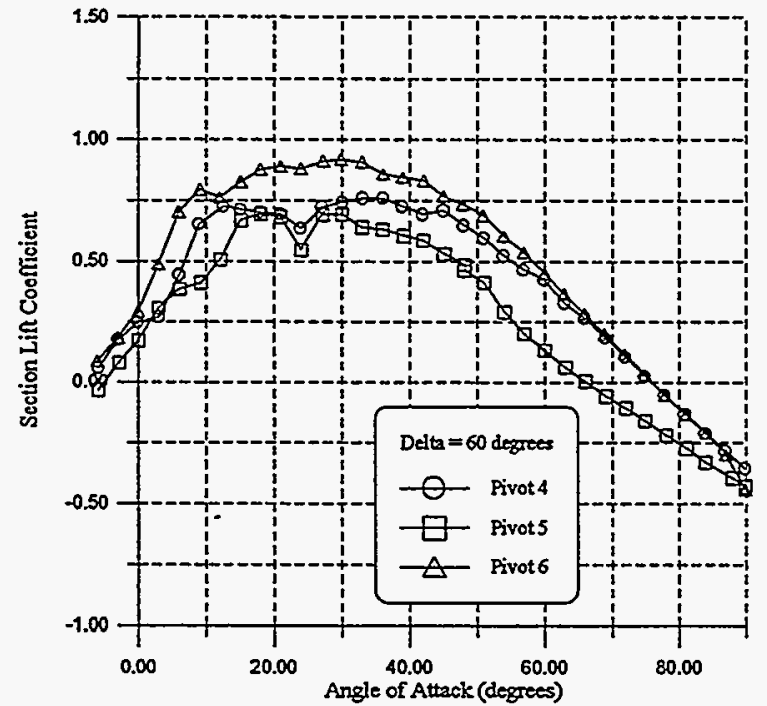

Figure 37b. Lift coefficient behavior

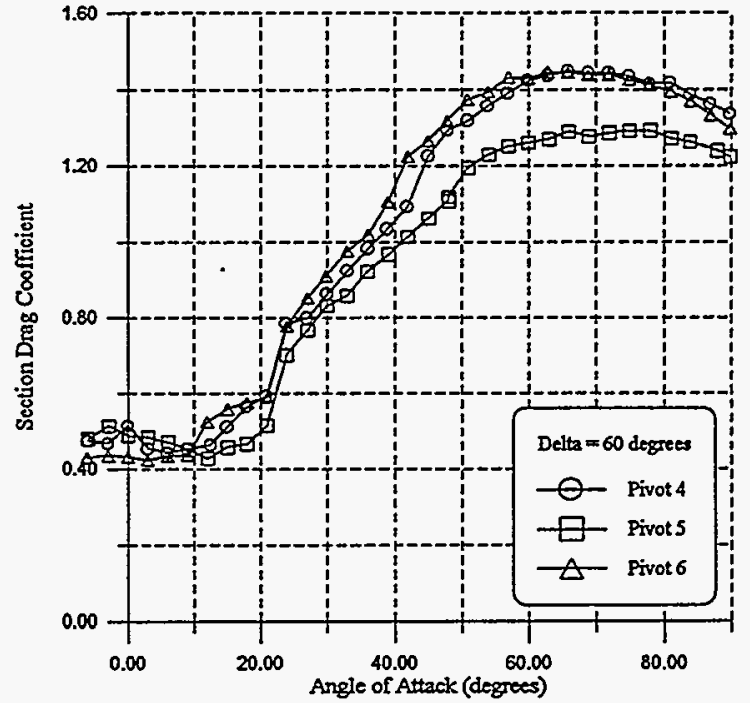

Figure 37c. Drag coefficient behavior

Figure 37. Impact of pivot location for a $40 \%$ chord device at a delta of 60 degrees 


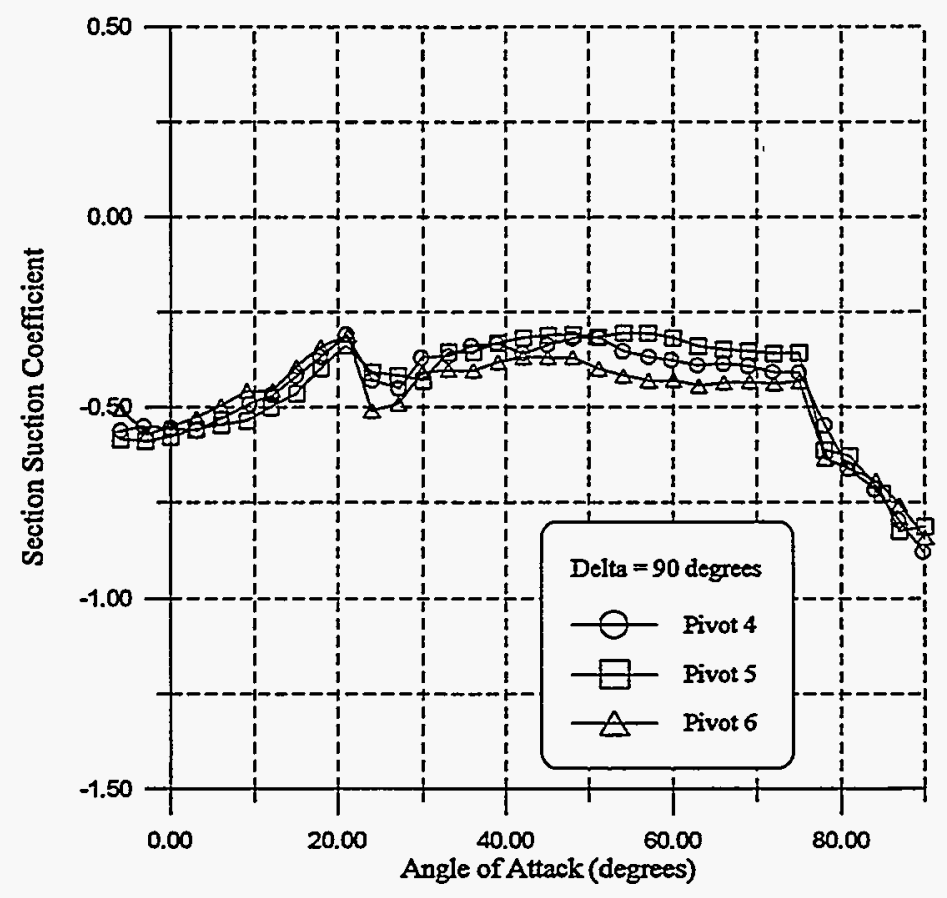

Figure 38a. Suction coefficient behavior

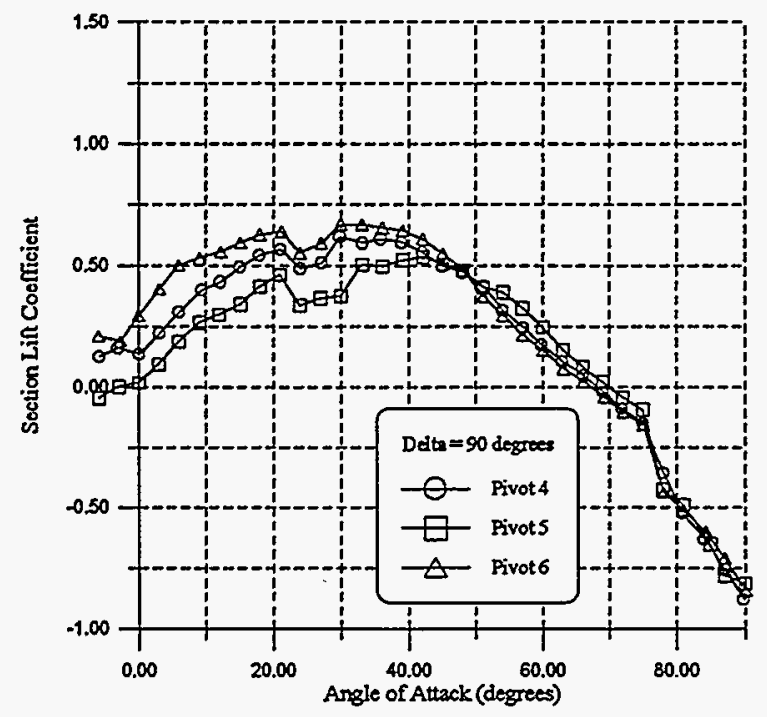

Figure 38b. Lift coefficient behavior

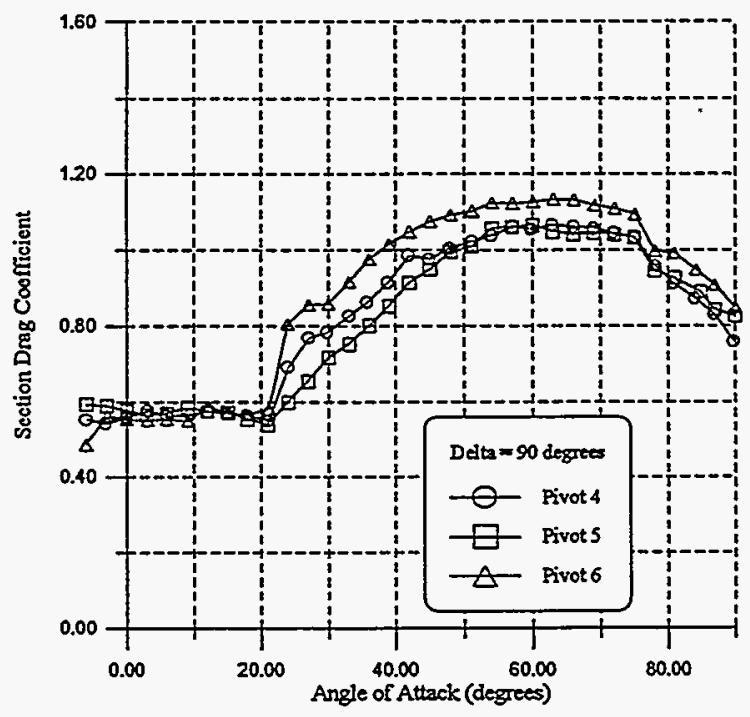

Figure 38c. Drag coefficient behavior

Figure 38. Impact of pivot location for a $\mathbf{4 0} \%$ chord device at a delta of $\mathbf{9 0}$ degrees 


\section{Hinge Moments}

Figures 39 through 42 examine the hinge moment behavior of various Spoiler-Flap configurations as a function of device deflection and angle of attack. The results are expressed in terms of a device hinge moment coefficient $\left(\mathrm{Cm}_{\mathfrak{h}}\right)$, defined as follows:

$$
\mathrm{Cm}_{\mathrm{h}}=\left(\mathrm{M}_{\mathrm{c}}\right) /(\mathrm{q})\left(\mathrm{c}_{\mathrm{c}}{ }^{2}\right)
$$

where $M_{c}$ is the device hinge moment (per unit span), $q$ is the local dynamic pressure, and $c_{c}$ is the device chord length. A positive moment coefficient indicates a clockwise (i.e., trailing-edge down) torque is applied to the device.

Results surveying the impact of lip length, vent angle, device chord and pivot location are provided on the following pages. 


\section{Effect of Lip Length}

Figure 39 identifies the effect of lip length on the hinge moment behavior of the $50 \%$ chord device, as a function of device deflection and angle of attack. The $1 \%$ and $5 \%$ lip length data are represented by, respectively, solid and dashed lines.

The hinge moment coefficient behavior is nearly constant for a device deflection of 30 degrees; however, it is small in magnitude (approximately $+/-0.04$ ). As Figure 39 illustrates, for angles of attack below approximately 40 degrees, the device tends to open slightly easier with the longer (5\%) lip. Overall the impact of lip length on the hinge moment coefficient is minor, in this case.

The hinge moment coefficient magnitude, as a function of angle of attack, for device deflections of 60 and 90 degrees is predominately negative. With only one exception, Figure 39 shows that use of a longer lip tends to make the hinge moment coefficient even more negative (i.e., it makes it harder to keep the device open). For a 60 degree device deflection, the 5\% lip increases the hinge moment (i.e., it becomes more positive) for angles of attack between -6 and 12 degrees.

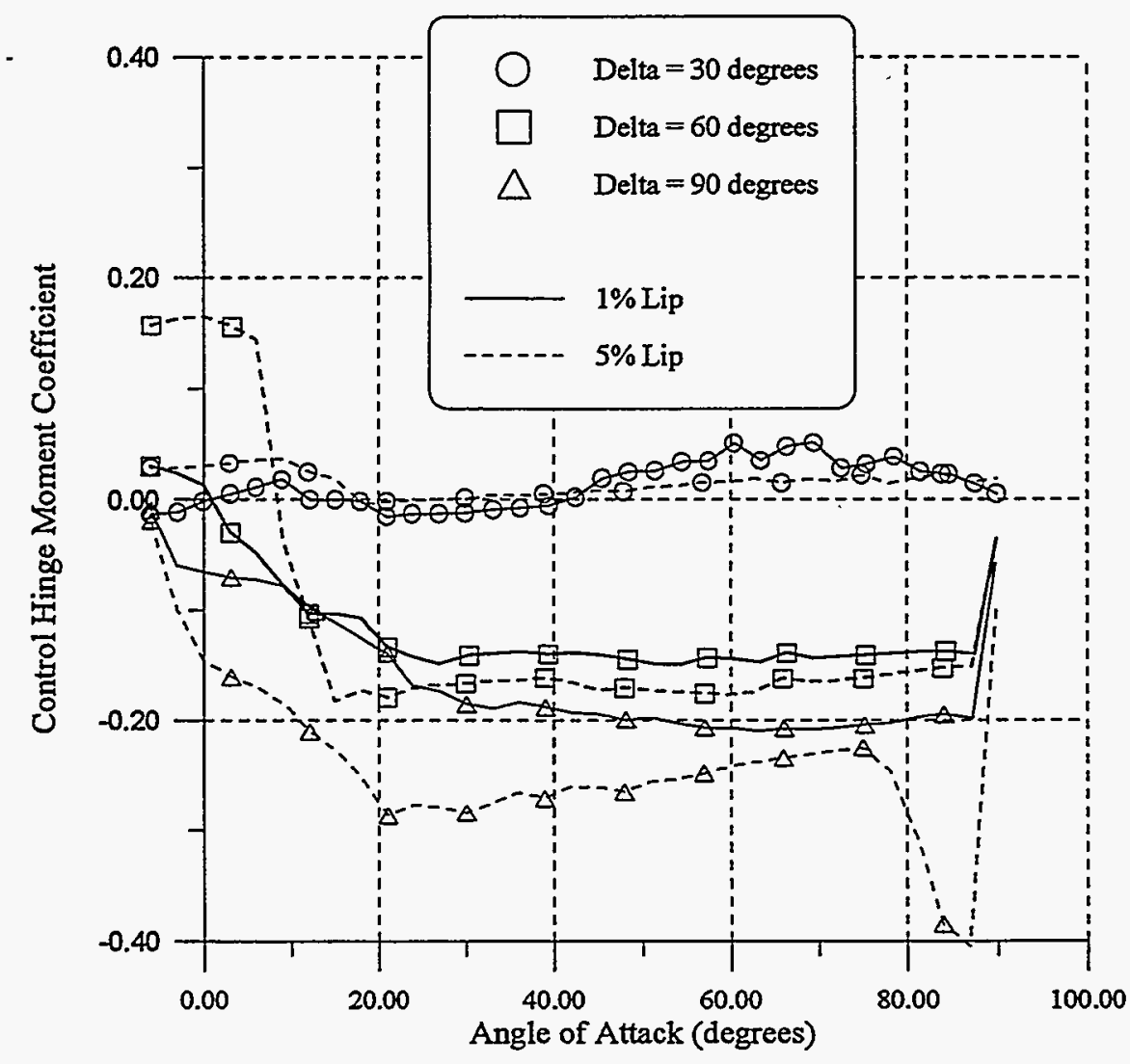

Figure 39. Impact of lip length on $\mathbf{5 0 \%}$ chord device hinge moment 


\section{Effect of Vent Angle}

Figure 40 identifies the effect of vent angle on the hinge moment behavior of the $50 \%$ chord device, as a function of device deflection and angle of attack. The 40 and 0 degree vent data is represented by, respectively, solid and dashed lines.

Results, shown in Figure 40, indicate that immediate (i.e., 0 degree) venting makes the device hinge moment coefficients significantly more negative and thus additional actuation torque is required to keep the device open, throughout the entire angle of attack range.

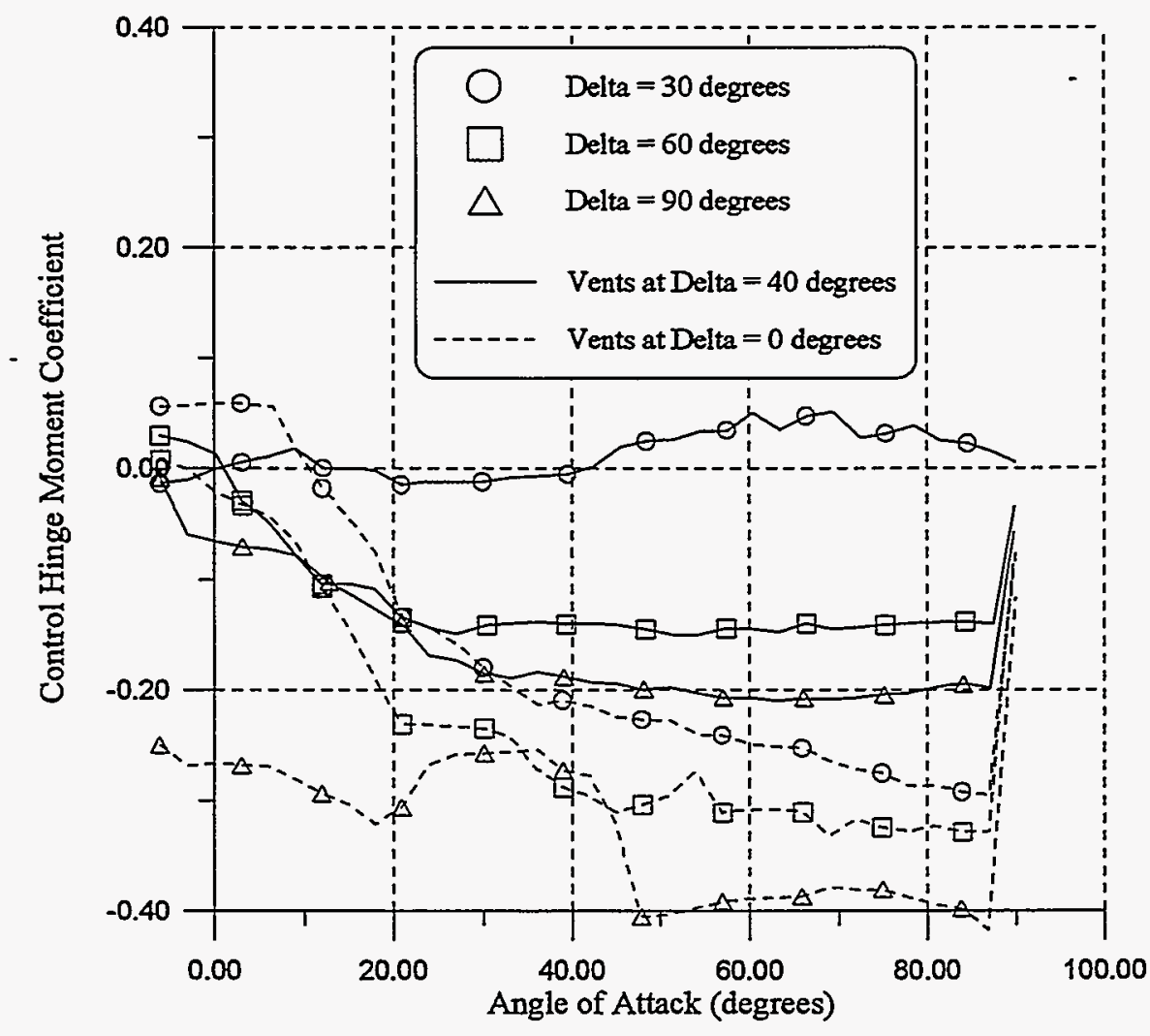

Figure 40. Impact of vent angle on $\mathbf{5 0 \%}$ chord device hinge moment 


\section{Effect of Device Chord}

Figure 41 identifies the effect of device chord on the hinge moment behavior, as a function of device deflection and angle of attack. Data for $40 \%$ and $50 \%$ chords are shown using, respectively, solid and dashed lines.

Interestingly, differences in the hinge moment behavior are most pronounced for a 30 degree device deflection where the $40 \%$ chord device has a more positive moment. This effect, however, weakens as the device is deflected to 60 or 90 degrees, where only minor moment differences due to the chord length change are observed.

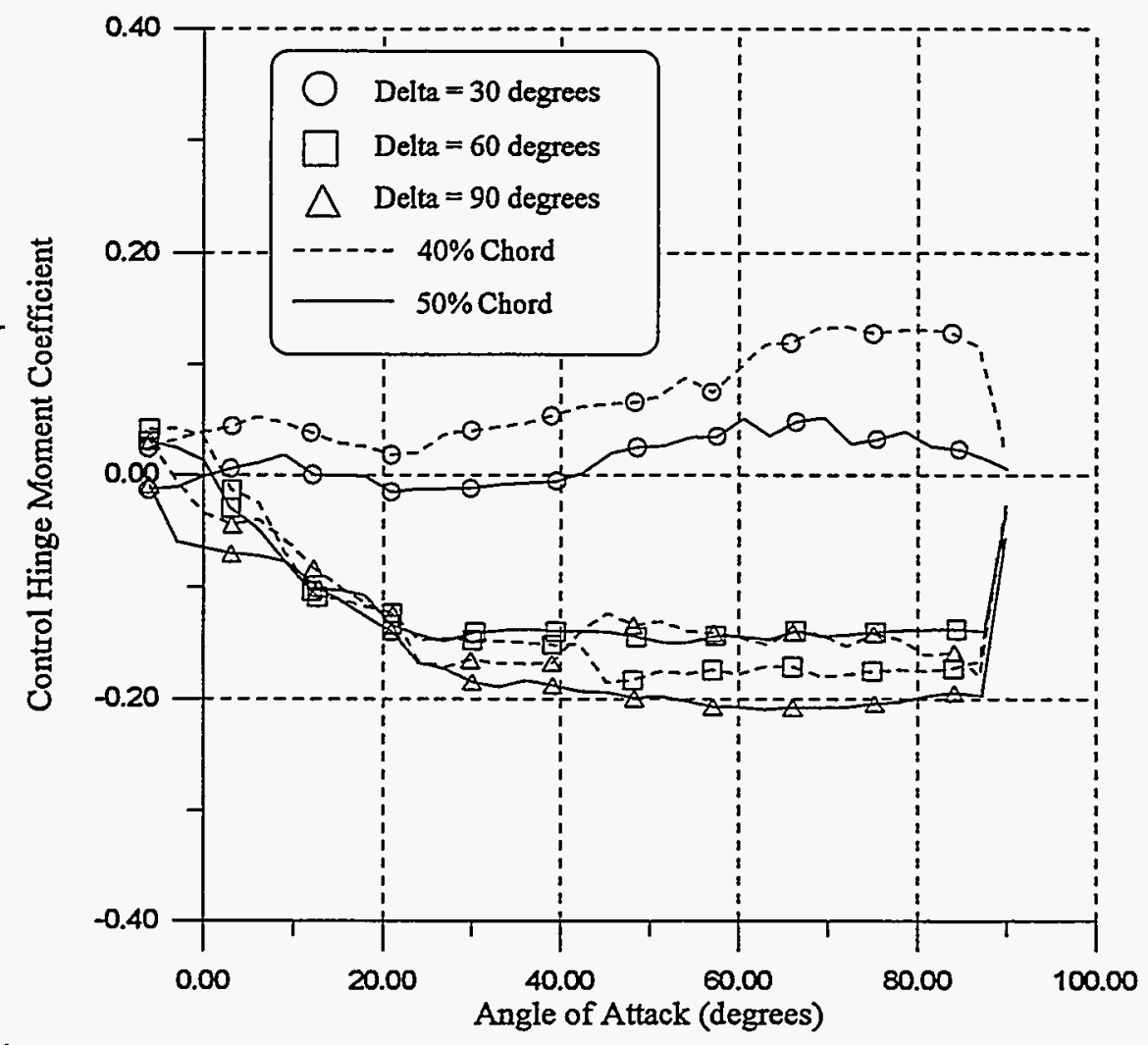

Figure 41. Impact of chord on device hinge moment 


\section{Effect of Pivot Location (40\% Chord Device)}

Figure 42 identifies the effect of pivot location on the hinge moment behavior of the $40 \%$ chord device, as a function of device deflection and angle of attack. Data for pivots 4 and 5 are shown using, respectively, solid and dashed lines. Results for device deflections of $0,30,60$, and 90 degrees are presented.

Aft movement of the device pivot point has a favorable impact on the hinge moment coefficient for each device deflection examined. As Figure 42 shows, use of pivot 5 results in a moment coefficient change of at least positive 0.10 The difference is dramatic and for all device deflections, except 90 degrees, the coefficients become positive over the entire angle of attack range.

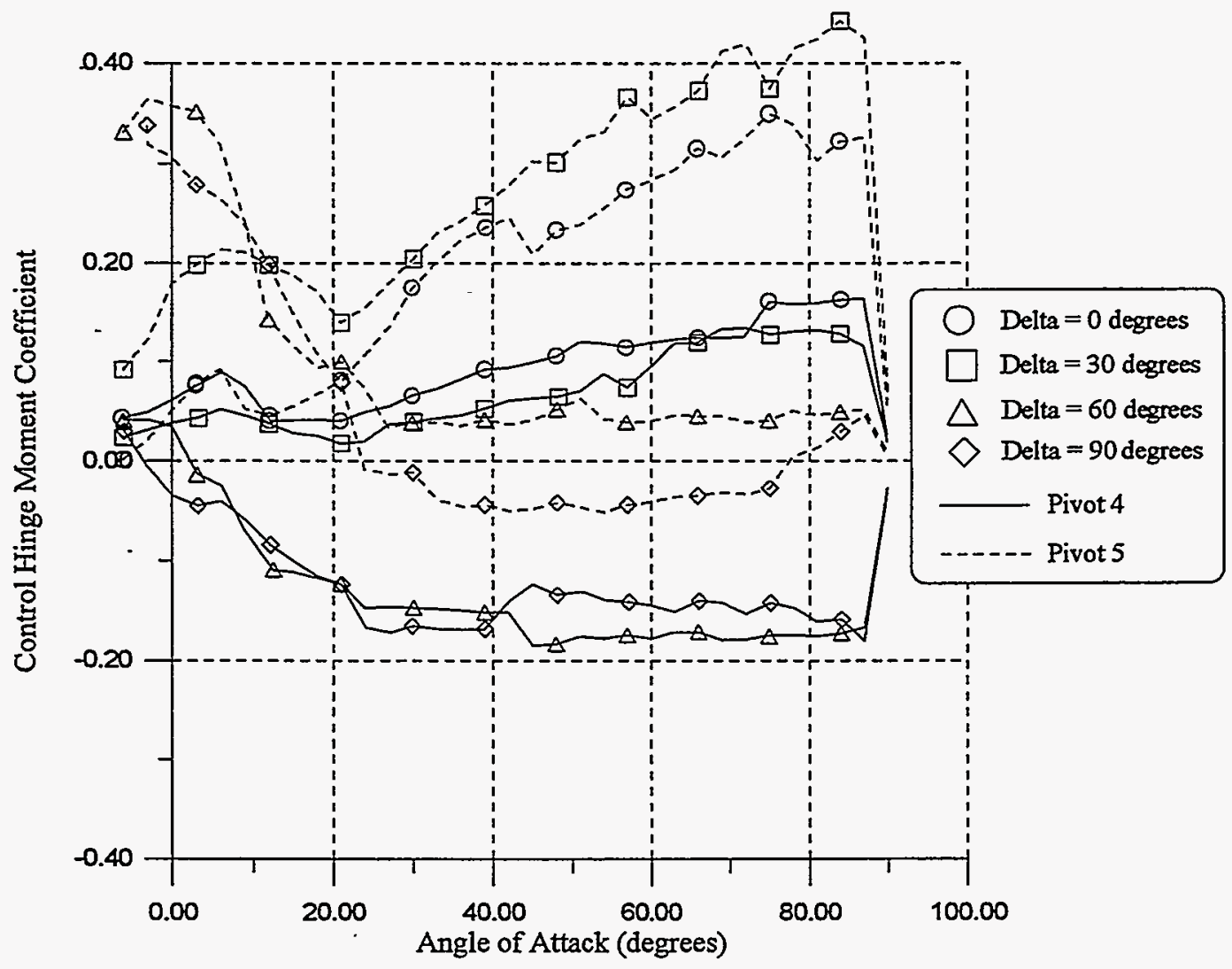

Figure 42. Impact of pivot point on $\mathbf{4 0} \%$ chord device hinge moment 


\section{Best Device Configuration}

On the basis of a concurrent data review performed during the wind tunnel test a "best" device configuration embodying a majority of desirable features was selected for closer evaluation and refinement. A $40 \%$ chord device, with a $1 \%$ lip length, 40 degree vent angle, and a pivot located at point 5 (aft-center) seemed to offer the best performance potential for wind turbine applications.

A good suction coefficient behavior over an angle of attack range from about 0 to 40 degrees favored use of a 1\% lip length and a vent angle of 40 degrees. As was noted previously, pivot 5 offers hinge moments that tend to automatically open the device. Such a situation suggests that passive device deployment and turbine overspeed protection is possible.

Selection of the $40 \%$ chord Spoiler-Flap configuration is perhaps more controversial and open to further review. The $50 \%$ chord device offers better turbine torque control for braking and power modulation applications, but the potential for performance losses during normal (i.e., $\delta=0$ ) turbine operations exists. Figures $43 \mathrm{a}$ and $43 \mathrm{~b}$ show the section drag coefficient for each device chord examined. As can be seen, the $50 \%$ chord device has greater drag than the $40 \%$ and $30 \%$ devices under typical turbine operating conditions. It is possible that the higher drag is due to a reduction in laminar flow or slight venting through the gap between the device and main airfoil elements. Interestingly, the drag performance improved and closely matched that of the $40 \%$ and $30 \%$ devices when the gap was sealed with tape. Selection of the $40 \%$ chord device as the "best" configuration for further evaluation seemed logical in light of these observations. Gap sealing or care to maintain a smooth surface geometry appear to be important if one uses a $50 \%$ chord device.

The basic suction, lift, and drag coefficient performance of the "best" configuration is shown in Figures $44 a, 44 b$, and 44c. In this case, the surface finish was smooth and thus represented a "clean" configuration. Figures 45-49 (a, b, and c) and 50-53 (a, b, and c) illustrate, respectively, the aerodynamic effect of surface roughness and of a Gurney flap. Device hinge moment coefficients, for the clean and Gurney flap cases, are shown in Figures $54(\mathrm{a}$ and $\mathrm{b}$ ) and 55. To more completely define the performance of this "best" configuration a larger number of device deflections was examined.

Number 60-grit roughness was applied to the leading-edges of both the main and device elements of the model using thin double-stick tape and an NREL supplied template. The Gurney flap, installed on the device lower-surface trailing-edge, was made from a small, 0.36-inch-high, L-shaped piece of aluminum.

\section{Aerodynamic Coefficients}

Figures $44 \mathrm{a}, 44 \mathrm{~b}$ and $44 \mathrm{c}$ illustrate that as the device deflection increases the suction and lift coefficient curves move in the negative direction. Indeed, the suction coefficient curves become negative and flatter over the entire angle of attack range. Interestingly, a jump in the progression of the curve motion occurs between a delta value of 30 and 45 degrees. A sudden change in the lift curve shape, as seen in Figure $44 b$, seems responsible for the observed sudden reduction in the suction coefficient. For angles of attack less than approximately 40 degrees, the drag coefficient appears to rise smoothly and without the abrupt jump observed in suction and lift coefficients as the device deflection increases. The jump behavior in the suction coefficient curves is possibly due to the fact that the device vents at a deflection of 40 degrees or that the device has moved past some critical value. 


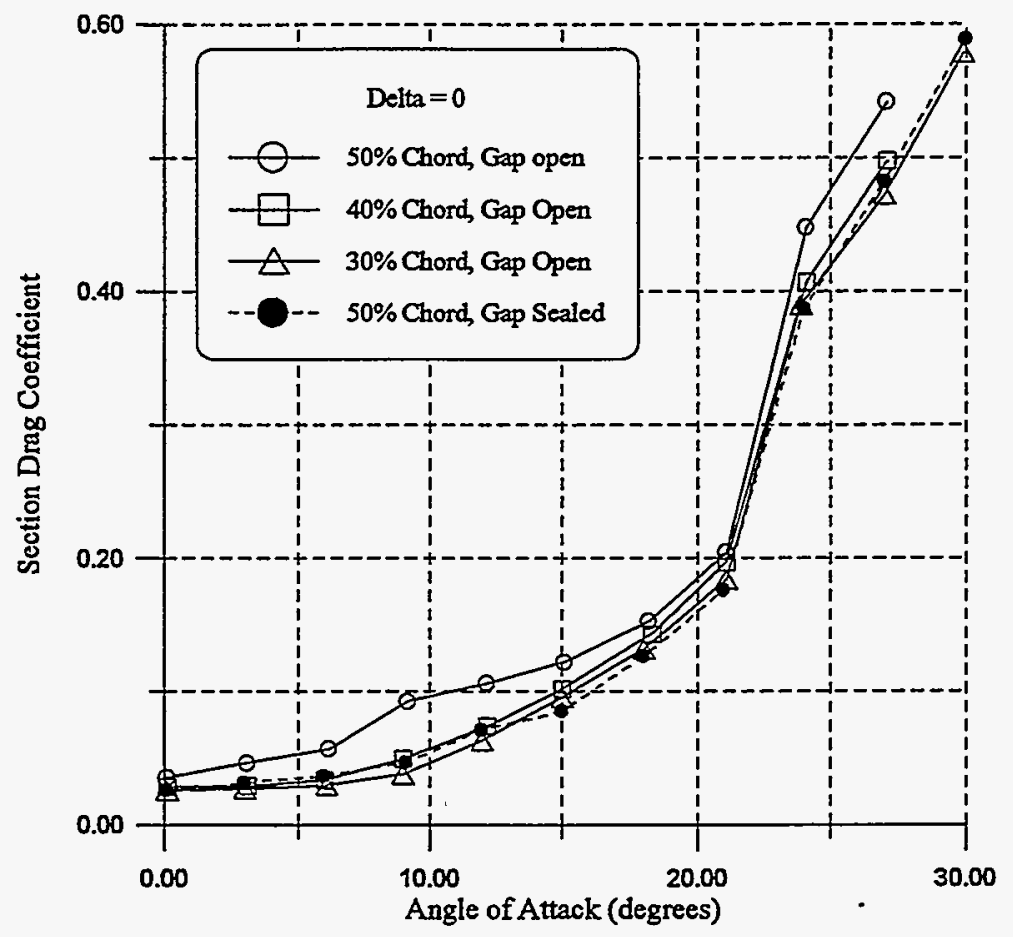

Figure 43a. Impact of device chord and gap sealing on drag, as a function of angle of attack

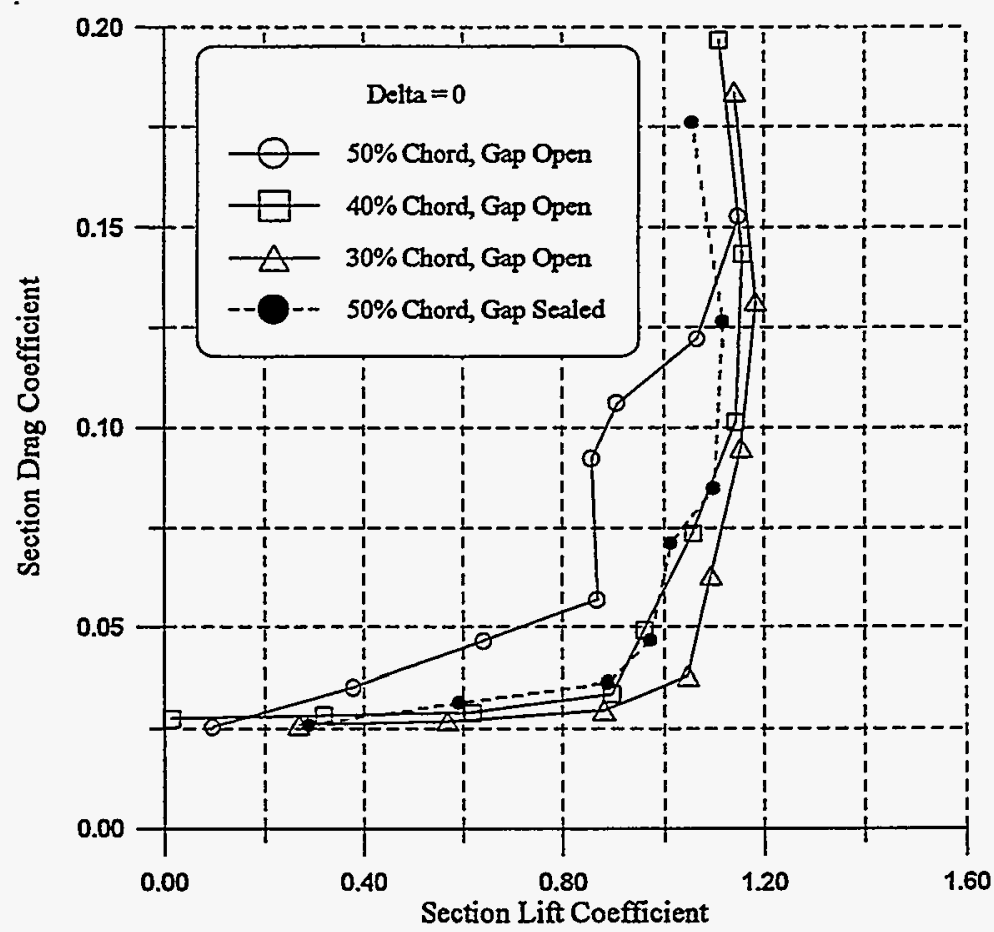

Figure 43b. Impact of device chord and gap sealing on drag, as a function of lift coefficient

Figure 43. Impact of device chord and gap sealing on drag 


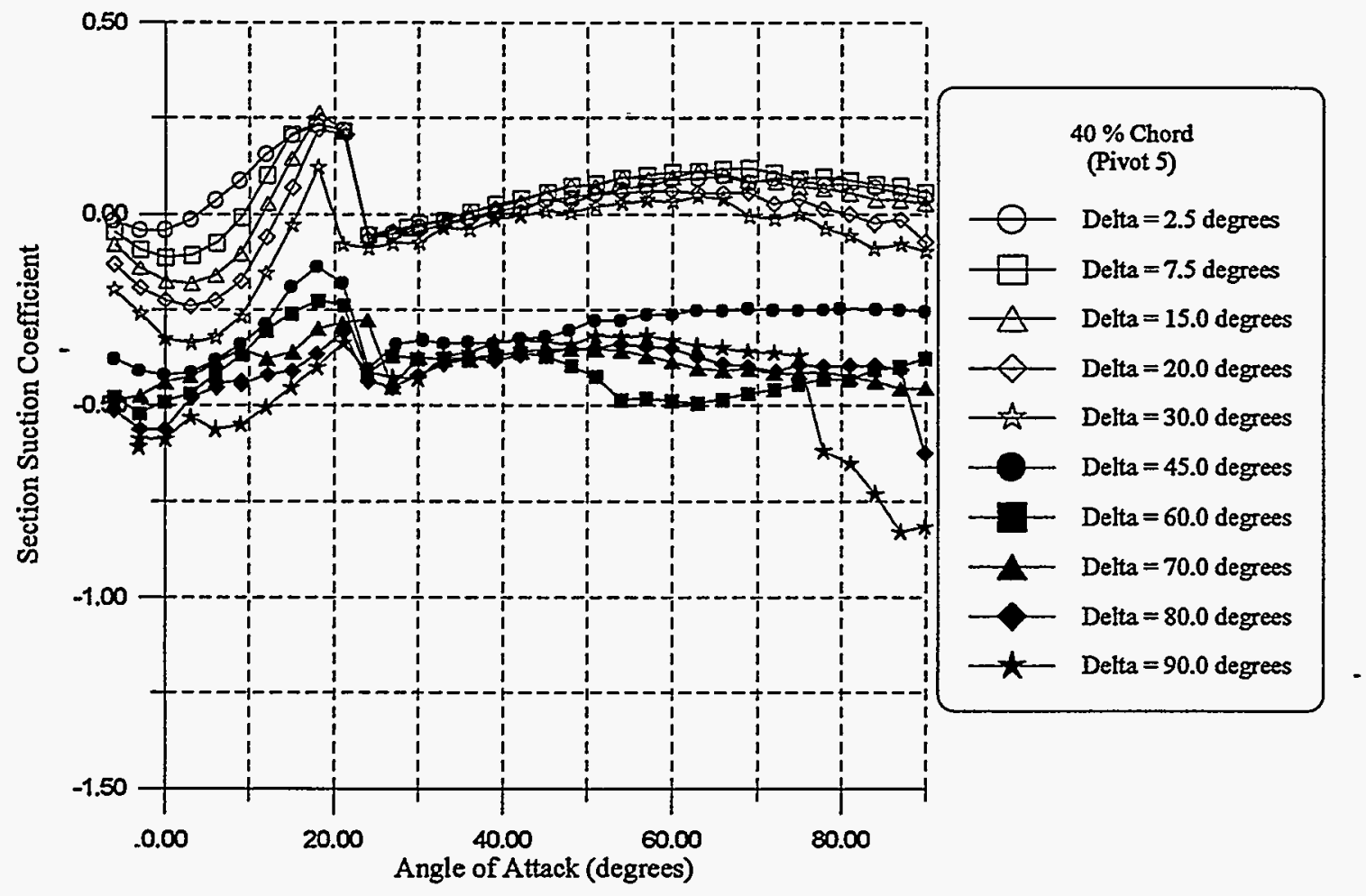

Figure 44a. Best device suction coefficient behavior

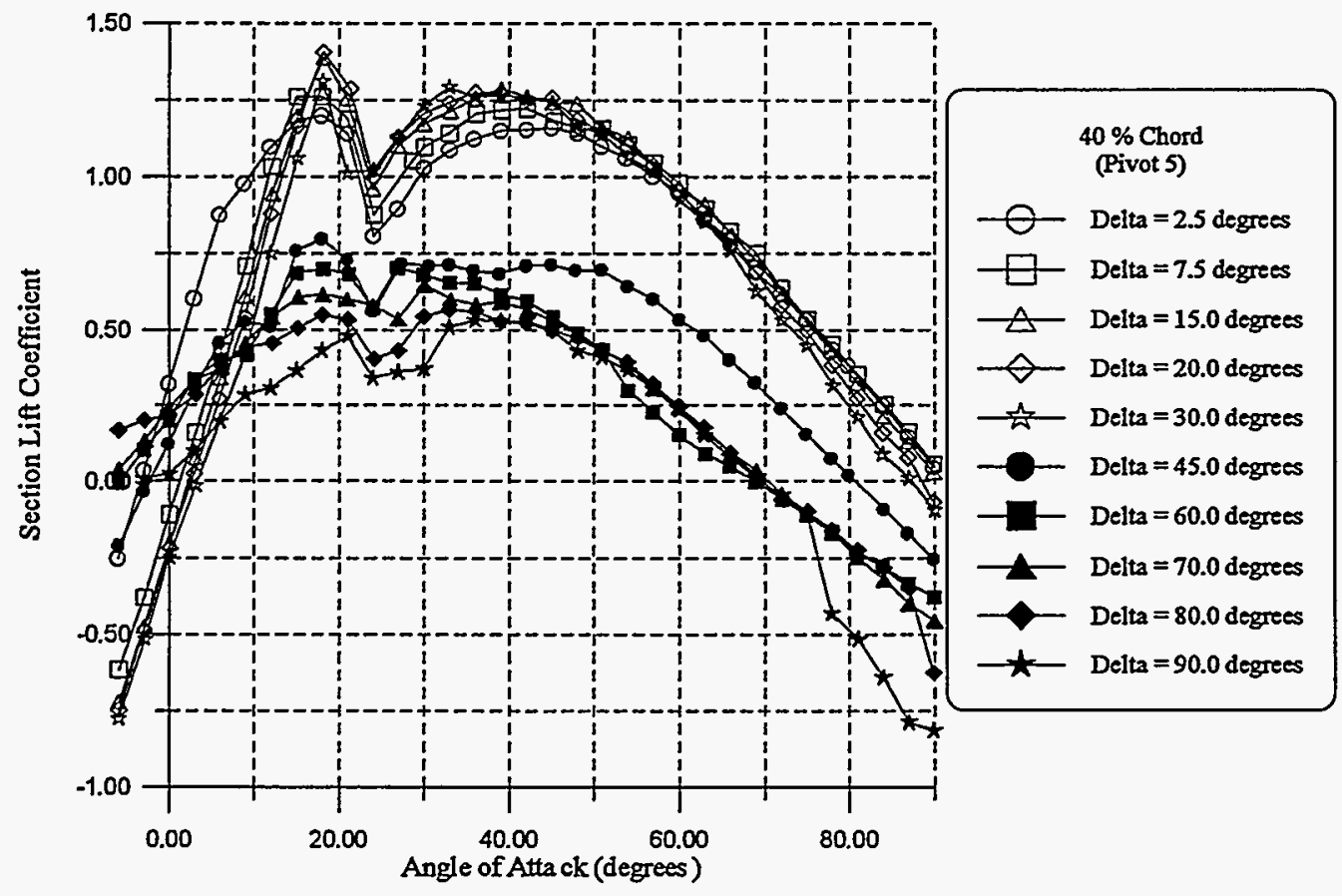

Figure 44b. Best device lift coefficient behavior 


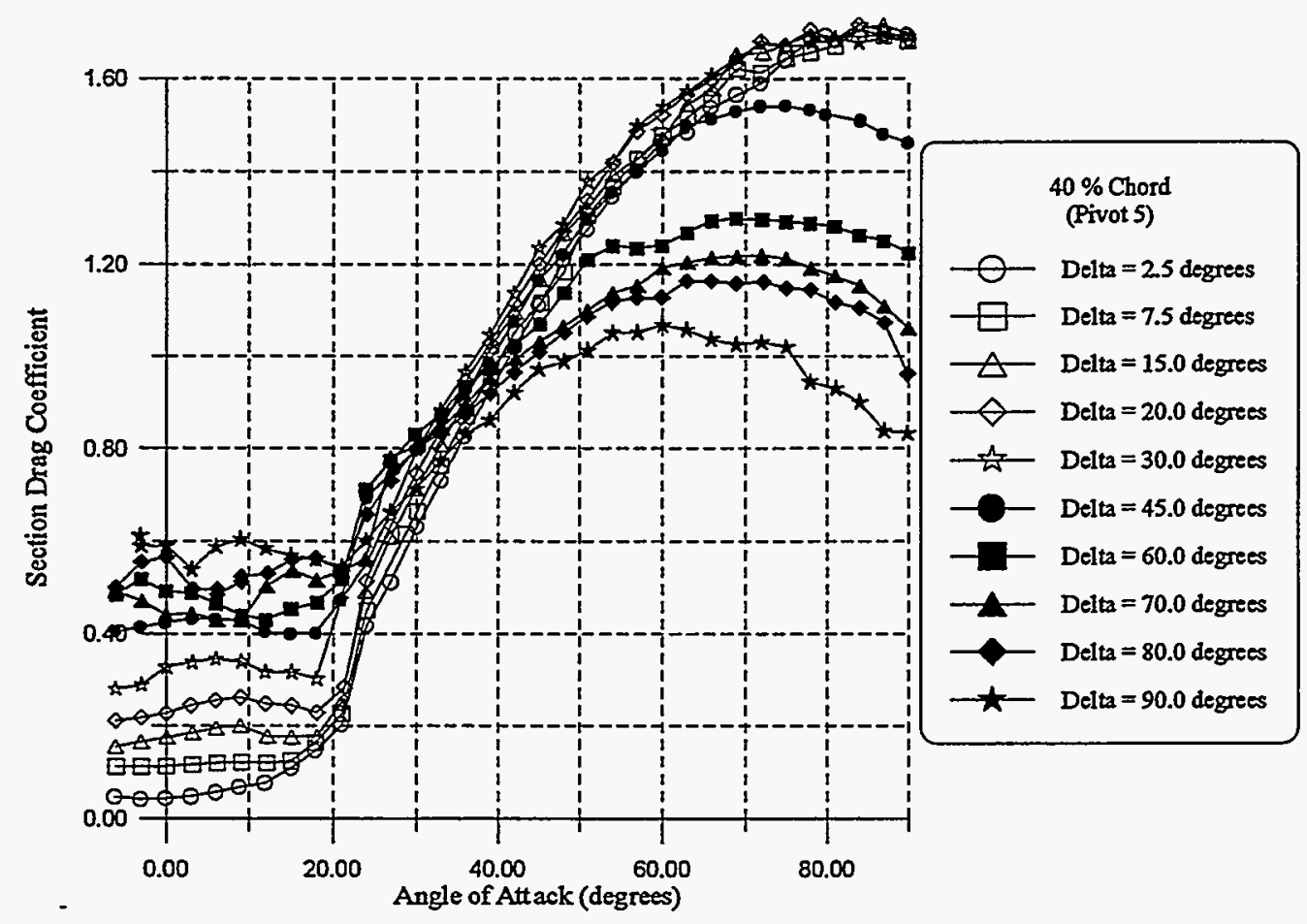

Figure 44c. Best device drag coefficient behavior

Figure 44. Best device aerodynamic coefficient behavior

\section{Roughness Effects}

The impact of roughness on the suction, lift, and drag coefficients is shown in Figures 45-49 ( $a, b$, and c). Differences are insignificant. The largest changes are observed for device deflections of less than 30 degrees and angles of attack between about 15 and 25 degrees. 


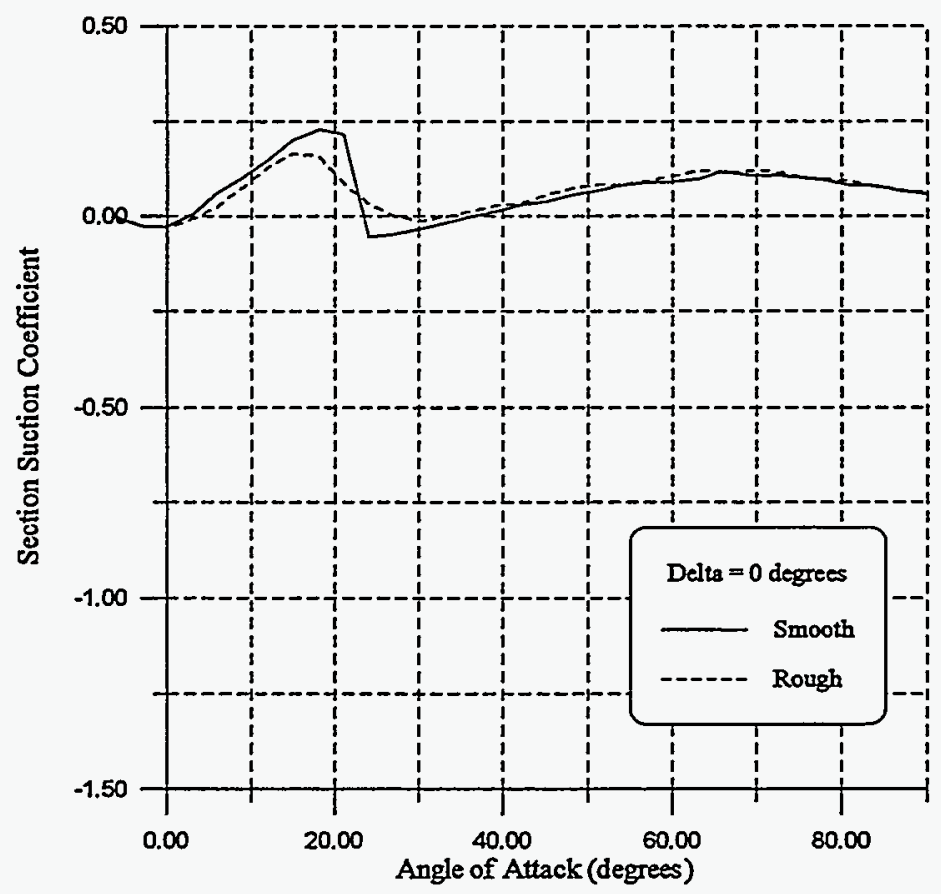

Figure 45a. Suction coefficient behavior

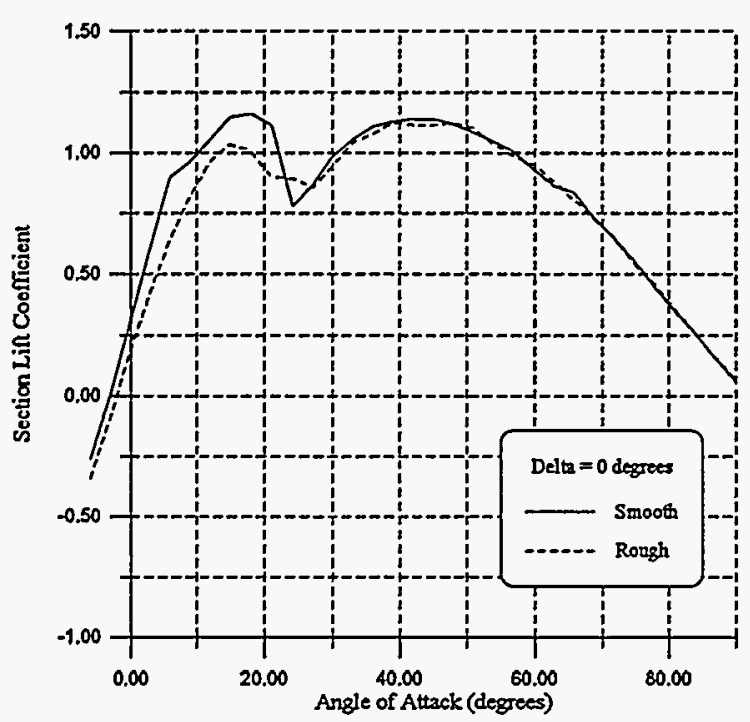

Figure 45b. Lift coefficient behavior

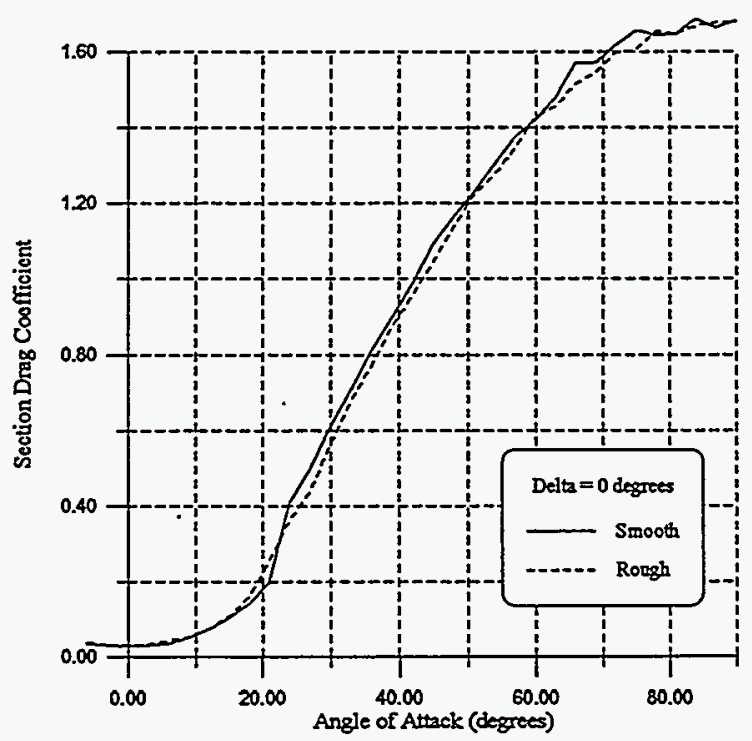

Figure 45c. Drag coefficient behavior

Figure 45. - Impact of roughness at a delta of 0 degrees 


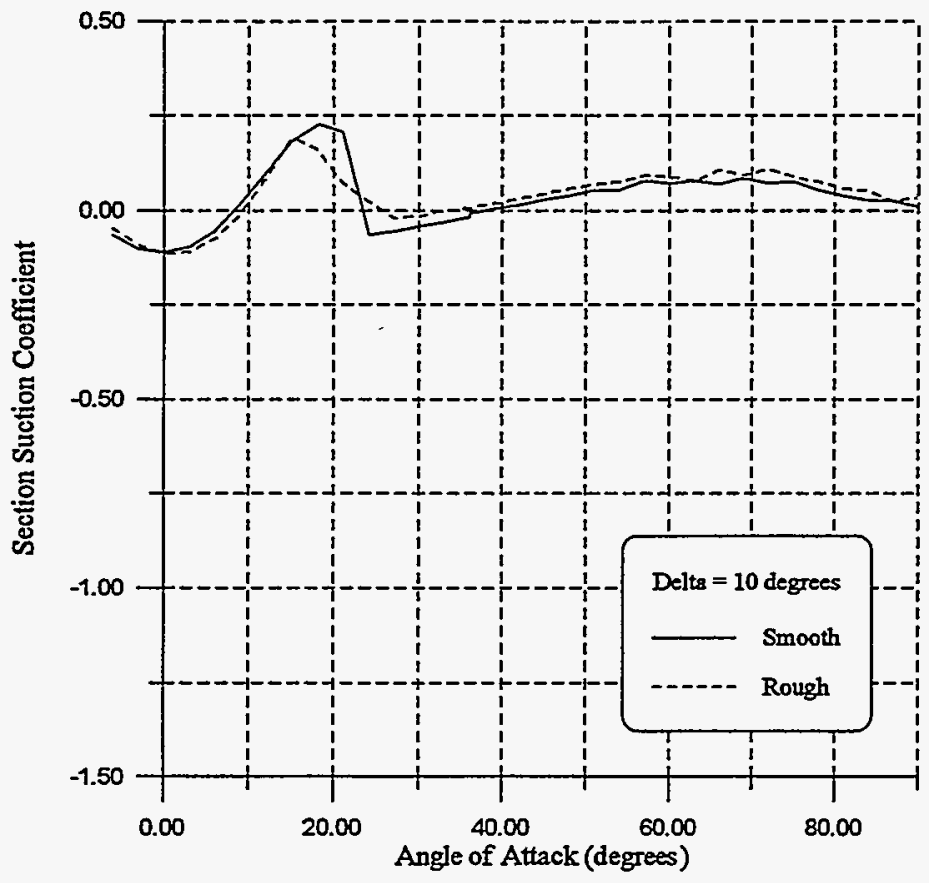

Figure 46a. Suction coefficient behavior

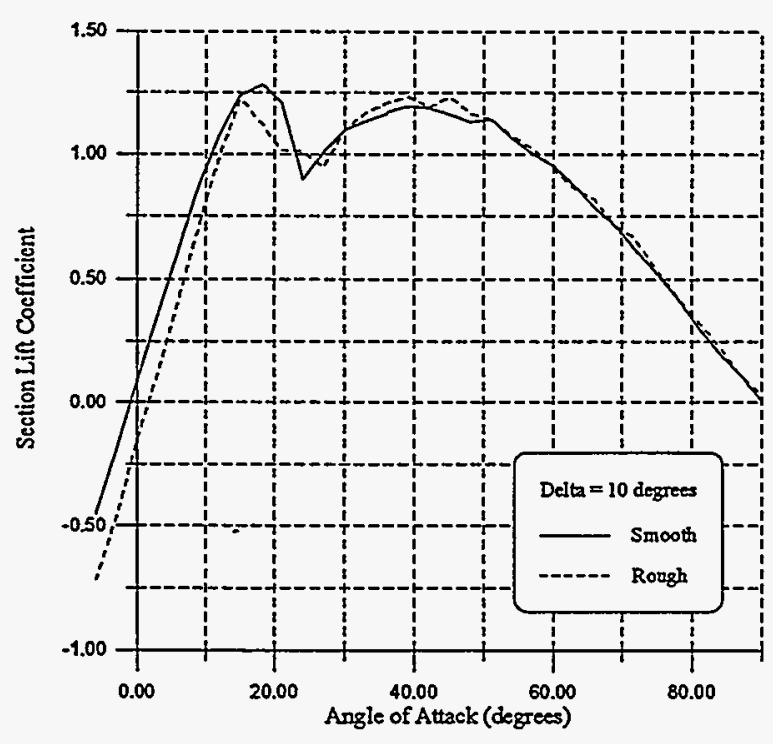

Figure 46b. Lift coefficient behavior

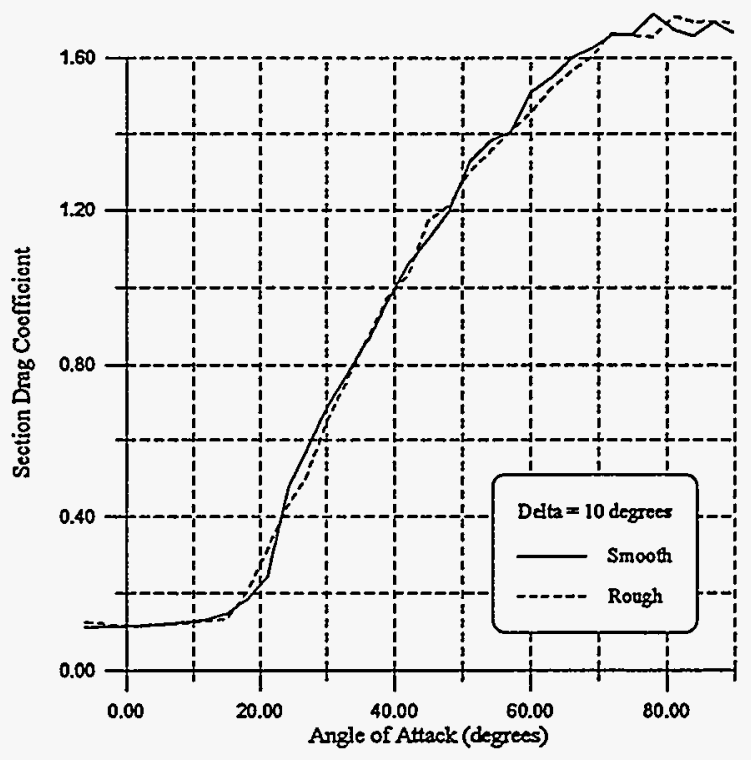

Figure 46c. Drag coefficient behavior

Figure 46. Impact of roughness at a delta of 10 degrees 


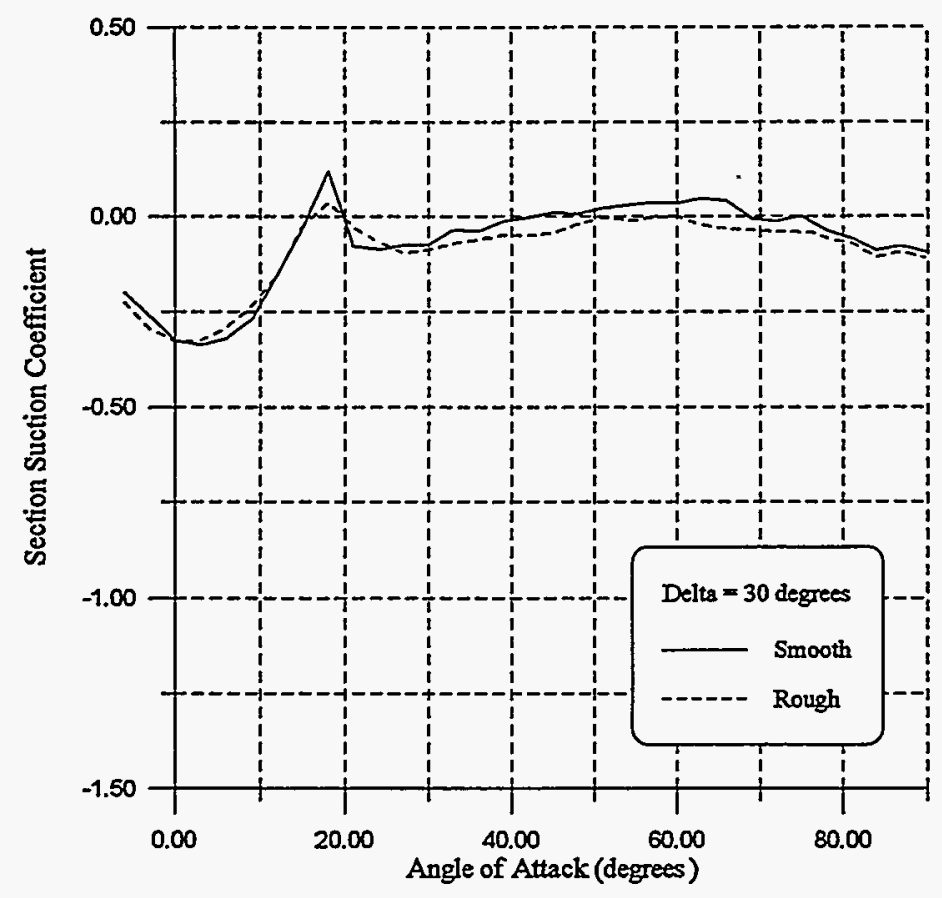

Figure 47a. Suction coefficient behavior

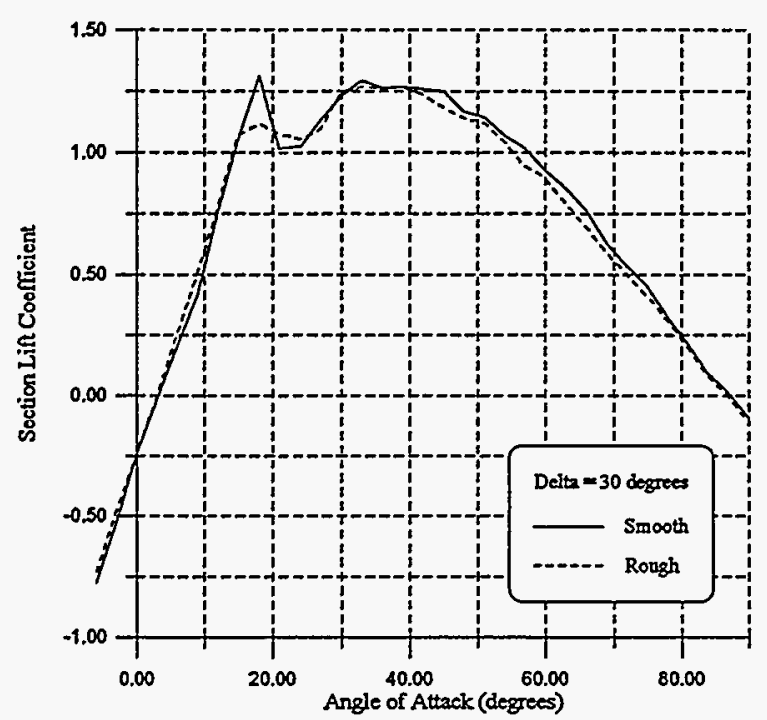

Figure $47 \mathrm{~b}$. Lift coefficient behavior

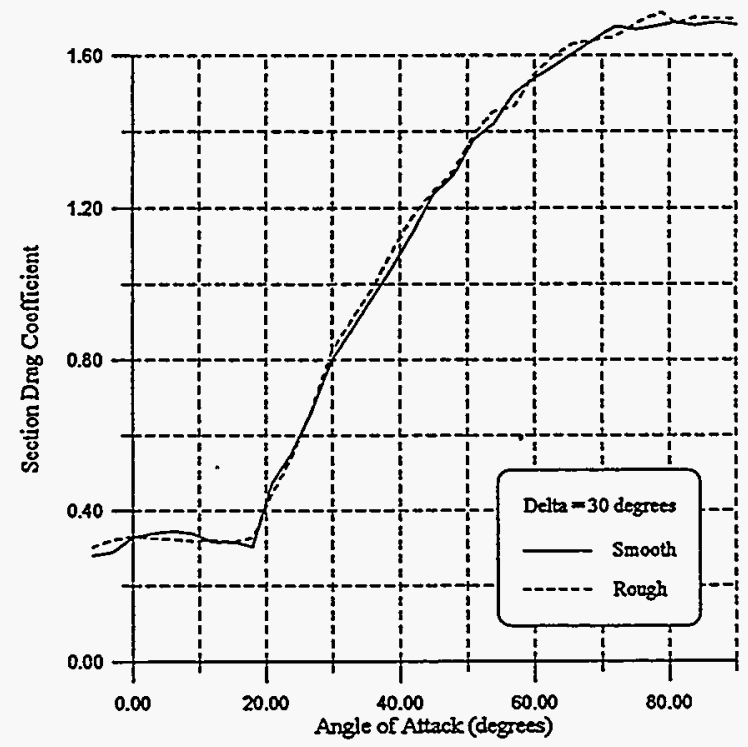

Figure 47c. Drag coefficient behavior

Figure 47. Impact of roughness at a delta of $\mathbf{3 0}$ degrees 


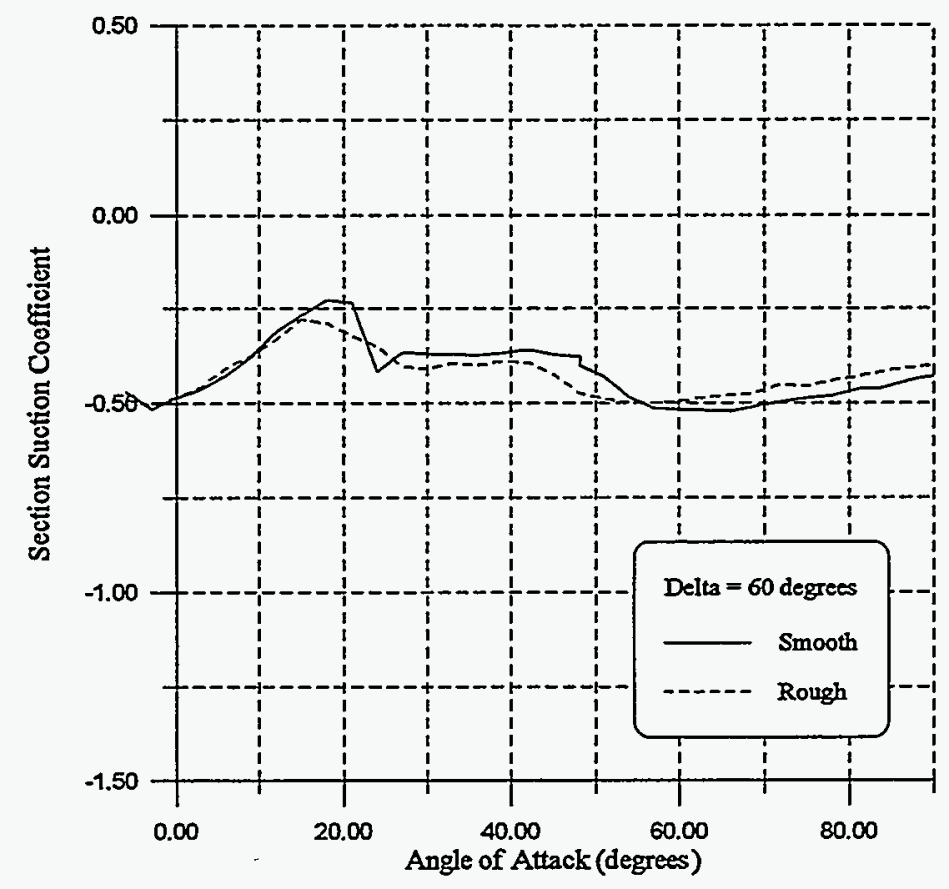

Figure 48a. Suction coefficient behavior

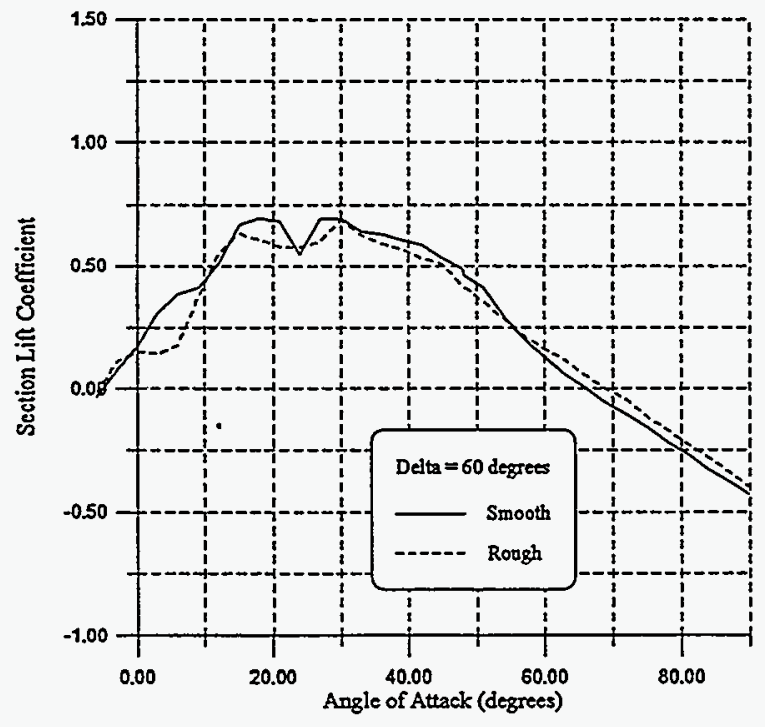

Figure 48b. Lift coefficient behavior

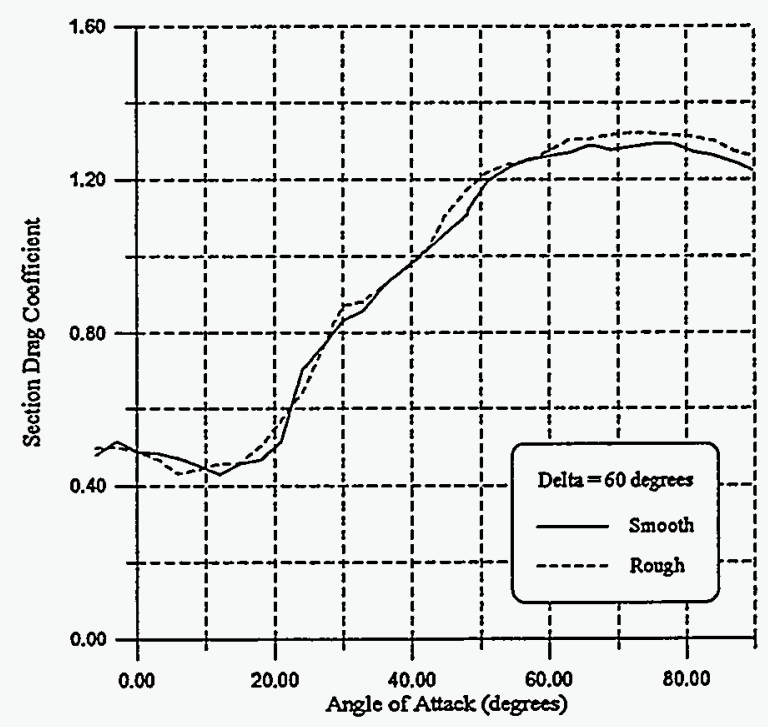

Figure 48c. Drag coefficient behavior

Figure 48. Impact of roughness at a delta of 60 degrees 


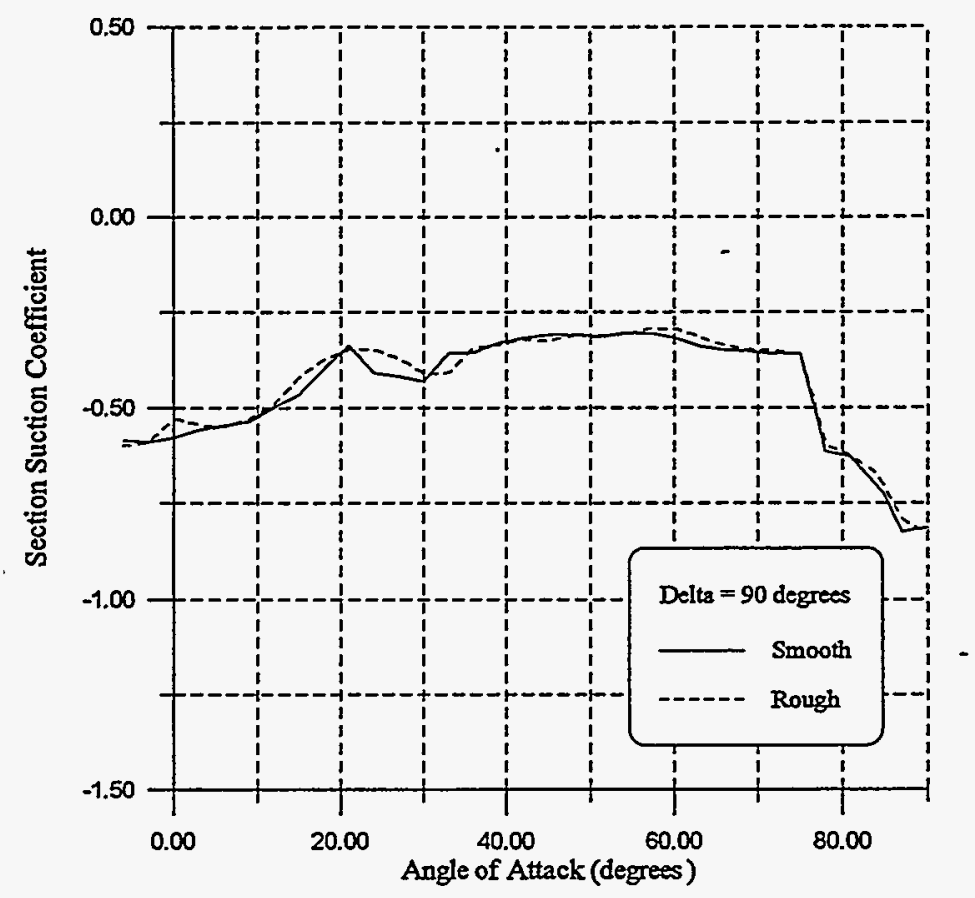

Figure 49a. Suction coefficient behavior

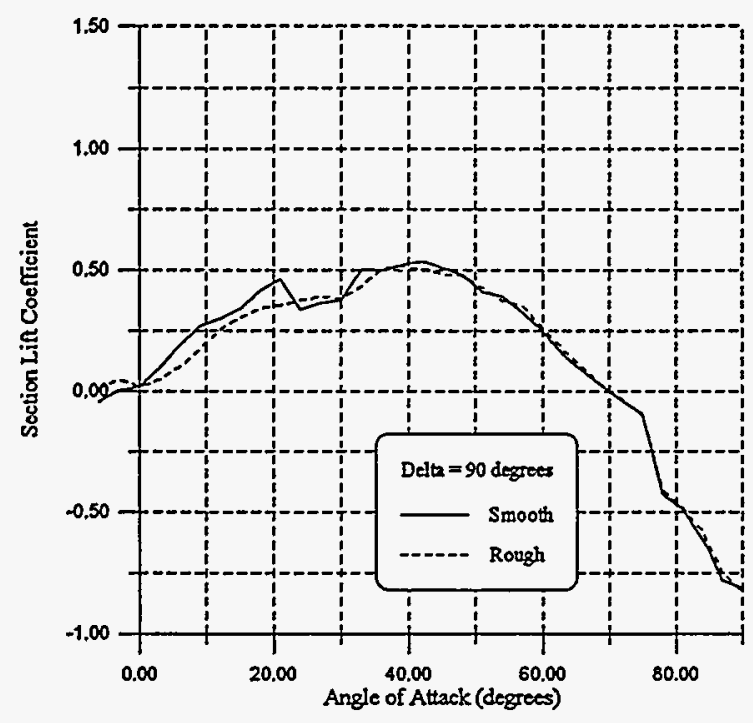

Figure $49 \mathrm{~b}$. Lift coefficient behavior

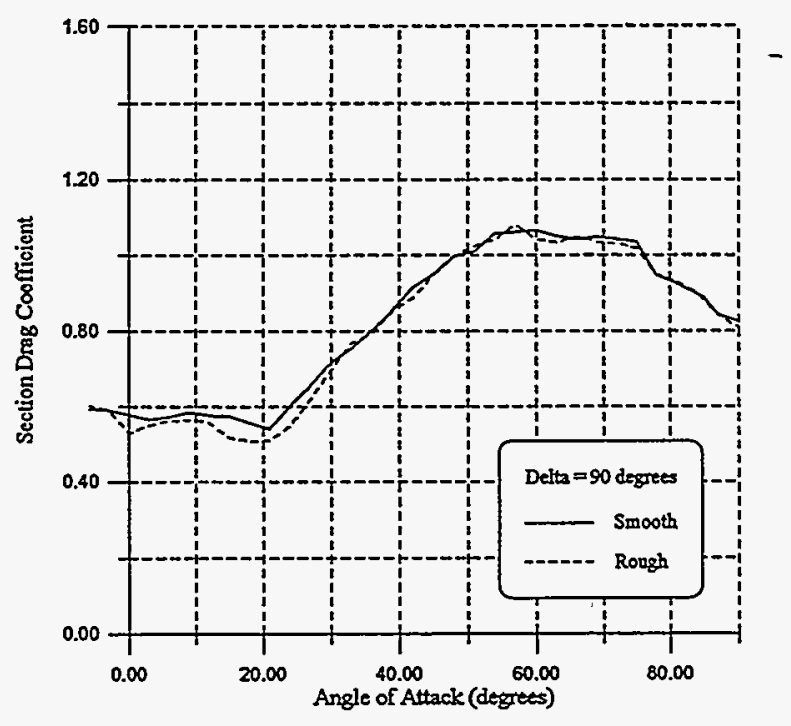

Figure 49c. Drag coefficient behavior

Figure 49. Impact of roughness at a delta of 90 degrees 


\section{Gurney Flap}

As Figures $50-51(\mathrm{a}, \mathrm{b}$, and $\mathrm{c})$ show, the Gurney flap has only a minor impact on the suction coefficient character for device deflections of 30 degrees or less. Interestingly, a close examination of the data indicates that both the lift and drag coefficients were increased by the Gurney flap and, as a result, the net effect on the suction coefficient is small. An increase in only the drag coefficient would have been preferred.

For device deflections greater than 30 degrees the effect of the Gurney flap on the suction, lift, and drag coefficient behavior becomes negligible. This situation is illustrated in Figures 52 and $53(\mathrm{a}, \mathrm{b}$, and c).

It is possible that Gurney flap height adjustments might offer performance improvements during both normal turbine operating conditions and turbine overspeed or power modulation situations. Unfortunately, time limitations prevented further examination of this possibility. 


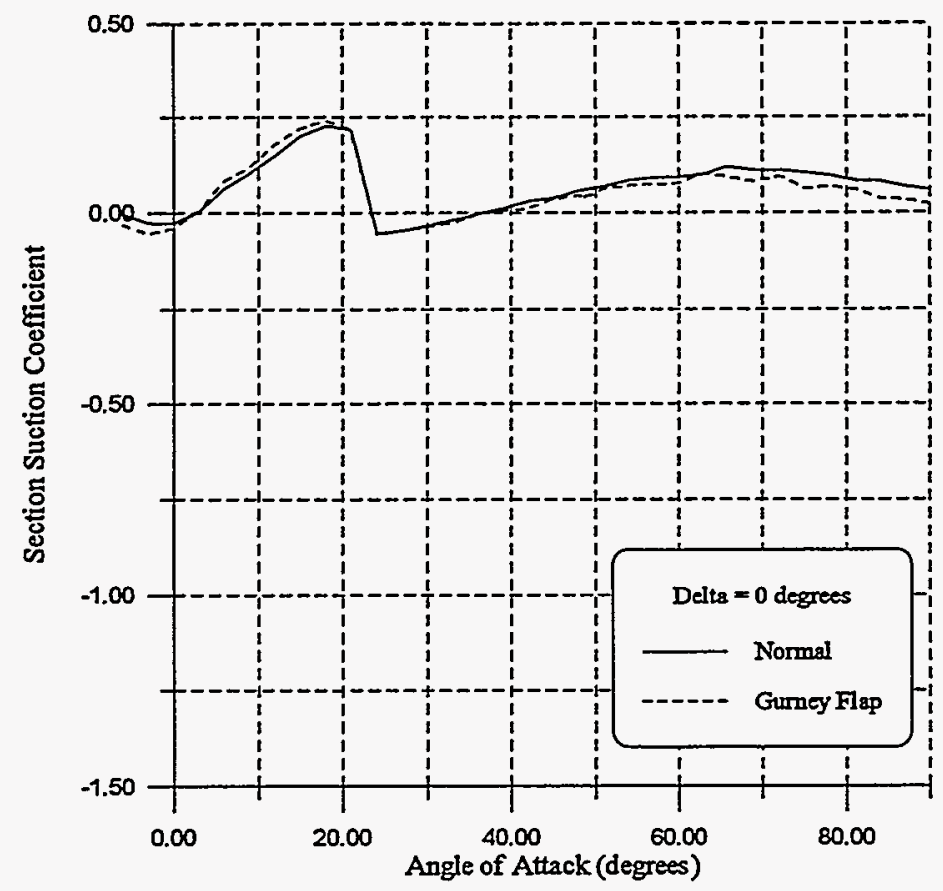

Figure 50a. Suction coefficient behavior

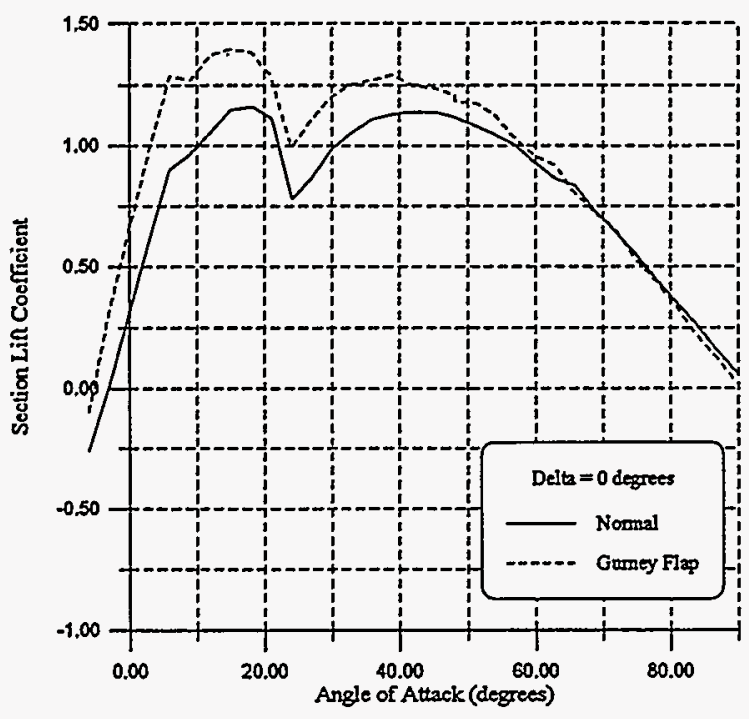

Figure 50b. Lift coefficient behavior

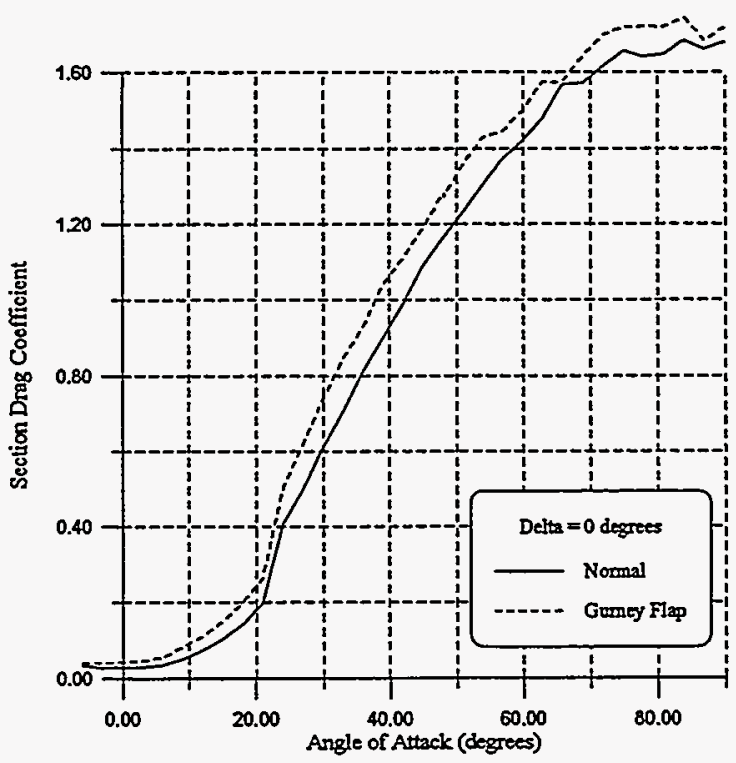

Figure 50c. Drag coefficient behavior

Figure 50. Impact of a Gurney flap at a delta of 0 degrees 


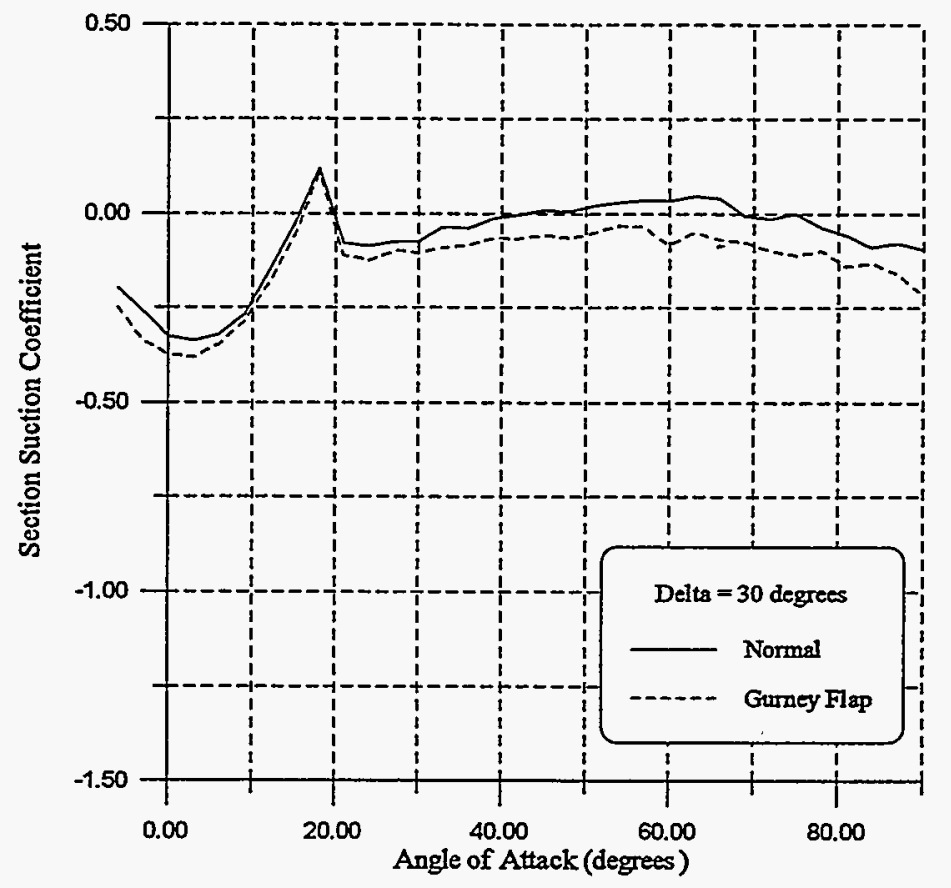

Figure 51a. Suction coefficient behavior

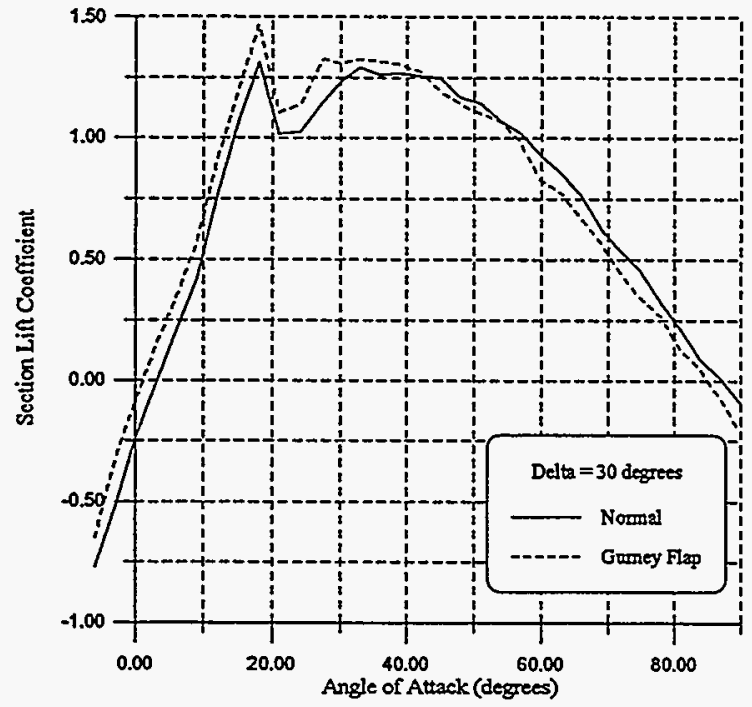

Figure 51b. Lift coefficient behavior

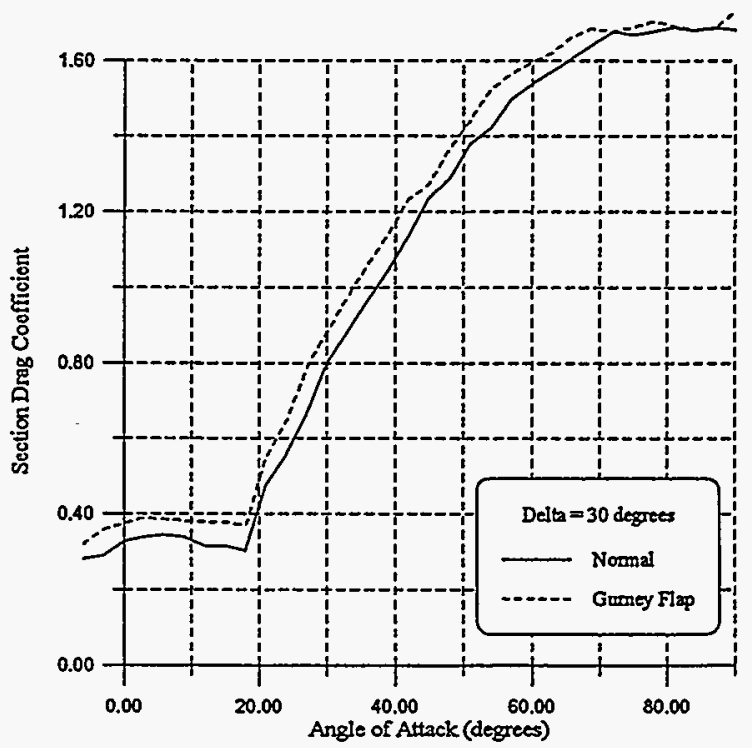

Figure 51c. Drag coefficient behavior

Figure 51. Impact of a Gurney flap at a delta of $\mathbf{3 0}$ degrees 


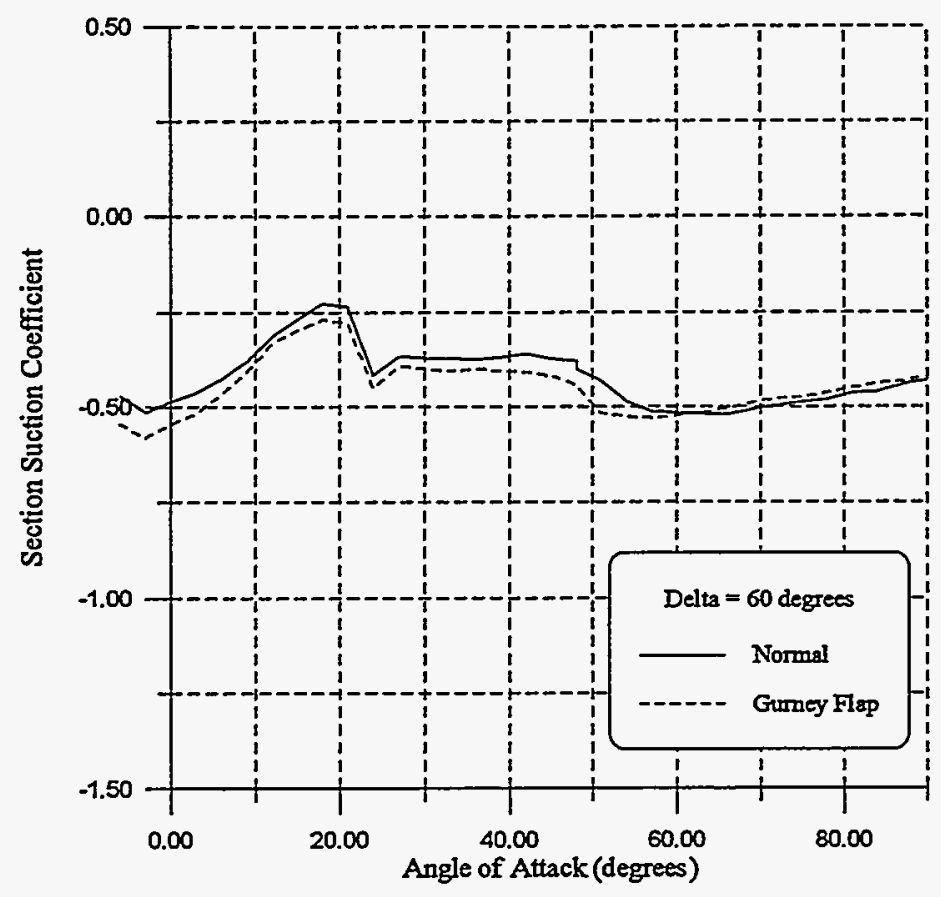

Figure 52a. Suction coefficient behavior

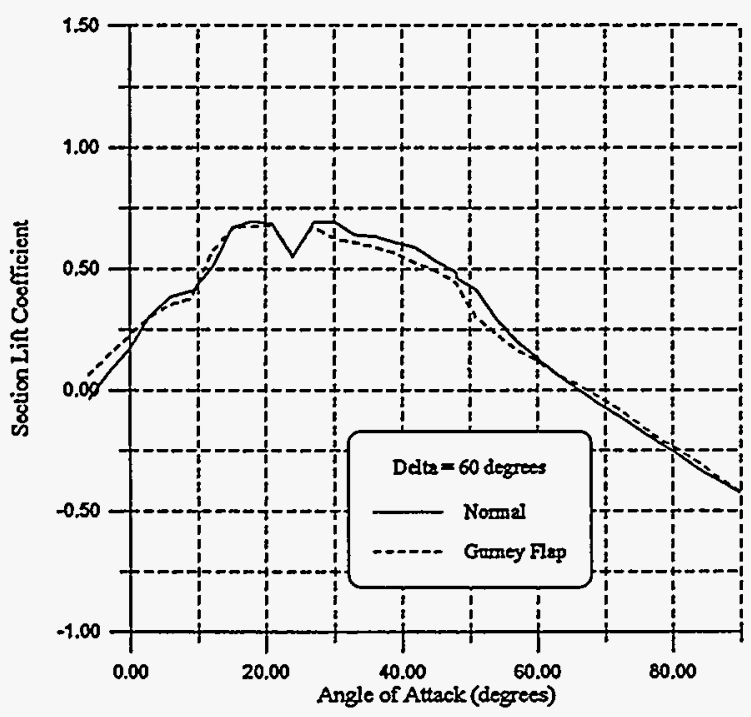

Figure 52b. Lift coefficient behavior

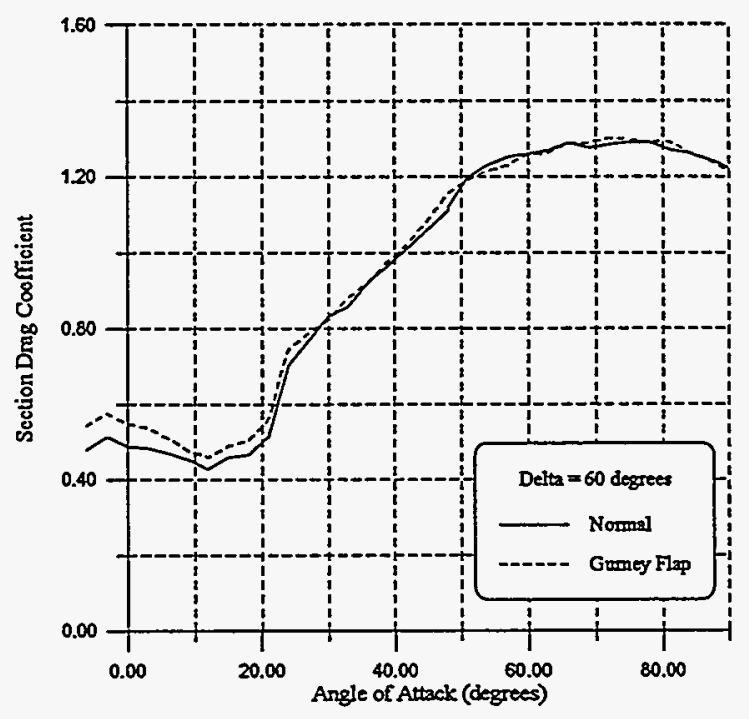

Figure 52c. Drag coefficient behavior

Figure 52. Impact of a Gurney flap at a delta of 60 degrees 


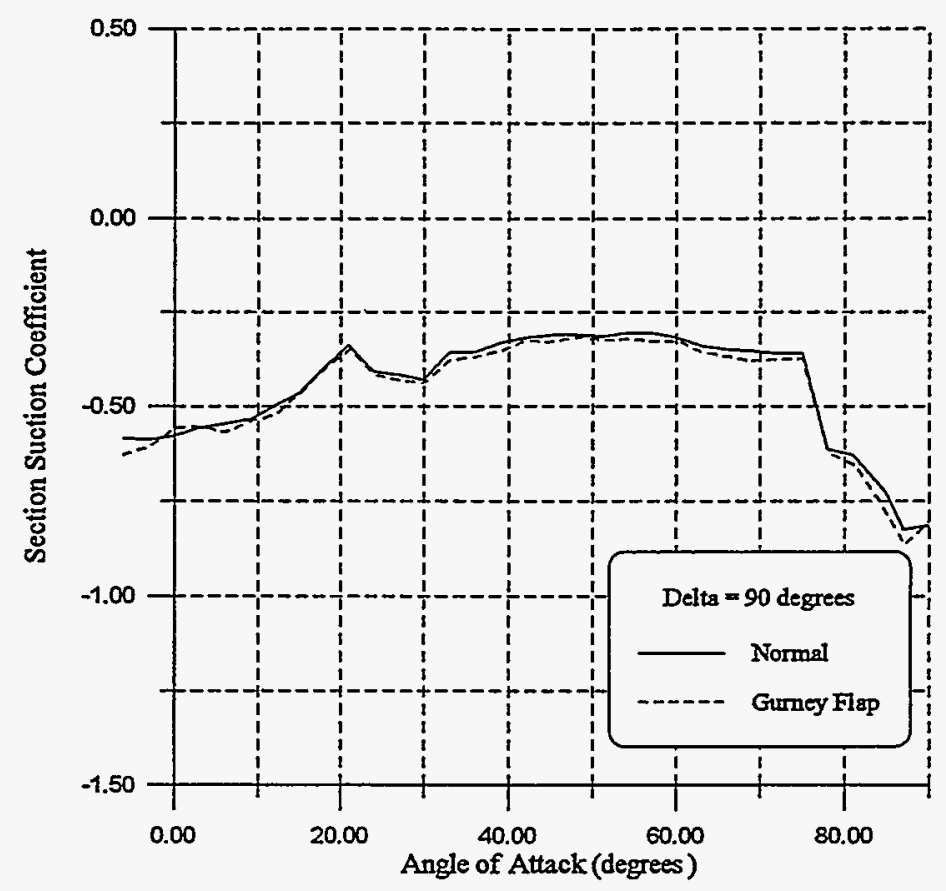

Figure 53a. Suction coefficient behavior

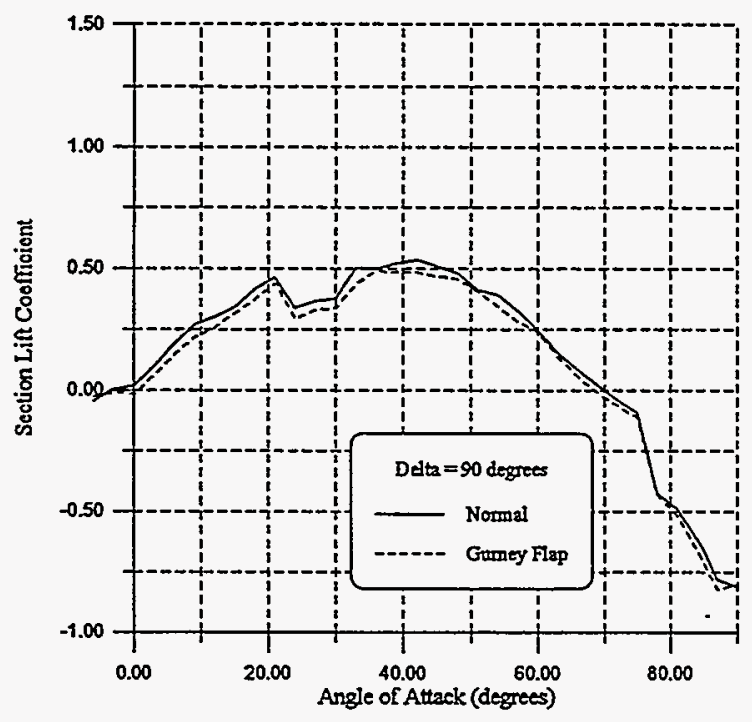

Figure 53b. Lift coefficient behavior

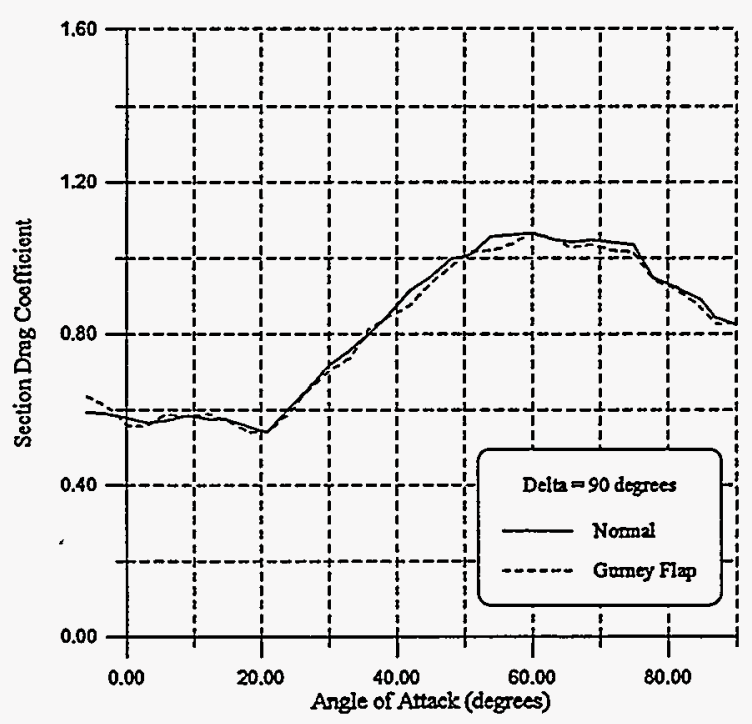

Figure 53c. Drag coefficient behavior

Figure 53. Impact of a Gurney flap at a delta of 90 degrees 


\section{Hinge Moment}

The hinge moment behavior of the best device is shown in Figures $54 \mathrm{a}$ and $54 \mathrm{~b}$. Figure 55 identifies the impact of a Gurney flap. Note that the Figures $54 \mathrm{~b}$ and 55 use different ordinate ranges, in order to provide a close-up views of the moment behavior.

As Figures 54a and 54b illustrate, the hinge moment coefficients are positive for all device deflections except 90 degrees. The Gurney flap has the potentially undesirable effect of making the hinge moments more negative. Recall that a positive hinge moment coefficient indicates that the device would open automatically if not restrained.

The hinge moment coefficients for this "best" device configuration are much improved over those originally obtained in the Phase 1 work. However, whether the magnitudes are the best possible is debatable. In light of the need to actuate the device, on a functioning turbine, it might be desirable to reduce the hinge moment coefficient magnitude even further from the levels shown in Figures $54 \mathrm{a}$ and $54 \mathrm{~b}$.

A simple example, for a typical $250 \mathrm{~kW}$ class turbine (with an 80.0 foot diameter rotor, 1.5 foot chord blades, $40 \%$ chord and $20 \%$ span devices, and an operating speed of $60 \mathrm{rpm}$ ) illustrates the importance of minimizing the magnitude of device hinge moment coefficients. Given a hinge moment coefficient value of 0.1 , an actuator capable of approximately 200 in- $1 \mathrm{~b}$ of torque is required to drive the Spoiler-Flap device. Figures 54a and 54b show that the current "best" device has coefficients of this magnitude and up to four times greater. A notably lower moment coefficient seems desirable in order to minimize device-actuator size, weight, and cost. It is conceivable that a different pivot location would yield more favorable moment values. Unfortunately, time and model limitations prevented testing at other device pivot points.

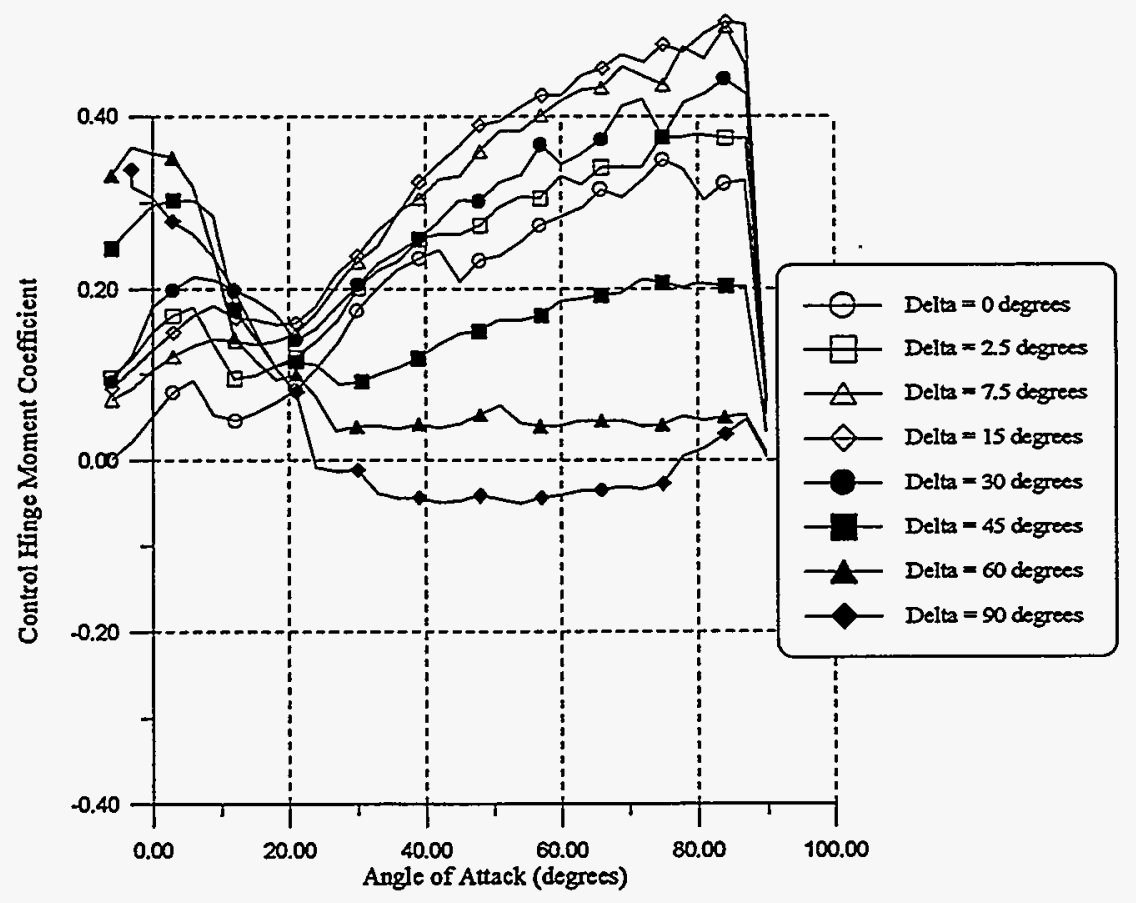

Figure 54a. Hinge moment behavior 


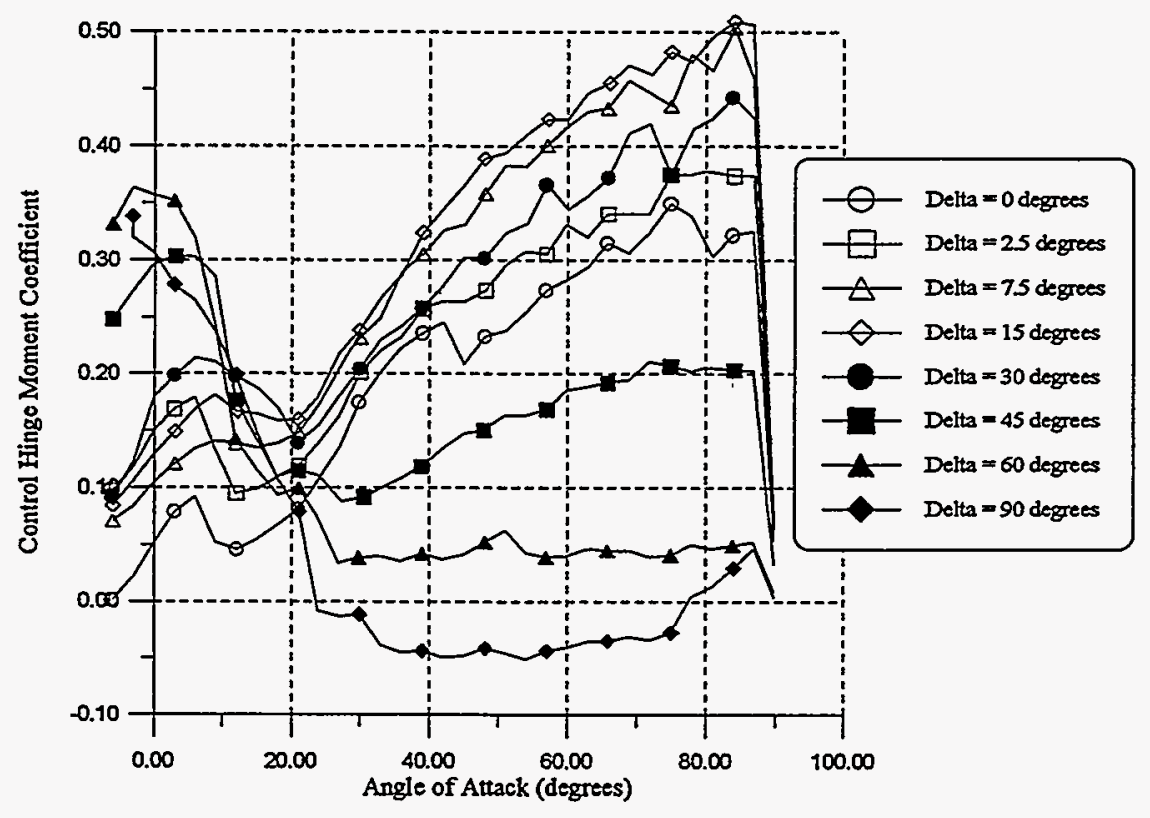

Figure 54b. Hinge moment behavior (close-up)

Figure 54. Hinge moment behavior as a function of angle of attack and delta

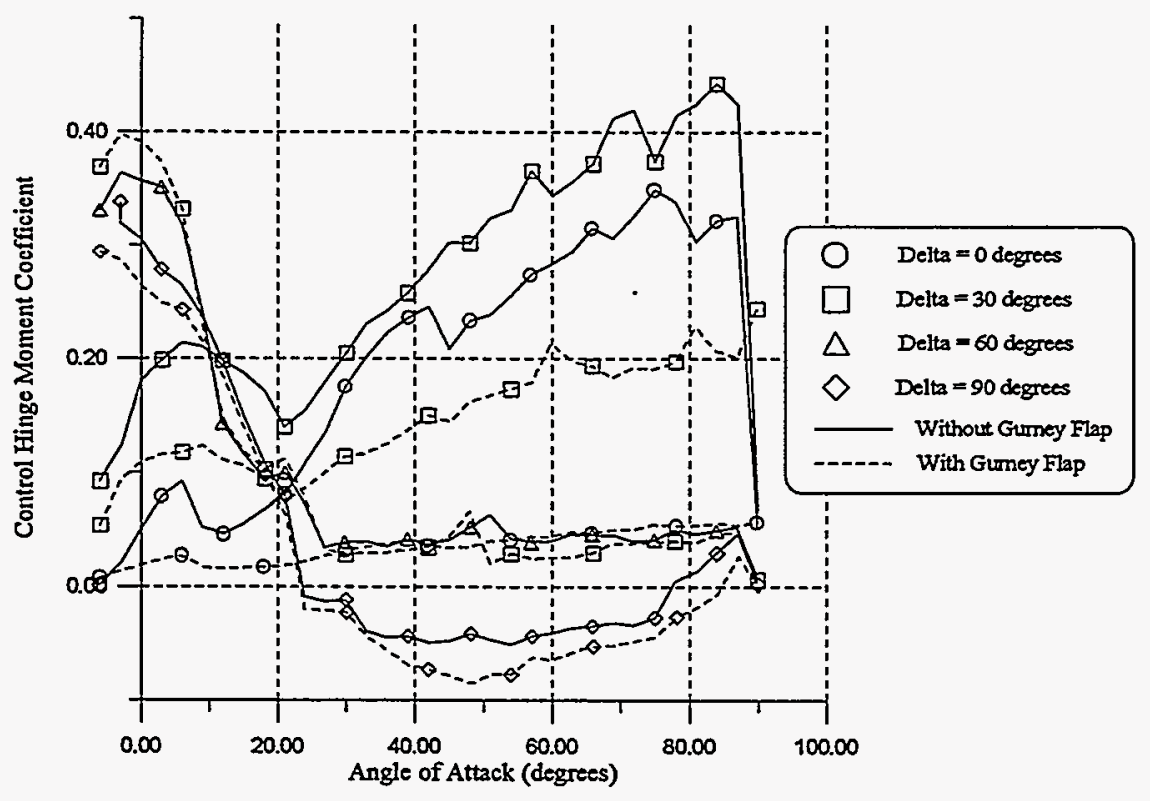

Figure 55. Impact of Gurney flap on hinge moment behavior (close-up) 


\section{Conclusions}

An experimental investigation was performed in an attempt to improve the Spoiler-Flap aerodynamic device, for power modulation and overspeed protection applications on wind turbines. The impact of device lip length, vent angle, pivot location, and chord were examined. A significant amount of experimental data was gathered including suction, lift, drag, and hinge moment coefficients as a function of device deflection and angle of attack. In addition, the impact of surface roughness and a Gurney flap were evaluated. On the basis of the results, the following conclusions are offered.

\section{Lip Length Effects}

1) Lip length increments for the $50 \%$ chord device have only a secondary impact on the aerodynamic performance. Changes are most pronounced for small (i.e., less than 10 degrees) device deflections and angles of attack above 15 degrees.

2) An increased lip length on the shorter (i.e., $40 \%$ and $30 \%$ ) chord devices has only a minor affect on the aerodynamic performance. Changes are small and limited primarily to power producing angles of attack of less than 20 degrees.

3) For device-deflections of less than 60 degrees and low angles of attack (i.e., less that 10 degrees) a longer lip makes the hinge moments more positive. However, use of a longer lip tends to make the device hinge moments more negative in magnitude (i.e., the device is harder to keep open) at angles of attack above about 10 degrees.

\section{Vent Angle Effects}

4) The impact of vent angle on the aerodynamic performance, for the $50 \%$ chord device, is most noticeable for device deflections greater than 60 degrees. Delayed venting (i.e., a larger vent angle) appears to improve the suction coefficient performance, especially for angles of attack between 15 and 35 degrees (i.e., for turbine aerodynamic braking applications).

5) Vent angle effects on the aerodynamic performance are relatively minor and primarily limited to angles of attack greater than 30 degrees, for the $40 \%$ chord device.

6) Delayed venting is preferred if automatic opening of the device, as for passive turbine overspeed protection, is desired. Early venting makes the device hinge moments more negative (i.e., the device is harder to keep open).

\section{Chord Effects}

7) A longer chord device appears to offer better all-around suction coefficient performance for both turbine braking and power modulation applications. A progressive reduction in aerodynamic capability is observed as the chord is reduced from $50 \%$. The chord effects are most pronounced at angles of attack below about 35 degrees. 
8) Detailed analysis shows that the $50 \%$ chord Spoiler-Flap offers the greatest normalized device effectiveness at angles of attack less than about 30 degrees. Interestingly, the shorter $30 \%$ chord device is most effective at angles of attack above about 35 degrees. The normalized effectiveness of the $40 \%$ chord device is less than the $50 \%$ device, but notably better than that of the $30 \%$.

9) The magnitude of the hinge moment coefficient for the $40 \%$ chord device is typically more positive than that of the $50 \%$ chord device (i.e., the device is usually easier to keep open).

\section{Pivot Point Effects}

10) Device pivot point location, as examined in this investigation, has only a minor impact on the aerodynamic performance of the $40 \%$ and $50 \%$ chord devices.

11) Aft movement of the pivot point has a dramatic and favorable impact on the device hinge moment behavior (i.e., the device becomes easier to open). This specific result is significant and suggests that an opportunity exists to tailor device hinge moments without sacrificing aerodynamic performance.

\section{"Best" Device}

12) On the basis of a concurrent data review performed during the wind tunnel test, a "best" device configuration embodying a majority of desirable features was selected for closer evaluation and refinement. A $40 \%$ chord device, with a $1 \%$ lip length, 40 degree vent angle, and a pivot located at point 5 (aft-center) was assumed to offer the best performance potential for wind turbine power modulation and overspeed (i.e., braking) applications.

13) Use of a larger chord device (i.e., 50\%) requires careful gap sealing in order to minimize section drag coefficient increases at low angles of attack typical of turbine power-producing conditions.

14) The "best" device configuration hinge moment values are improved over earlier Spoiler-Flap configurations. However, the magnitude of the measured moment coefficients still seem excessive, especially for wind turbine applications. It is possible that another pivot point, besides pivot 5 , might yield smaller and more reasonable hinge moments.

15) Surface roughness reduced the suction coefficient behavior notably for device deflections and angles of attack of less than about 25 degrees. These conditions are typical of power producing and power modulation. The impact of surface roughness was found to be minimal for braking situations, where large device deflections and high angles of attack exist.

16) The observed performance effect of the Gurney flap was small. However, only one Gurney flap size was examined and it is conceivable that another configuration might perform better. 


\section{References}

Miller, L.S. (1995). Experimental Investigation of Aerodynamic Devices for Wind Turbine Rotational Speed Control: Phase 1. NREL/TP-441-6913. Golden, CO: National Renewable Energy Laboratory.

Johnson, B.L. (1993). Facility Description of the Walter H. Beech 7X10 Foot Low-Speed Wind Tunnel. Wichita, KS: Aerodynamic Laboratory, Wichita State University, 1845 N. Fairmount, Wichita, KS, 67260-0044 (316-689-3404).

Rae, W.H., Pope A. (1984). Low-Speed Wind Tunnel Testing, 2nd Edition. New York: John Wiley \& Sons. 


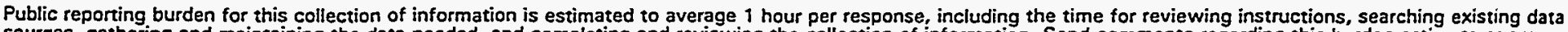

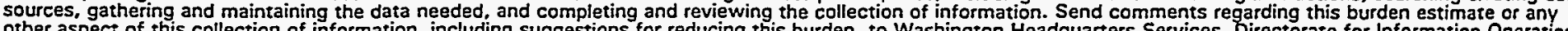

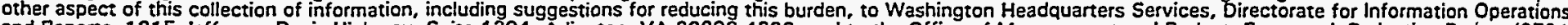

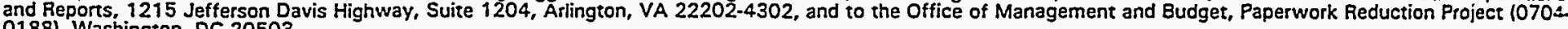
0188 ). Washington. DC 20503.
1.
2. REPORT DATE
February 1996
3. REPORT TYPE AND DATES COVERED
Subcontract Report

4. TITLE AND SUBTITLE

Experimental Investigation of Aerodynamic Devices for Wind Turbine Rotational Speed Control: Phase II

6. AUTHOR(S)

L. Scott Miller, Ph.D.

5. FUNDING NUMBERS

C: $\quad X F-1-11009-3$

TA: WE6 14430

7. PERFORMING ORGANIZATION NAME(S) AND ADDRESS(ES)

L. Scott Miller, Ph.D.

8. PERFORMING ORGANIZATION REPORT NUMBER

Wichita State University

National Institute for Aviation Research

Wichita, Kansas 67260-0044

(316) $689-3410$

9. SPONSORING/MONITORING AGENCY NAME(S) AND ADDRESS(ES)

National Renewable Energy Laboratory

1617 Cole Blvd.

Golden, CO 80401-3393

10. SPONSORINGIMONITORING AGENCY REPORT NUMBER

TP:441-20507

DE96000540

11. SUPPLEMENTARY NOTES

NREL Technical Monitor: Paul Migliore

12a. DISTRIBUTION/AVAILABILITY STATEMENT

National Technical Information Service

U.S. Department of Commerce

5285 Port Royal Road

Springfield, VA 22161

UC-1213

13. ABSTRACT (Maximum 200 words)

In light of the need to produce energy using more robust, reliable and efficient wind turbine designs, a range of new technologies has been explored. Trailing-edge aerodynamic devices represent one such technology that offers the potential for improved horizontal axis wind turbine performance. Indeed, these devices are particularly attractive for two reasons: to prevent turbine rotation speeds from exceeding reasonable limits during high wind or loss of load situations, and for power modulation.

Results from a previous investigation (Miller, 1995) identified and evaluated the capabilities of five trailing-edge aerodynamic devices on a NACA $64_{3}-618$ airfoil. The primary focus of the Phase I work was to enhance braking capabilities for wind turbines during overspeed situations. Power modulation aspects were not specifically addressed.

The aerodynamic capability of each Phase I device configuration was evaluated during low speed wind tunnel tests at Wichita Stzie University (WSU).

Phase 2 efforts, involving additional wind tunnel testing, were performed in an attempt to improve or optimize this particular configuration's performance and applicability for use on wind turbines. The impact of lip length, vent angle, pivot location and device chord were examined further. A significant amount of experimental data was gathered on lift, drag, quarter chord moment, and device hinge moment coefficients as a function of angle of attack and device deflection. This report documents the results of the Phase 2 investigation.

14. SUBJECT TERMS

wind turbine design

15. NUMBER OF PAGES

16. PRICE CODE

17. SECURITY CLASSIFICATION OF REPORT Unclassified
18. SECURITY CLASSIFICATION OF THIS PAGE Unclassified
19. SECURITY CLASSIFICATION OF ABSTRACT Unclassified
20. LIMITATION OF ABSTRACT

UL 